\title{
Numerical Investigations of Pathological Phonation Resulting From a Unilateral Sessile Polyp
}

\author{
by
}

Raymond Greiss, B.Eng.

\begin{abstract}
A thesis submitted to the
Faculty of Graduate and Postdoctoral Affairs

in partial fulfillment of the requirements for the degree of
\end{abstract}

\author{
Master of Applied Science \\ in \\ Mechanical Engineering
}
Ottawa-Carleton Institute for Mechanical and Aerospace Engineering Department of Mechanical Engineering Carleton University
Ottawa, Ontario
December, 2015
CCopyright
Raymond Greiss, 2015




\section{Abstract}

The voice, generated via the modulation of airflow by the vocal folds in the larynx, is studied computationally to improve treatment of voice disorders. A model of a vocal fold with a polyp is developed, and implemented in-house. Structural vibration of the model is computed in the time and frequency domain with the finite element method. Aerodynamic loading is computed with the Bernoulli equation, which is corrected for viscous and unsteady effects. A polyp's mass is the most influential parameter, followed by stiffness and position. Natural frequencies are decreased by increasing mass, and increased by increasing stiffness. Damping due to the polyp's mass is mitigated near fixed walls. Polyp mass distribution is most influential on vibration when spread into the glottis. Pitch-intensity dependence decreases with increasing polyp mass. The polyp disrupts vibration of the vocal fold pair by decreasing the cross-sectional area of the flow, and vibrating out of phase. 
To mom. Thank you for teaching me how to write. 


\section{Acknowledgments}

My first steps into the world of scholarly research would not have been so rewarding if it were not for the immense support offered to me by my supervisors, Professor Joana Rocha and Professor Edgar Matida. Without their dependable technical and financial support, this work would not have been possible. Most importantly, they fostered an open environment where there is no fear of asking a stupid question. To you both, I am exceedingly grateful.

Despite my fondness for numerical computing, I've learned the phrase, "Too much of anything can kill you," to ring true. My thanks go out to Colin, Zahra, Sarah, and Jared for the numerous distracting, yet stimulating, coffee breaks which they have accompanied me on. Their willingness to allow me to bounce ideas off of them have led to the refinement of many aspects of this thesis.

I would not be in a position to pursue graduate studies without the backing of my family, who have always enthusiastically encouraged me to pursue my interests, whether academic or not. Thank you for your persistent love, care, and confidence. I would not have been able to overcome my confusion over turbulence, nor inner turbulence, without your inspiration. 


\section{Table of Contents}

Abstract ii

Acknowledgments iv

$\begin{array}{ll}\text { Table of Contents } & \mathbf{v}\end{array}$

List of Tables $\quad$ ix

List of Figures $\quad$ xi

$\begin{array}{ll}\text { Nomenclature } & \text { XV }\end{array}$

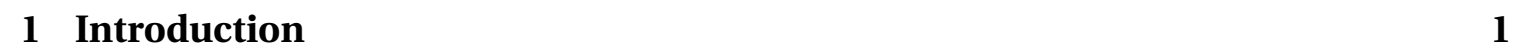

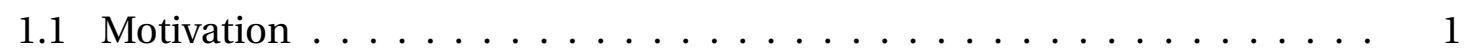

1.2 Fundamentals of Phonation $\ldots \ldots \ldots \ldots \ldots$

$1.2 .1 \quad$ Aerodynamic transfer of energy $\ldots \ldots \ldots \ldots$

1.2 .2 Structural properties of the vocal folds $\ldots \ldots \ldots$. . . . . . 6

1.2 .3 Body-cover theory of phonation $\ldots \ldots \ldots \ldots$

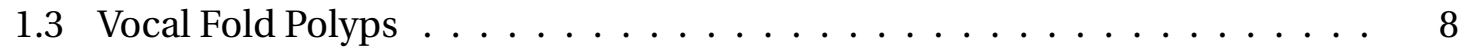

1.4 State of the Art . . . . . . . . . . . . . . . . 9

1.4.1 Computational vocal fold modeling . . . . . . . . . . . . . 9

1.4 .2 Computational models of polyps . . . . . . . . . . . . . 11 
1.5 Objectives of the thesis $\ldots \ldots \ldots \ldots \ldots \ldots \ldots$

1.6 Structure of the Thesis $\ldots \ldots \ldots \ldots \ldots \ldots$. . . . . . . . . . . . . . 14

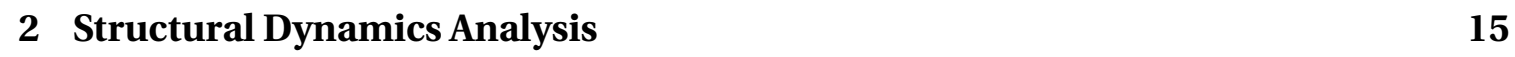

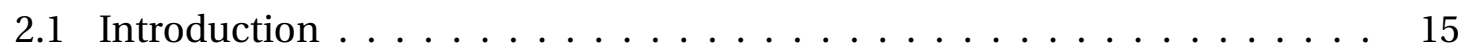

2.2 Vocal Fold Model $\ldots \ldots \ldots \ldots \ldots$

2.2 .1 Geometry . . . . . . . . . . . . . . . . . . 15

2.2 .2 Material behaviour . . . . . . . . . . . . . . . 18

2.3 Pathological Modeling . . . . . . . . . . . . . . . . . . . . . 20

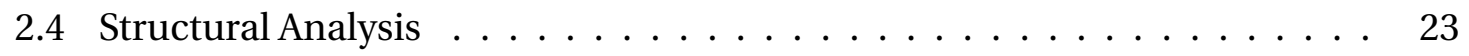

2.4 .1 Governing equations $\ldots \ldots \ldots \ldots \ldots$. . . . . . . . . . 24

2.4 .2 FEM formulation $\ldots \ldots \ldots \ldots \ldots$

2.4 .3 Deriving the four-node tetrahedral shape functions $\ldots . \ldots . . .29$

2.4 .4 Deriving the element mass matrix $\ldots \ldots \ldots$. . . . . . . . . 33

2.5 Solution Methods . . . . . . . . . . . . . . . . . . . . . . . 34

2.5 .1 Direct integration of the equation of motion $\ldots \ldots \ldots$. . . . . . . 34

2.5 .2 Solution of the Eigenproblem $\ldots \ldots \ldots$. . . . . . . . . 37

2.5 .3 Boundary and initial conditions $\ldots \ldots \ldots \ldots$

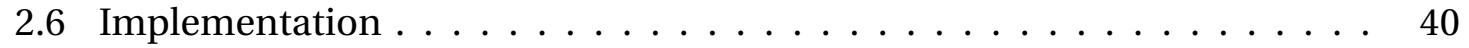

$2.7 \quad$ Verification and Validation $\ldots \ldots \ldots \ldots \ldots \ldots \ldots$

$2.7 .1 \quad$ Free vibration of a three-dimensional vocal fold $\ldots . . . . . . . . .44$

2.7 .2 Forced vibration of a cantilever beam . . . . . . . . . . . . 46

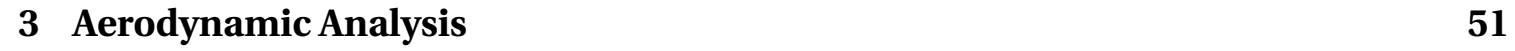

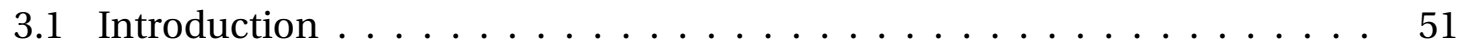

3.2 Justification for Bernoulli Flow $\ldots \ldots \ldots \ldots \ldots$. . . . . . . . . 51

$3.3 \quad$ Bernoulli Flow Methodology $\ldots \ldots \ldots \ldots$ 
3.3 .1 Computing the pressure distribution $\ldots \ldots \ldots \ldots$

3.3.2 Computing the volume flow rate $\ldots \ldots \ldots \ldots 5$

3.3 .3 Computing the point of flow separation $\ldots \ldots \ldots$. . . . . . . . . 56

3.3 .4 Accounting for energy losses . . . . . . . . . . . . . . 57

3.4 Computation of Aerodynamic Forces $\ldots \ldots \ldots \ldots$

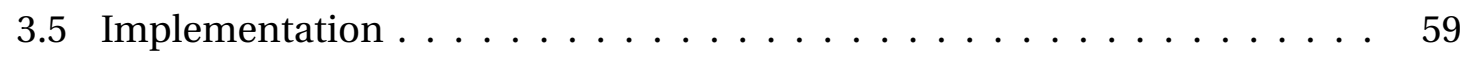

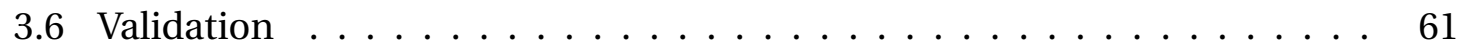

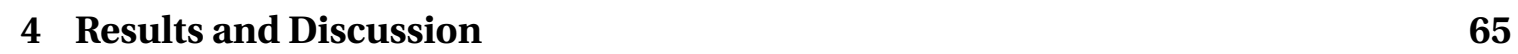

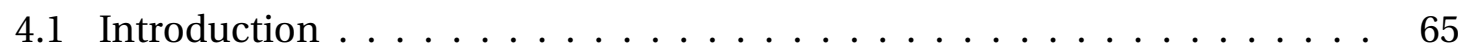

4.2 Sensitivity Analysis of Polyp Parameters . . . . . . . . . . . . . . . . 66

4.2 .1 Spatial convergence analysis . . . . . . . . . . . . . 66

4.2 .2 Interaction of mass and stiffness $\ldots \ldots \ldots$. . . . . . . . 68

$4.2 .3 \quad$ Interaction of mass and position $\ldots \ldots \ldots \ldots \ldots$. . . . . 71

4.2 .4 Effects of aspect ratio changes . . . . . . . . . . . . . 74

4.3 Influence of Polyps on the Pitch-Intensity Dependence $\ldots . . . . . . .78$

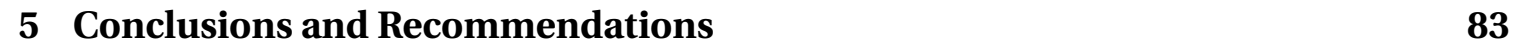

5.1 Summary and Contributions $\ldots \ldots \ldots$. . . . . . . . . . 83

5.2 Conclusions $\ldots \ldots \ldots \ldots \ldots \ldots \ldots$

5.3 Recommendations for Future Work $\ldots \ldots \ldots \ldots$

\begin{tabular}{lr}
\hline List of References & 88
\end{tabular}

\begin{tabular}{|lll}
\hline Appendix A Derivation of the Linear Triangular Element Matrices & 95
\end{tabular}

A.1 Derivation of the Shape Functions . . . . . . . . . . . . . . . . 95

A.2 Deriving the Element Mass Matrix and Stiffness Matrices . . . . . . . . 98 
\begin{tabular}{|ll}
\hline Appendix B Contour and Relative Influence Plots & 100
\end{tabular}

B.1 Interaction of mass and stiffness $\ldots \ldots \ldots \ldots$. . . . . . . . . . . 100

B.2 Interaction of mass and position $\ldots \ldots \ldots \ldots \ldots$ 


\section{List of Tables}

2.1 Physical parameters for the three-layer transversely isotropic vocal fold |3] 19

2.2 Unilateral sessile polyp model material properties . . . . . . . . . . . . 23

$2.3 \quad$ Unilateral sessile polyp model parameters . . . . . . . . . . . . . . . 23

2.4 Comparison of computed natural frequencies of the transversely isotropic vocal fold for the mesh with 7314 degrees of freedom . . . . . . . . . . . . 45

2.5 Numerical properties of the loaded cantilever beam . . . . . . . . . . . . 48

2.6 Comparison of computed natural frequencies with exact solution for the cantilever beam . . . . . . . . . . . . . . . . . . . 49

$3.1 \quad$ Flow model properties $\ldots \ldots \ldots \ldots$. . . . . . . . . . . . . . . 61

$4.1 \quad$ Polyp parameters and nominal state values . . . . . . . . . . . . . . . 66

4.2 Taguchi L16 OA for polyp parameter states . . . . . . . . . . . . . . . . . 67

$4.3 \quad$ Mass and stiffness interaction simulation set-up, and first four computed natural frequencies $\ldots \ldots \ldots \ldots \ldots \ldots$. . . . . . . . . . . . . . . . .

$4.4 \quad$ Mass and position interaction simulation set-up, and first four computed natural frequencies $\ldots \ldots \ldots \ldots \ldots \ldots \ldots$

$4.5 \quad A: B$ aspect ratio simulation set-up, and first four computed natural fre-

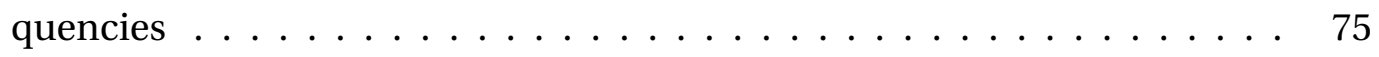

$4.6 \quad A: C$ aspect ratio simulation set-up, and first four computed natural fre-

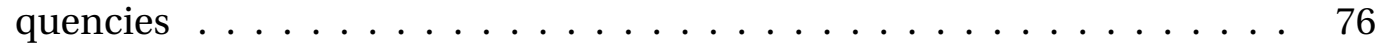


$4.7 \quad B: C$ aspect ratio simulation set-up, and first four computed natural fre-

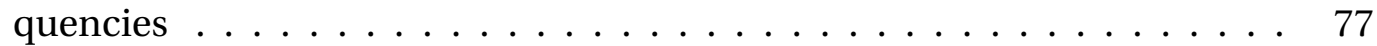




\section{List of Figures}

$1.1 \quad$ (a) Midcoronal plane schematic of the larynx. (b) Schematic of the periodic mucosal wave propagation on the surface of the vocal folds, from |17 modified]. (c) Schematic of the top view of the vocal folds from [19|. . . . . 4

1.2 A representation of the (a) abducting, convergent, and (b) adducting, divergent profiles of the vocal folds during mucosal wave propagation. . . . 5

1.3 Body-cover model of a single vocal fold. . . . . . . . . . . . . . . . . 8

$2.1 \quad$ Vocal fold geometry based on CT data $|4|] \ldots \ldots \ldots \ldots$

2.2 Ellipsoid hemisphere model of a polyp, and the minor axes parameters. . $\quad 20$

2.3 Range of polyp position parameter, $c . \ldots \ldots \ldots \ldots \ldots$. . . . . . . . 22

2.4 One-dimensional mass-spring-damper system, and its dynamic equilibrium force diagram analogue. . . . . . . . . . . . . . . . . . . 24

2.5 Hamilton's principle, or the principle of least action. Scalar energy functionals are used to distinguish the correct path of the particle between $u_{1}$

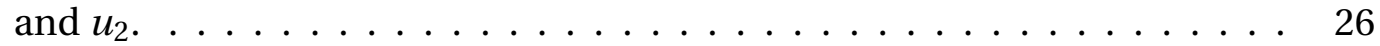

2.6 Three-dimensional vocal fold is discretized using tetrahedral elements. Values of $\mathbf{q}$ are stored at node points, and other values within the field are interpolated using element specific shape functions. . . . . . . . . . . . 28

2.7 Elements incorporated in the vocal fold models. . . . . . . . . . . . . . . . . 29

2.8 Sub-tetrahedrals defined by an arbitrary point within the element. . . . . . 30 
2.9 Fixed planes on the vocal fold. Nodes on the anterior and posterior planes, and the lateral surface are fixed. . . . . . . . . . . . . . . . . . . . 39

2.10 The contact line is set at $y_{c}=0.08876 \mathrm{~mm}$ from the medial surface of the

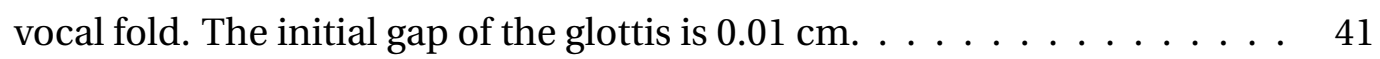

2.11 Structural solver software flowchart. . . . . . . . . . . . . . . . . . . 43

2.12 Convergence of the transversely isotropic vocal fold model. . . . . . . . . . 44

2.13 (a) Mode 1. Left: Computed solution. Right: Solution from literature $|4| . . \quad$. 45

2.14 Geometry of the loaded cantilever beam $\ldots \ldots$. . . . . . . . . . . 48

2.15 Spatial convergence of the cantilever beam. . . . . . . . . . . . . . 49

2.16 Temporal convergence of the cantilever beam. . . . . . . . . . . . . 50

3.1 Intraglottal pressure is solved along the vocal fold surface in the downstream direction, knowing that subglottal pressure is constant in time. . . 55

3.2 Streamline between the lungs and the plane of separation between the vocalfolds $\ldots \ldots \ldots \ldots \ldots \ldots \ldots \ldots$

3.3 Aerodynamic forces are computed at the centroid of each "wet" segment, and split into component forces. . . . . . . . . . . . . . . . . . . 59

3.4 Component forces are distributed to the nodes of the mesh via node by node lumping. . . . . . . . . . . . . . . . . . . . . 60

3.5 Comparison of pressure distribution along separate vocal fold models. Blue: Decker \& Thomson, 2007 |62|. Red: Vocal fold incorporated in this study . . . . . . . . . . . . . . . . . . . . . . . 62

3.6 Computed flow rate for 200 Pa subglottal pressure. . . . . . . . . . . . . . 62

3.7 Snapshot of the vocal folds at abduction and adduction. . . . . . . . . . . 63

3.8 Time-history of displacement of a point on the vocal fold surface near the top edge of the medial plane. . . . . . . . . . . . . . . . . 64 
$4.1 \quad$ Simulation set-up for examination of the interaction of mass and stiffness. The mass and stiffness in the three-dimensional mesh is varied for multiple cases. . . . . . . . . . . . . . . . . . . 68

4.2 Contour plot of fundamental frequency as a function of mass and stiffness. 70

4.3 Relative influence of mass and stiffness on fundamental frequency. Left:

$\omega_{1}$ as a function of mass for $E_{1}$. Right: $\omega_{1}$ as a function of stiffness for $M_{1}$.

4.4 Simulation set-up for examination of the interaction of mass and position.

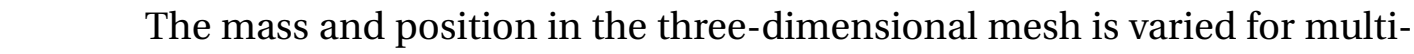

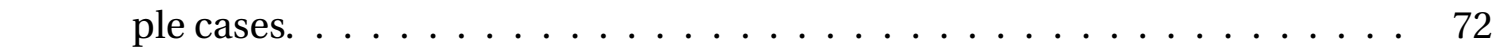

4.5 Contour plot of fundamental frequency as a function of mass and position. 73

4.6 Relative influence of mass and position on fundamental frequency. Left:

$\omega_{1}$ as a function of mass for $E_{1}$. Right: $\omega_{1}$ as a function of stiffness for $M_{1}$. 74

$4.7 \quad$ Variation in natural frequencies as a function of $A: B . \ldots \ldots$

4.8 Variation in natural frequencies as a function of $A: C$. $\ldots \ldots \ldots$

4.9 Variation in natural frequencies as a function of $B: C$. . . . . . . 78

4.10 Set-up for simulations of forced vibration. Both sets of vocal folds are sim-

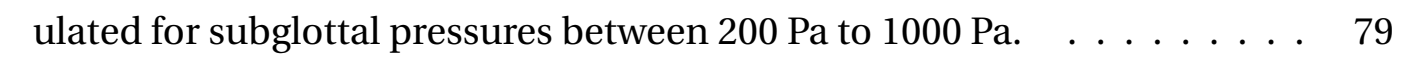

4.11 Pitch-intensity curves for the vocal fold pairing with a polyp of mass $M_{1}$. . 80

4.12 Pitch-intensity curves for the vocal fold pairing with a polyp of mass $M_{2}$. . 81

A.1 Sub-triangles defined by an arbitrary point within the element. . . . . . . . 96

B.1 Contour plot of second natural frequency as a function of mass and stiffness. 101

B.2 Relative influence of mass and stiffness on second natural frequency. . . . 101

B.3 Contour plot of third natural frequency as a function of mass and stiffness. 102

B.4 Relative influence of mass and stiffness on third natural frequency. . . . . 102

B.5 Contour plot of fourth natural frequency as a function of mass and stiffness. 103

B.6 Relative influence of mass and stiffness on fourth natural frequency. . . . 103 
B.7 Contour plot of second natural frequency as a function of mass and position. 104

B.8 Relative influence of mass and position on second natural frequency. . . . 104

B.9 Contour plot of third natural frequency as a function of mass and position. 105

B.10 Relative influence of mass and position on third natural frequency. . . . . 105

B.11 Contour plot of fourth natural frequency as a function of mass and position.106

B.12 Relative influence of mass and position on fourth natural frequency. . . . 106 


\title{
Nomenclature
}

\author{
$A, B, C$ \\ Minor axes radii of polyp elliposid in $z, x$, and $y$, respectively [m] \\ $A_{i}$ \\ Cross-sectional flow area at node $i\left[\mathrm{~m}^{2}\right]$ \\ $[B]$ \\ Strain matrix \\ $[C]$ \\ Global damping matrix $[\mathrm{kg} / \mathrm{s}]$ \\ $c$ \\ Polyp's center coordinate [m] \\ $[D]$ \\ Stiffness matrix $[\mathrm{Pa}]$ \\ $E_{i}$ \\ Young's modulus in the $i$ direction [Pa] \\ $E_{L}$ \\ Young's modulus of vocal fold ligament [Pa] \\ $E_{P}$ \\ Young's modulus of vocal fold polyp [Pa] \\ $[\hat{F}]$ \\ Effective applied force vector $[\mathrm{N}]$ \\ $[F]$ \\ Applied force vector $[\mathrm{N}]$ \\ $\mathbf{F}_{i}$ \\ Applied force in direction $i[\mathrm{~N}]$ \\ $G_{i j}$ \\ Shear modulus between axes $i$ and $j[\mathrm{~Pa}]$ \\ $I$ \\ Second moment of area $\left[\mathrm{m}^{4}\right]$
}




\begin{tabular}{|c|c|}
\hline$[K]$ & Global stiffness matrix $\left[\mathrm{kg} / \mathrm{s}^{2}\right]$ \\
\hline$[\hat{K}]$ & Effective global stiffness matrix $\left[\mathrm{kg} / \mathrm{s}^{2}\right]$ \\
\hline$\left[k_{e}\right]$ & Element stiffness matrix $\left[\mathrm{kg} / \mathrm{s}^{2}\right]$ \\
\hline$L$ & Length of cantilever beam [m] \\
\hline$L_{i}$ & Volume coordinate $i$ \\
\hline$[M]$ & Global mass matrix $[\mathrm{kg}]$ \\
\hline$M$ & Mach number \\
\hline$\left[m_{e}\right]$ & Element mass matrix $[\mathrm{kg}]$ \\
\hline$[N]$ & Shape function matrix \\
\hline$P$ & Static pressure $[\mathrm{Pa}]$ \\
\hline$P_{L}$ & Lung static pressure $[\mathrm{Pa}]$ \\
\hline$P_{S}$ & Static pressure at flow separation $[\mathrm{Pa}]$ \\
\hline$P_{\text {subglottal }}$ & Subglottal static pressure $[\mathrm{Pa}]$ \\
\hline$P_{\text {supraglottal }}$ & Supraglottal static pressure $[\mathrm{Pa}]$ \\
\hline$\dot{Q}$ & Volume flow rate $\left[\mathrm{m}^{3} / \mathrm{s}\right]$ \\
\hline $\mathbf{q}$ & Displacement vector $[\mathrm{m}]$ \\
\hline$\dot{\mathbf{q}}$ & Velocity vector $[\mathrm{m} / \mathrm{s}]$ \\
\hline$\ddot{\mathbf{q}}$ & Acceleration vector $\left[\mathrm{m} / \mathrm{s}^{2}\right]$ \\
\hline
\end{tabular}




\begin{tabular}{|c|c|}
\hline$T$ & Kinetic energy functional $[\mathrm{J}]$ \\
\hline$T_{i}$ & Period corresponding with $i$ th natural frequency $[\mathrm{s}]$ \\
\hline$t$ & Time $[s]$ \\
\hline$t_{0}$ & Time constant $[\mathrm{s}]$ \\
\hline$U$ & Potential (strain) energy functional $[\mathrm{J}]$ \\
\hline$u_{i}, v_{i}, w_{i}$ & Displacements at node $i$ in $x, y$, and $z$ [m] \\
\hline$V$ & Volume $\left[\mathrm{m}^{3}\right]$ \\
\hline$V_{i}$ & Volume of sub-tetrahedral $i\left[\mathrm{~m}^{3}\right]$ \\
\hline$\vec{v}$ & Particle velocity $[\mathrm{m} / \mathrm{s}]$ \\
\hline$W_{n c}$ & Non-conservative energy [J] \\
\hline$x, y, z$ & Cartesian coordinates $[\mathrm{m}]$ \\
\hline$x_{i}, y_{i}, z_{i}$ & Coordinates of node $i[\mathrm{~m}]$ \\
\hline$y_{C}(x), y_{L}(x), y_{B}(x)$ & Cover, ligament, and body surface definition curves $[\mathrm{m}]$ \\
\hline$y_{c}$ & Coordinate of contact line $[\mathrm{m}]$ \\
\hline$\alpha$ & Empirical vena contracta energy loss factor \\
\hline$\alpha_{P}$ & Polyp stiffness factor \\
\hline$\beta$ & Newmark integration parameter \\
\hline & Newmark integration parameter \\
\hline
\end{tabular}


$\gamma_{S}$

$\Delta t$

$\delta$

$\epsilon$

$\lambda$

$\mu$

$v_{i j}$

$\rho$

$v_{P}$

$\rho_{P}$

$\sigma$

$\phi$

$\omega_{i}$
Empirical separation area ratio

Time step [s]

Virtual displacement [m]

Strain vector

Generalized eigenvalue

Dynamic viscosity [Pa s]

Poisson's ratio in $j$ from extension in $i$

Density $\left[\mathrm{kg} / \mathrm{m}^{3}\right]$

Poisson's ratio of vocal fold polyp

Polyp density $\left[\mathrm{kg} / \mathrm{m}^{3}\right]$

Stress vector $[\mathrm{Pa}]$

Mode shape [m]

$i$ th natural frequency $[\mathrm{Hz}]$ 


\section{Chapter 1}

\section{Introduction}

The voice plays an incredible role in human life. Aside from providing a general indication of health [1, p. 171], the voice plays a fundamental role in expressing individual identity, and has impacts on numerous facets of life, such as speech and music. Consequently, its loss has major psychological impact.

Voice disorders have the power to detriment, or fully eliminate the voice. The study of voice disorders and their treatment are thus carried out with a view to protecting this precious resource. Computational studies of the underlying physics of the voice $[2-4]$,

effects of disease [5-7], and surgical intervention [8] on speech (phonation) have been carried out as an effort to improve our understanding of the effects of voice disorders, their pathogenesis, and treatment options [9]. In that vein, this thesis documents the development of a physiologically accurate numerical model of a vocal fold polyp as a preliminary step in quantifying its effects on phonation.

\subsection{Motivation}

Voice disorders may affect anyone, and lifetime incidence rates are common at $28.8 \%$. In professions which rely on heavy use of the voice, such as teachers, voice disorders 
are even more prevalent, with lifetime incidence rates reported as high as $57.7 \%$ [10]. Breakthroughs in the treatment of voice disorders require an in-depth understanding of the biomechanics which govern phonation. Although advances in the understanding of the basics of phonation have been made since the 1940's, there are yet many questions to be answered regarding the effects of pathologies [11]. Historically, research into the effects of pathological tissues have led to advances in vocal fold prosthetic development [12, 13], and refinement of surgical techniques [8, 14]. Despite these advances, the surgical treatment of some pathologies, such as vocal fold polyps, may still lead to a further detriment in voice quality $[9]$.

The most common pathology of the vocal folds are polyps [15, p. 121], which are lesions that form on the vocal fold surface [16]. Patients who present with vocal fold polyps are advised to undergo a surgical procedure for their removal, followed by speech therapy to mitigate any resulting biomechanical disruption [15, p. 122]. Analysis of the effects of polyps from a mechanical engineering perspective is ideal, as the underlying physics involves a combination of aerodynamics and structural dynamics.

The present work develops a parameterized finite element model of vocal fold polyps, which is then used to assess the changes in phonation properties based on various qualities of the vocal fold polyp. This model provides important information regarding the disruptive effects of polyp shape, position, mass, and stiffness on vocal fold vibration, and phonation.

\subsection{Fundamentals of Phonation}

Phonation is the result of the conversion of aerodynamic power into acoustic power, which is heard as the voice [17, p. 699]. A simplified representation of the relevant anatomy is illustrated in Fig.1.1(a). A steady stream of air from the lungs passes through 
the larynx, which houses the vocal folds. The vocal folds, under the influence of the applied aerodynamic forces, begin to vibrate, effectively modulating the airflow by periodically obstructing the flow of air [1, p. 101]. The vocal folds oscillate, abducting (opening) and adducting (closing), which varies the area of the void between the vocal folds, called the glottis $[18$. This oscillatory motion on the surface of the vocal folds is termed mucosal wave propagation [17, pp. 702-703], and the frequency of this motion is the fundamental frequency of vibration of the system. A schematic of a cycle of mucosal wave propagation is illustrated in Fig. $1.1(\mathrm{c})$

The subsections which follow will outline the physics which governs the selfoscillation of the vocal folds. It is important to understand these concepts at a fundamental level such that relevant physical parameters can be identified for accurate modeling. Aerodynamic to structural power conversion will be considered first, followed by a description of the vocal folds' structural properties.

\subsubsection{Aerodynamic transfer of energy}

The key to the self-oscillation of the vocal folds is temporal asymmetry of the average intraglottal pressure. Average intraglottal pressure must be greater during abduction than during adduction to allow a positive net amount of energy to be transferred from the airstream to the vocal folds $|20|$. This mechanism was first postulated by Titze in 1988 [21], and confirmed through experimentation and simulation by Thomson in $2005[20]$. To illustrate, Fig. 1.2(a) and Fig. 1.2(b) show the abduction and adduction phases, respectively, during oscillation. Static pressure upstream of the vocal folds is denoted as subglottal pressure, $P_{\text {subglottal }}$, and pressure downstream from the vocal folds is denoted as supraglottal pressure, $P_{\text {supraglottal }}$. These pressures are assumed to remain constant during phonation. Supraglottal pressure is equal to ambient pressure 


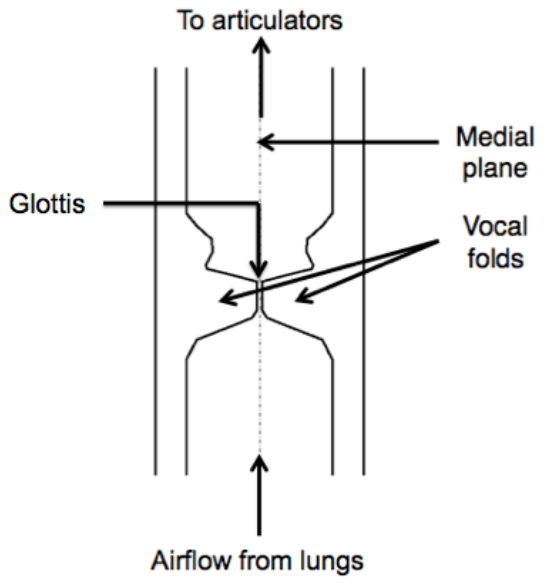

(a)

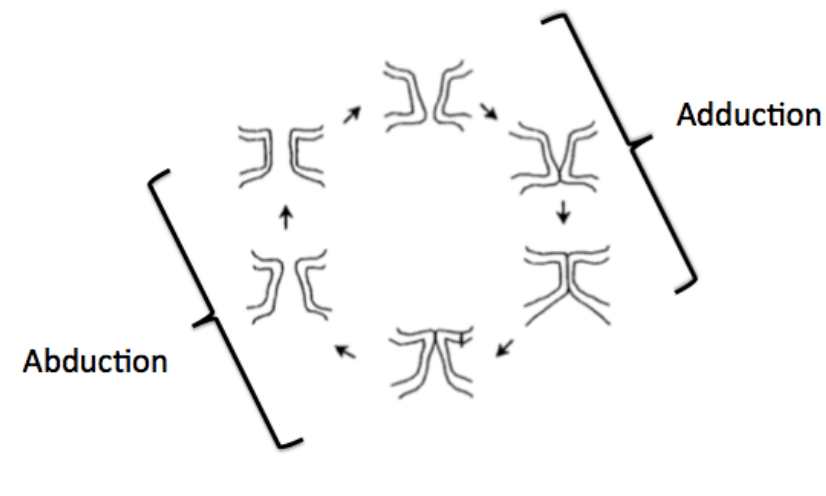

(b)

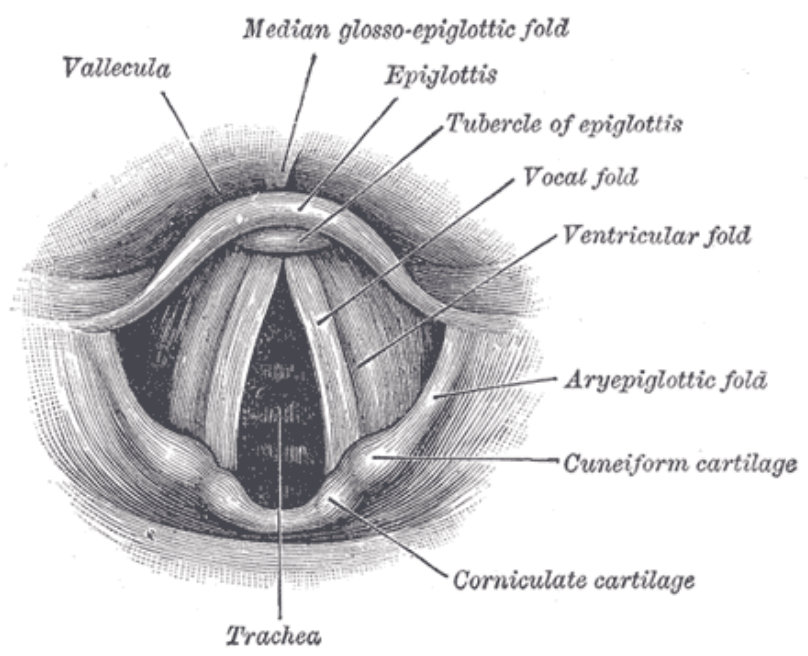

(c)

Figure 1.1: (a) Midcoronal plane schematic of the larynx. (b) Schematic of the periodic mucosal wave propagation on the surface of the vocal folds, from [17, modified]. (c) Schematic of the top view of the vocal folds from |19|.

conditions, and subglottal pressure is larger than supraglottal pressure during phonation. In Fig. $1.2(\mathrm{a})$ and Fig. $1.2(\mathrm{~b})$, we assume $P_{\text {supraglottal }} \approx 0$ to ignore any aerodynamic and acoustic loading effects to simplify the example.

In the abduction phase, the vocal folds are in a convergent profile, as in Fig. 1.2(a), and subglottal pressure forces the vocal folds apart. In this orientation, viscous action of 


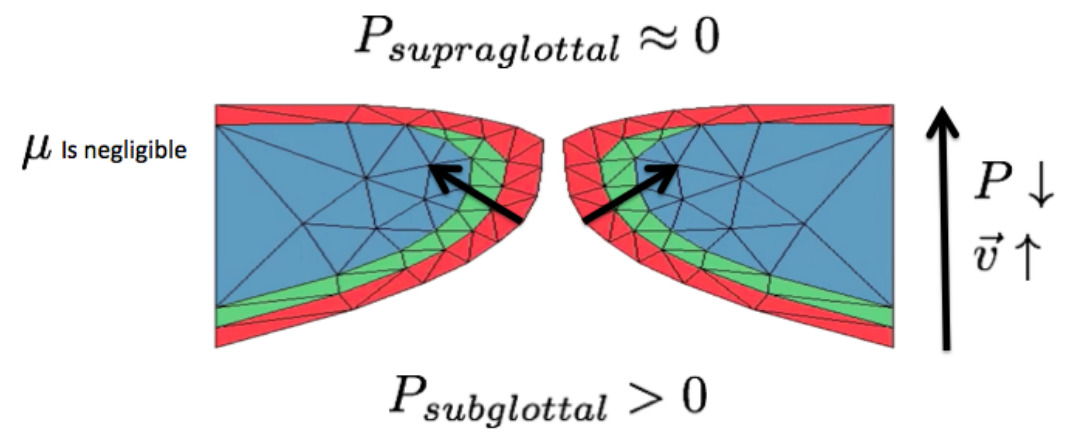

(a)

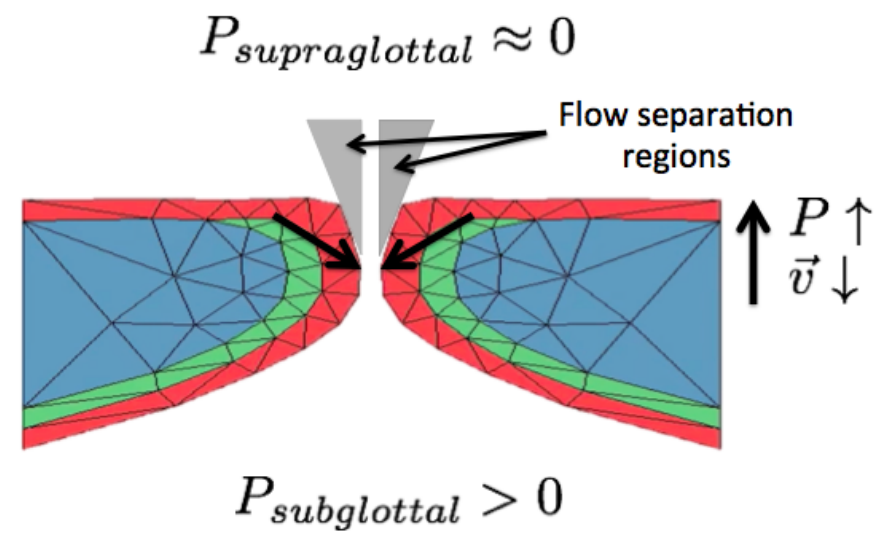

(b)

Figure 1.2: A representation of the (a) abducting, convergent, and (b) adducting, divergent profiles of the vocal folds during mucosal wave propagation.

the air, represented by dynamic viscosity, $\mu$, is negligible, due to the separation between the inviscid core, and the viscous regions at the vocal fold walls. In other words, the air can be considered inviscid [20]. Under inviscid conditions, and assuming incompressible flow (due to $M<0.3$ ), where density, $\rho$, is assumed to be constant in space and time, Bernoulli's equation may be used to relate static pressure and air velocity. Bernoulli's equation states

$$
P+\frac{1}{2} \rho \vec{v}^{2}=\text { constant }
$$

where $P$ is static pressure, and $\vec{v}$ is air velocity. This is physically interpreted as meaning 
that as velocity increases, pressure decreases, and vice-versa [22, p. 183]. Thus, as the cross-sectional area of the glottis decreases, air velocity increases to conserve mass, and consequently, static pressure decreases.

In the adduction phase, the vocal folds are in a divergent profile, as in Fig.1.2(b). Air velocity decreases in the downstream direction, and according to Bernoulli's equation, pressure increases. In the stream wise direction, for the divergent profile, pressure must increase to zero, whereas in the convergent profile, pressure decreases to zero. This establishes the driving pressure asymmetry: the average intraglottal pressure is greater in the convergent phase than in the divergent phase, which represents a net transfer of energy from the airstream to the vocal fold tissue [21].

Of note, during the adduction phase, the influence of viscous action increases as the vocal fold surfaces come together [20]. A combination of viscous shear stress on the air, as well as an adverse pressure gradient forming in the stream wise direction [22, p. 716] causes flow separation on the vocal fold surface. This has the effect of decreasing the area of the inviscid core of the flow, as illustrated in Fig. 1.2(b), This creates a pulsatile glottal jet, which is a major contributor of the generated sound [11].

\subsubsection{Structural properties of the vocal folds}

Various qualities of the voice may be directly related to the oscillatory mucosal wave propagation on the vocal fold surface. This is somewhat unintuitive, as the major source of sound generation related to the voice is due to the pulsatile glottal jet [11], which is an aerodynamic property. However, experimental modal analysis of the vocal folds have shown that natural frequencies of vibration do not vary significantly from phonation frequencies [2]. Furthermore, the fundamental frequency of mucosal wave propagation is directly related to the lowest frequency harmonic of the voice's spectrum [18]. Hence, the study of the structural vibration of the vocal folds offers considerable insight into the 
production of the voice.

This line of thought was postulated as early as the 1950's by van den Berg [23], who developed the mathematical framework of myoelastic-aerodynamic theory of phonation. This theory postulates that acoustic parameters such as pitch and intensity are governed by the mass and viscoelastic properties of the vocal folds [17, p. 700]. This theory may be used to relate several acoustic properties to structural vibration directly. For example, pitch increases as the length of abduction increases. This has the effect of reducing the mass per unit length of vocal fold exposed to the aerodynamic forces. Increasing tension in the vocal folds also increases the pitch. Intensity of the voice increases as the period of the adduction phase of mucosal wave propagation increases $\mid 1$, pp. 159-160]. The myoelastic-aerodynamic framework is the currently accepted theory for the governing physics behind vocal fold self-oscillation [24].

\subsubsection{Body-cover theory of phonation}

To relate properties of the voice to structural vibration, myoelastic-aerodynamic theory requires a quantitative definition of the vocal folds' material properties and structure. Body-cover theory, developed by Hirano in 1974 [25], defines the vocal folds as being composed of two materials, the body and the cover. The orientation of these materials on the vocal fold is illustrated in Fig. 1.3. Each of these layers has its own distinctive set of material properties. The body is the more stiff portion of the vocal fold, which is composed of fibrous tissue, such as muscle and ligament. The cover is the less stiff material, called the mucosal layer, or the lamina propria [26], which displays the wavelike motion associated with the mucosal wave [27, p. 8]. 


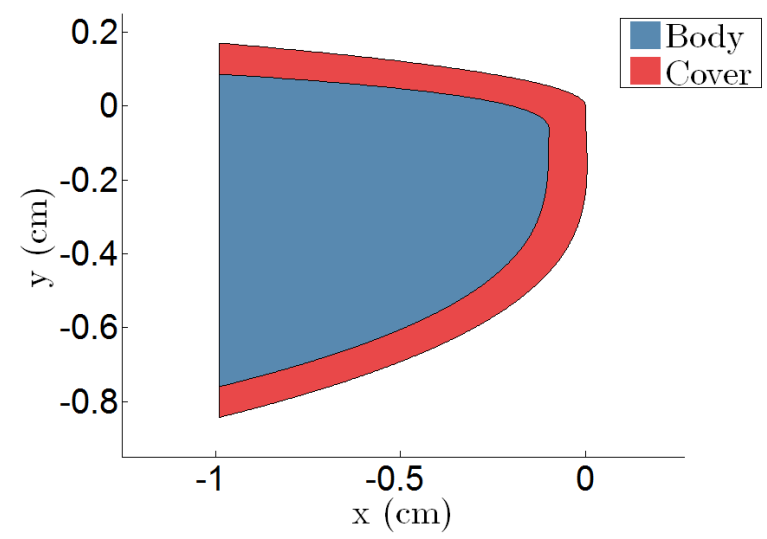

Figure 1.3: Body-cover model of a single vocal fold.

\subsection{Vocal Fold Polyps}

Vocal fold polyps are the most common laryngeal disease, resulting from submucosal hemorrhaging caused by hyperkinetic motion of the vocal folds [15, pp. 121-123]. These benign lesions manifest as medial protrusions on the vocal fold's mucosal surface, and may be unilateral or bilateral (appearing on one, or both vocal folds, respectively) [28]. The added mass and stiffness of a polyp alters the propagation of the mucosal wave across the vocal fold surface, and protrudes into the glottis, subsequently altering the aerodynamics within the larynx [11]. These disruptions result in symptoms of dysphonia (difficulty in producing speech), such as hoarseness [29], vocal fatigue, breathiness, and rough vocal quality [11].

Polyps appear in three forms:

1. Sessile: An exophytic (outward growing) protrusion [27, p. 24] with a balloon-like appearance $|29|$.

2. Pedunculated: A stalk-like protrusion from a single position [29].

3. Polypoid Corditis (Reinke's Edema): The accumulation of mass across the entire vocal fold mucosal surface [27, p. 25]. 
Each form of polyp is hypothesized to vary the characteristic vibration of the vocal folds differently due to their varied distribution of excess mass and stiffness. This research will focus on modeling unilateral sessile polyps.

\subsection{State of the Art}

Computational models of phonation have evolved significantly over the course of several decades. Advancements in the formulation of the equations of motion, aerodynamic modeling, structural and aerodynamic coupling, and geometric models of the vocal folds have led to a marked improvement in the ability to study phonation. The following sections outline some major advancements in the modeling of vocal folds and polyps.

\subsubsection{Computational vocal fold modeling}

Computational studies of vocal fold vibration have been reported since the late 1960's. Early models of vocal fold vibration make use of lumped element methods, which simulate the vocal folds as a system of masses connected by springs and dampers. Viscoelastic properties are applied to the models in the form of spring and damping properties [16]. One of the earliest such models was developed by Ishizaka and Flanagan in $1972[30$. This model approximates the vocal folds as two connected masses, and simulated aerodynamic loading using Bernoulli flow with an assumed position of flow separation. This model illustrates an excellent ability to capture mucosal wave propagation and self-oscillation, and has since been used to study the effects of various pathologies on phonation, such as asymmetries $[8,31-33 \mid$ and polyps $[6]$. Although these models have produced data which is in close agreement with in-vivo studies, lumped element models do not have the spatial resolution to capture fine details of vocal fold vibration. 
Since the development of the two mass model, this issue has been addressed by assuming more masses on the vocal folds. Notably, Titze developed a 16 mass model [34 35]. To the author's understanding, lumped element models have been reported with as many as 175 masses approximating the vocal fold geometry [16].

Researchers have addressed the issue of spatial resolution through the use of finite element models. These models discretize a continuum body into a collection of finite elements, and approximate the solution of the equation of motion for the assembly of these elements. The seminal finite element model of vocal fold vibration was published in 2000 by Alipour, Berry, and Titze |2|. This study modeled the vocal fold in three dimensions using two-dimensional elements in 15 layers along the anterior-posterior direction, with each layer connected to its neighbors by shear string forces. This model approximated the aerodynamic forces using Bernoulli flow, but was subsequently updated by incorporating a finite volume solution of the incompressible Navier-Stokes equations [36]. These models incorporated three distinct materials, the body, cover, and ligament, each of which was characterized by distinct transversely isotropic material properties, and idealized geometries. Models are transversely isotropic due to the presence of fibers which run in the anterior-posterior direction in the vocal folds, which results in a plane of isotropy with distinct properties in the longitudinal direction. Although the models were successful in capturing physiologically realistic vibratory behaviour, they were criticized for their pseudo three-dimensional geometry, and use of Bernoulli flow. To capture the aerodynamics of the glottal jet, a fully three-dimensional model of the vocal folds and the flow field is required [11].

Rosa et al. $|3|$ improved on this work by developing a three-dimensional finite element model of the larynx and vocal folds with a fully-coupled Navier-Stokes solver. Due to the three-dimensional nature of the finite elements, anterior-posterior translations 
could be resolved, as well as the glottal jet in the flow field. The extension of these continuum models into the third dimension has facilitated exploration of the underlying physics of phonation. For instance, coupling in the acoustic domain has been explored by Daily and Thomson [37] by assuming a slightly compressible fluid model to capture acoustic wave propagation. Studies of pathologies, such as vocal fold trauma [38, 39], nodules [7], and asymmetries [40] have also been explored by updating these models. Fundamental work in understanding phonation has also been explored through the use of these models, such as by Gunter [41], who explored the kinematics and collision forces of the vocal folds, and Thomson, Mongeau, and Frankel [20], who explored aerodynamic transfer of energy through temporal asymmetry of average intraglottal pressure.

\subsubsection{Computational models of polyps}

Computational models of vocal fold polyps have demonstrated excellent ability to capture the influence of a polyp's excess mass and stiffness on disrupting mucosal wave propagation. One such study by Zhang and Jiang $[6 \mid$ simulated the polyp as a cubic mass attached to the lower portion of a two-mass non-linear lumped element vocal fold model. Polyp size, stiffness, and damping were varied to assess variation in vocal fold vibration. Very small polyps were not found to vary the periodicity of the vibration, however, polyps larger than a threshold size led to asymmetric vibrations. Decreasing stiffness and damping of the attachment between the polyp and the vocal fold was found to lead to aperiodic vibrations, however, the biomechanical analogue of this result is difficult to infer. In addition, geometric protuberance cannot be studied with such a model, as the lumped element vocal fold model does not have the spatial resolution to precisely capture aerodynamic behaviour.

To improve spatial resolution, incorporation of polyps with existing finite element 
models of vocal folds have been used. Jiang, Diaz, and Hanson [5] simulated a polyp as a $2 \times 2 \times 2 \mathrm{~mm}^{3}$ mass on the midpoint of the lateral surface of a finite element model of a vocal fold. The polyp was treated as an isotropic material, with five times the Young's Modulus of the body layer of the vocal fold. The addition of a polyp was shown to decrease the frequencies of vibration, and significantly alter the modes of vibration. Notably, this model was developed in 1998, prior to the publication of the seminal finite element model of Alipour, Berry, and Titze |2| in 2000. To the author's knowledge, no idealized geometric model of a vocal fold was yet available, nor accepted within the literature. Despite this, no indication is given regarding how the geometry was developed, nor was the justification of isotropy made.

Deguchi and Kawahara [7] performed a similar study of the addition of a nodule on a finite element vocal fold model. Though the authors consider the additional mass a "nodule", this unilateral mass was situated on the surface of the vocal fold, which fits the clinical definition of a polyp. By definition, nodules usually present as bilateral lesions $[28 \mid$. Practically, however, some authors consider the two pathologies to be the same, despite whether they present as unilateral or bilateral [42]. Clinical and histological definitions aside, this model was based on accepted anthropometric data of the laryngeal geometry, and assumed transverse isotropy of the vocal folds. The nodules were simulated as masses near the supraglottis, along the center of the vocal fold length. Mass, size and anterior-posterior position were varied to assess the effects of these parameters on fundamental frequency.

During a preliminary study as part of this research [43, 44], vocal fold polyps were modeled as spherical protrusions from the medial surface of a finite element vocal fold model. The polyps were parameterized based on volume and position along the anterior-posterior length of the vocal fold. The vocal fold geometry was based on calibrated CT data published by Luo et al. [4], and was treated as a transversely isotropic 
material. Similarly to Jiang and Diaz [5], the polyp was treated as an isotropic material, due to its lack of fibers, with a fivefold increase in ligament stiffness. This model demonstrated that polyp position varied the fundamental frequency linearly. Fundamental frequency decreased as the polyp moved from the anterior position to the center of the vocal fold. An increase in polyp volume was shown to non-linearly decrease fundamental frequency of the vocal fold. This thesis is, in part, concerned with refining this model and performing more thorough analysis. Further examination of the interactions between position and volume is documented, and the effects of polyp shape and stiffness is considered.

\subsection{Objectives of the thesis}

This thesis is concerned with documenting the development of a parameterized, threedimensional finite element model of a vocal fold and polyp, and with the use of this model, performing the following numerical experiments:

1. Frequency-domain analysis will be performed through solution of the equation of motion under free vibration conditions. A three-dimensional model of a healthy vocal fold will be solved for its natural frequencies and modes of vibration, and subsequently compared against the pathological model. Sensitivity of the polyp's parameters will be assessed by examining interactions between polyp shape, mass, stiffness, and position.

2. Vocal folds have a pitch-intensity dependence. This has been roughly quantified as 2-6 $\mathrm{Hz} / \mathrm{cm} \mathrm{H}_{2} \mathrm{O}$ from excised, non-pathological larynges [45. Intensity is primarily controlled by subglottal pressure, and pitch is controlled in part by phonation frequency. To investigate the effect of pathology on this dependency, subglottal pressure will be varied, and phonation frequency will be approximated via 
transient solution of the equation of motion with a two-dimensional pathological vocal fold model. The change in this dependency due to the influence of pathologies is quantified.

\subsection{Structure of the Thesis}

The present chapter outlines the fundamentals of phonation by considering the physics governing aerodynamics in the larynx and vibration of the vocal folds. A chronological history of modeling vocal fold vibration and pathological tissues is presented.

Chapter 2 discusses the modeling approach taken by this study for the structural dynamics of the vocal folds and polyp. A finite element formulation of the equation of motion is presented, and its Fortran implementation is discussed. Results of a verification and validation study of the code is presented.

Chapter 3 presents the aerodynamic modeling approach taken by this study. Its coupling with the structural dynamics solver is discussed.

Chapter 4 outlines the results of two numerical studies. The first study examines the effect of polyp shape, mass, position, and stiffness on the frequencies and modes of vibration of the vocal folds. The second study examines the effect of a polyp on the pitch-intensity dependence of a vocal fold.

Chapter 5 gives a summary of the results of this study and recommendations for future work. 


\section{Chapter 2}

\section{Structural Dynamics Analysis}

\subsection{Introduction}

Modeling of the vocal fold and larynx are presented in this chapter. The choice of vocal fold geometry is presented and justified, and its associated material model is provided. A model of a unilateral, sessile polyp is then developed, and added to the vocal fold model. The governing equations of the structural dynamics are presented for both the frequency domain and time domain analysis. These equations are subsequently worked into a finite element framework, and solution methods are discussed. The implementation of the FEM formulation into software is outlined. Verification and validation of the results are presented with a comparison of existing literature models.

\subsection{Vocal Fold Model}

\subsubsection{Geometry}

Several models of vocal fold geometry exist, and are largely based on the body-cover theory of phonation. The use of body-cover theory is advantageous in the context of this work, as it presents a physiologically correct model which is still simple enough to 
parameterize and consequently study the effect of pathological or abnormal phonation [26]. Following a literature review, the geometric model presented in [4] was selected for the study of pathological phonation for the following reasons:

1. Realism: The model geometry is based on CT scan images |4|. Experimental details are not specified, apart from the use of polynomial functions to approximate surface geometry. Other models are typically idealized geometries, and as a consequence, feature linear, or smoothed surface geometries not typically observed in vocal folds.

2. Three layers: Three distinct materials are incorporated into the model: the body, cover, and ligament. This represents the most up to date interpretation of bodycover theory.

3. Polynomial description: The vocal fold's surface geometry is described using simple polynomial expressions for each layer.

An illustration of the vocal fold is given in Fig. 2.1. Mathematically, the surrounding surfaces of each layer is defined by the polynomial curves:

$$
\begin{aligned}
& y_{C}(x)= \begin{cases}2.304 x^{3}+0.552 x^{2} \quad:-0.8435 \leq x \leq 0 \\
-34.2 x^{2} & : 0<x \leq 0.107\end{cases} \\
& y_{L}(x)=\left\{\begin{array}{rr}
3.047 x^{3}+0.883 x^{2}+0.085 x-0.097 & :-0.7602 \leq x \leq-0.1 \\
-183.489 x^{3}-46.698 x^{2}-3.835 x-0.2 & :-0.1<x \leq 0.0859
\end{array}\right. \\
& y_{B}(x)= \begin{cases}4.388 x^{3}+1.509 x^{2}+0.170 x-0.194 & :-0.6796 \leq x \leq-0.1 \\
29.351 x^{3}-6.845 x^{2}-2.250 x-0.327 & :-0.1<x \leq 0.0376\end{cases}
\end{aligned}
$$




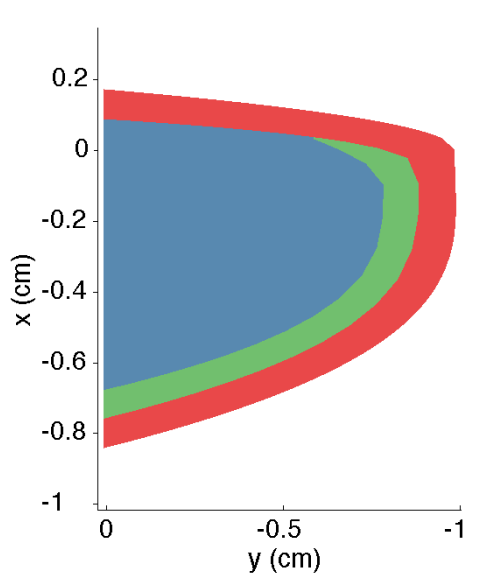

(a) Two-dimensional vocal fold geometry recreated using polynomial expressions

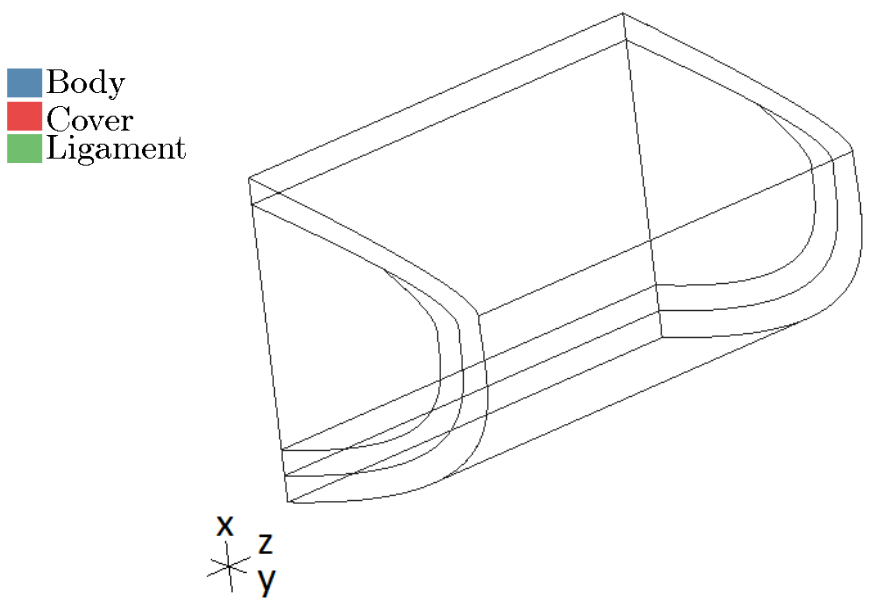

(b) Isometric wireframe vocal fold view

Figure 2.1: Vocal fold geometry based on CT data |4|

where the subscripts $C, L$, and $B$ denote the cover, ligament, and body layers, respectively, and $x$ and $y$ are the coordinates in $\mathrm{cm}|4|$. The $x-y$ plane in Fig.2.1(a) represents the coronal plane of the vocal fold. This geometry is extruded $1.4 \mathrm{~cm}$ in the $z$ direction, corresponding with the anterior-posterior direction, as shown in Fig.2.1(b).

Typically, the anterior-posterior geometry is considered to be curved [2,3,46], rather than linearly extruded, as adopted in this study. This choice was made for two reasons. The first is to simplify the analysis to medial-lateral and inferior-superior motion, i.e. the $x-y$, or coronal plane. Although modeling the geometry boundaries as curved would be more realistic, the characteristic motion considered in this study are motions related to abduction and adduction, and mucosal wave propagation, which are both observable from a coronal perspective. The second reason is for ease of polyp placement in the anterior-posterior position, which is documented in detail in Section 2.3. Adopting a symmetric model of a vocal fold will simplify the sensitivity analysis which will be performed with regards to polyp position. 


\subsubsection{Material behaviour}

The vocal folds are assumed to be subject to small deformations. The assumption of small deformation theory allows for a linear analysis to be performed, where stress is assumed to be linearly related to strain [47, p. 485]. Although vocal fold tissues have been experimentally shown to behave with nonlinear stress-strain relations, the development of a nonlinear constitutive model is difficult, due to extreme variation in experimentally measured material behaviour $[11]$, and the requirement of parameters which are impossible to estimate or measure [48].

In addition, vocal fold tissue behaviour is assumed to be transversely isotropic. The transverse plane is assumed to be in $x-y$. In the longitudinal direction, tissue fibres are assumed to lie along the $z$ axis, with separate material properties. This assumption is based on experimental data which shows a significant increase in stiffness in the anterior-posterior, or $z$-direction [2]. Properties of the transversely isotropic vocal fold model are taken from Luo et al. [4], which makes the same assumptions as the present work. The material properties of each constituent layer is summarized in Table 2.1. Transverse Youngs' moduli are calculated as 49

$$
\begin{aligned}
& E_{x}=2\left(1+v_{x y}\right) G_{x y} \\
& E_{y}=2\left(1+v_{y x}\right) G_{y x}
\end{aligned}
$$

and the longitudinal Poisson's ratios are calculated as

$$
\begin{aligned}
& v_{x z}=E_{x} \frac{v_{z x}}{E_{z}} \\
& v_{x z}=E_{y} \frac{v_{z y}}{E_{z}} .
\end{aligned}
$$

Viscoelastic values reported in Table 2.1 are taken from experimental measurements 
Table 2.1: Physical parameters for the three-layer transversely isotropic vocal fold |3|

\begin{tabular}{|l|l|l|l|}
\hline Parameter & Body & Cover & Ligament \\
\hline Transverse shear modulus, $G_{x y}, G_{y x}(\mathrm{kPa})$ & 1.05 & 0.53 & 0.87 \\
Longitudinal shear modulus, $G_{z}(\mathrm{kPa})$ & 12 & 10 & 40 \\
Longitudinal Young's modulus, $E_{z}(\mathrm{kPa})$ & 31.2 & 26 & 104 \\
Transverse Poisson ratio, $v_{x y}, v_{y x}$ & 0.3 & 0.3 & 0.3 \\
Longitudinal Poisson ratio, $v_{z x}, v_{z y}$ & 0.3 & 0.3 & 0.3 \\
Density, $\rho\left(\mathrm{g} / \mathrm{cm}^{3}\right)$ & 1.0 & 1.0 & 1.0 \\
\hline
\end{tabular}

from excised larynges [50-54]. Measurements for density were taken from excised canine larynges, which were found to be nearly constant for all three materials [51]. Of note, the densities reported are close to the density value of water $\left(1.0 \mathrm{~g} / \mathrm{cm}^{3}\right)$, which is expected for biological tissues, which are composed, in large part, by water.

There is some discussion in the relevant literature regarding the selection of the transverse Poisson's ratios for vocal fold modeling. The vocal fold tissue is thought to be incompressible due to a large portion of its composition being water [55]. Studies of the vocal fold eigenvectors and eigenmodes have also made use of compressible materials [2,4] by assuming Poisson ratios on the order of 0.3 , as is selected for this study. The justification for this selection is to simplify the analysis by focusing on the mediallateral and inferior-superior motion of the vocal folds. An incompressible selection of Poisson ratio would require additional analysis of the anterior-posterior motion, which is not considered in this study due to the selection of a symmetric vocal fold geometry. Accordingly, accepted values for compressible vocal fold tissues is adopted. 


\subsection{Pathological Modeling}

An idealized model of a polyp is difficult to develop due to the various shapes and sizes of polyps which develop on the vocal folds [42], as well as a lack of data regarding a polyp's material properties. Consequently, studies of computational polyp models have not examined major effects of its shape, stiffness, or position along the vocal fold anteriorposterior length on phonation. To examine such effects, this work models a sessile polyp as an ellipsoid hemisphere which protrudes from the midpoint of the coronal surface of the vocal fold into the glottis. The model is parameterized based on the variation of the polyp's shape, mass, stiffness, and position along the anterior-posterior direction.

1. Shape: The polyp may be altered by varying the size of the minor axes of the ellipsoid, $A, B$, and $C$, illustrated in Fig. 2.2. The equation for the closed surface of an ellipsoid is given by

$$
\left(\frac{z}{A}\right)^{2}+\left(\frac{x}{B}\right)^{2}+\left(\frac{y}{C}\right)^{2}=1
$$

where $A$ varies the length in the anterior-posterior direction, $B$ varies the length in the inferior-superior direction, and $C$ varies the length in the medial-lateral direction. Defining the polyp in this way allows an examination of the effects of the polyp's various aspect ratios on phonation properties. The limits of $A, B$, and $C$

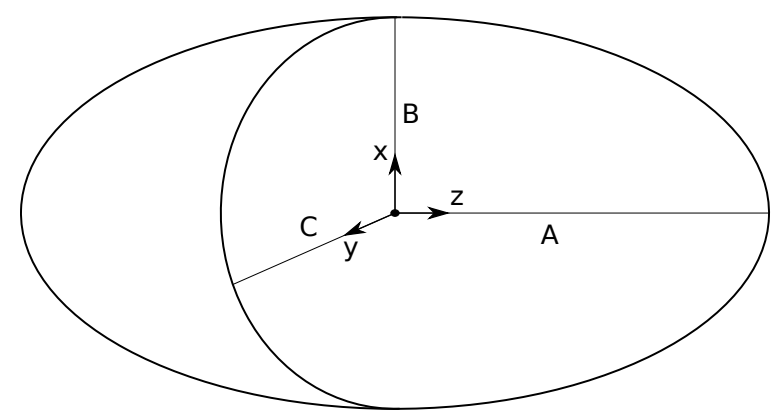

Figure 2.2: Ellipsoid hemisphere model of a polyp, and the minor axes parameters. 
are based on a clinical study [42], which gives results of a clinical survey of patients who presented with vocal fold polyps, suggesting a range between $0.5 \mathrm{~mm}$ and $3.5 \mathrm{~mm}$ in each direction.

2. Mass: The mass of the polyp is varied based on the selection of uniform polyp density, $\rho_{p}=1.30 \mathrm{~g} / \mathrm{cm}^{3}$, and its volume. Volume is controlled by adjusting the three minor axes of the ellipsoid. Based on the volume of half of an ellipsoid, $V=$ $\frac{2}{3} \pi A B C$, the mass of the polyp is given as $m_{p}=\frac{2}{3} \pi A B C \rho_{p}$.

3. Stiffness: A vocal fold polyp is histologically defined as being composed of a gelatinous material [28]; it distinguishes itself from the vocal fold material by its lack of tissue fibers. Consequently, the polyp will be modeled as an isotropic material, requiring physiological parameters for its Young's modulus, $E_{p}$, and Poisson's ratio, $v_{p}$. It is understood that the vocal fold polyp exhibits an increase in stiffness compared to the surrounding vocal fold tissue. Jiang et al. [5] model the Young's modulus of the polyp as a fivefold increase in longitudinal stiffness of the ligament, $E_{L}$, the highest Young's modulus value used in the model, based on the observation that the polyp will present with an increase in stiffness. The assumption of this increase being quantified as a fivefold increase is not justified. Accordingly, stiffness is varied in the polyp based on the parameter $\alpha_{p}$, which is used to define the polyp's Young's modulus as $E_{p}=\alpha_{p} E_{L}$. This parameter is varied within the range of $2 \leq \alpha_{P} \leq 5$.

4. Position: The polyp's center, $c$, may be moved in the anterior-posterior direction. The center of the vocal fold is set as reference $c=0$. The range of $c$ is varied between $0 \mathrm{~mm} \leq c \leq 7 \mathrm{~mm}$, as illustrated in Fig. 2.3 . 


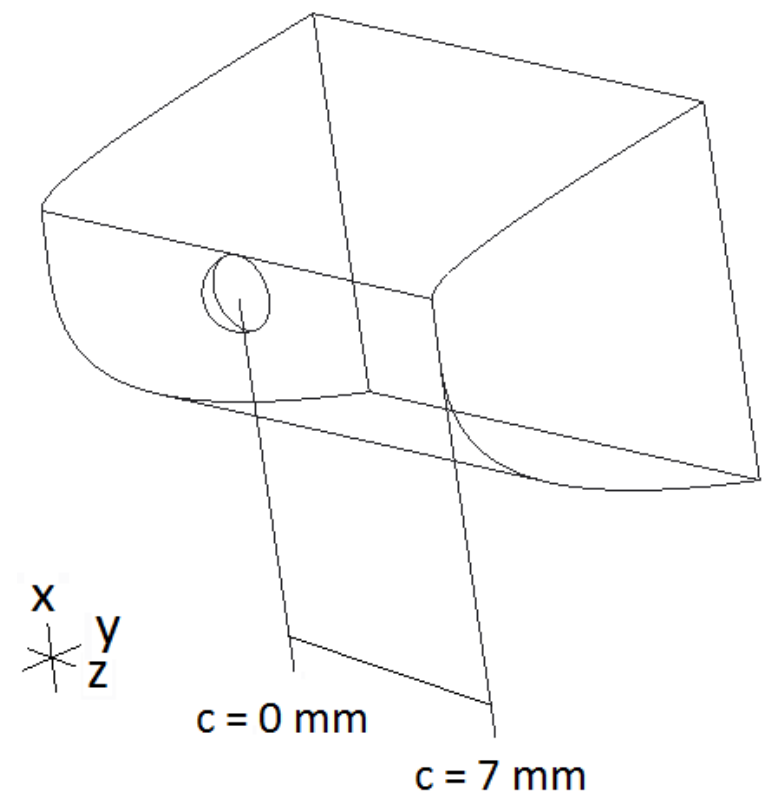

Figure 2.3: Range of polyp position parameter, $c$.

A further modeling consideration of a polyp is the point of insertion of the polyp on the vocal fold model. Polyps form on the epithelial layer of the vocal fold [28]. Therefore, the polyp is placed on top of the cover layer, and any of the elliptical polyp's geometry which intersects the vocal fold tissue is removed.

Finally, the remaining material parameter to define is the polyp's Poisson's ratio. There is a lack of experimental data which may be used to define this parameter. It is assumed that the polyp will behave similarly to the underlying vocal fold tissue. Table 2.1 illustrates that in all directions, the Poisson's ratio is uniformly 0.3 . This value will be used to define the polyp's Poisson ratio, $v_{p}=0.3$. Table 2.2 summarizes the isotropic material properties of the polyp model, and Table 2.3 summarizes the range of values used for the shape, stiffness, and positioning. 
Table 2.2: Unilateral sessile polyp model material properties

\begin{tabular}{|l|l|}
\hline Parameter & Value \\
\hline Young's Modulus, $E_{p}(\mathrm{kPa})$ & $\alpha_{P} E_{L}$ \\
Poisson's ratio, $v_{p}$ & 0.3 \\
Density, $\rho_{p}\left(\mathrm{~g} / \mathrm{cm}^{3}\right)$ & 1.03 \\
\hline
\end{tabular}

Table 2.3: Unilateral sessile polyp model parameters

\begin{tabular}{|l|l|l|}
\hline Parameter & Lower limit & Upper limit \\
\hline$A(\mathrm{~mm})$ & 0.5 & 3.5 \\
$B(\mathrm{~mm})$ & 0.5 & 3.5 \\
$C(\mathrm{~mm})$ & 0.5 & 3.5 \\
$\alpha_{P}$ & 2 & 5 \\
$c(\mathrm{~mm})$ & 0 & 3.5 \\
\hline
\end{tabular}

\subsection{Structural Analysis}

The vibration of a vocal fold system does not have an analytical solution due to its complex geometry and material composition. Consequently, an approximate solution is sought by formulating its equation of motion using a numerical method for a model. Various techniques exist to formulate a discretised system's equation of motion, however, the finite element method offers several distinct advantages:

1. Lagrangian field specification: The structure's equations will be formulated from a Lagrangian frame of reference, where the structure deforms relative to a stationary coordinate frame. For time-dependent solutions of the equation of motion, where the mesh will deform as the solution is marched in time, the finite element method easily accommodates mesh motion.

2. Complex domain geometry: The finite element method accommodates the use of a mesh over an arbitrarily shaped domain. The base finite element code remains 
virtually unchanged for different meshes, whereas other methods may require grid transformations or more complicated data structures.

3. Support of multiple materials within a single domain: The distinction between multiple materials within a domain is simple to accommodate with the finite element method, which considers each element as having its own material properties. In this context, each element will be treated as either a body, cover, or ligament layer.

\subsubsection{Governing equations}

From Newton's second law under dynamic equilibrium, a system's time-dependent history of motion $\mathbf{q}(x, y, z, t)$ in Newton's notation is given as

$$
[M] \ddot{\mathbf{q}}+[C] \dot{\mathbf{q}}+[K] \mathbf{q}=[\mathbf{F}]
$$

where $[M]$ is the inertial mass matrix, $[C]$ is the damping matrix, and $[K]$ is the stiffness matrix of the system [47, p. 499]. This equation represents a force balance on a system of discrete connected masses with applied forces [56, p. 2], as illustrated in Fig. 2.4. The finite element method is used to develop relations for $[M],[C]$, and $[K]$ in our discrete model, and subsequently solve this system for $\mathbf{q}, \dot{\mathbf{q}}$, and $\ddot{\mathbf{q}}$ in the time domain.

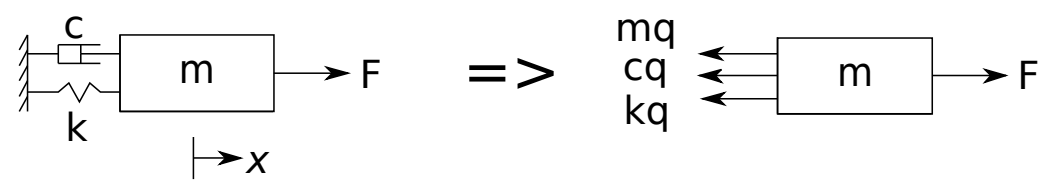

Figure 2.4: One-dimensional mass-spring-damper system, and its dynamic equilibrium force diagram analogue. 
In addition to the time-dependent history of the system, this work is also concerned with characterizing vibration using natural frequencies, $\omega$ and modes of vibration, $\phi$. Such parameters are properties of the system under free-vibration conditions, in which damping is neglected, and no forces are applied. Under these conditions, Eq.2.5 reduces to

$$
[M] \ddot{\mathbf{q}}+[K] \mathbf{q}=0 .
$$

We assume a solution to Eq.2.6 in the form

$$
\mathbf{q}=\phi \sin \omega\left(t-t_{0}\right)
$$

where $t$ is the time variable, and $t_{0}$ is a time constant. Substituting Eq. 2.7 into Eq. 2.6. we obtain the eigensystem

$$
[K] \phi=\omega^{2}[M] \phi
$$

which is solved for $\omega$ and $\phi$ [56, p. 57].

\subsubsection{FEM formulation}

Eq. 2.5 and Eq.2.8 represent a variational problem, wherein multiple solutions may satisfy the equations for a given set of boundary and initial conditions. This concept is illustrated in Fig. 2.5, where an infinite number of paths can be taken by a particle between two positions. Hamilton's principle is used to address this issue by adding a new condition to the system, which states that the Lagrangian, or energy functional of the system, must be minimized [47, p. 110]. Hamilton's principle is stated as

$$
\int_{t_{2}}^{t_{2}}\left(\delta(T-U)+\delta W_{n c}\right) d t=0
$$


where $T$ represents the scalar kinetic energy functional, $U$ represents the potential energy, or strain energy functional, $W_{n c}$ represents the summation of non-conservative forces on the system, and $\delta$ is a virtual displacement term. This equation is a re-iteration of the equation of dynamic equilibrium in a scalar energy format, positing that the sum of kinetic and potential energy of a system must be balanced against applied forces 56 . p. 53].

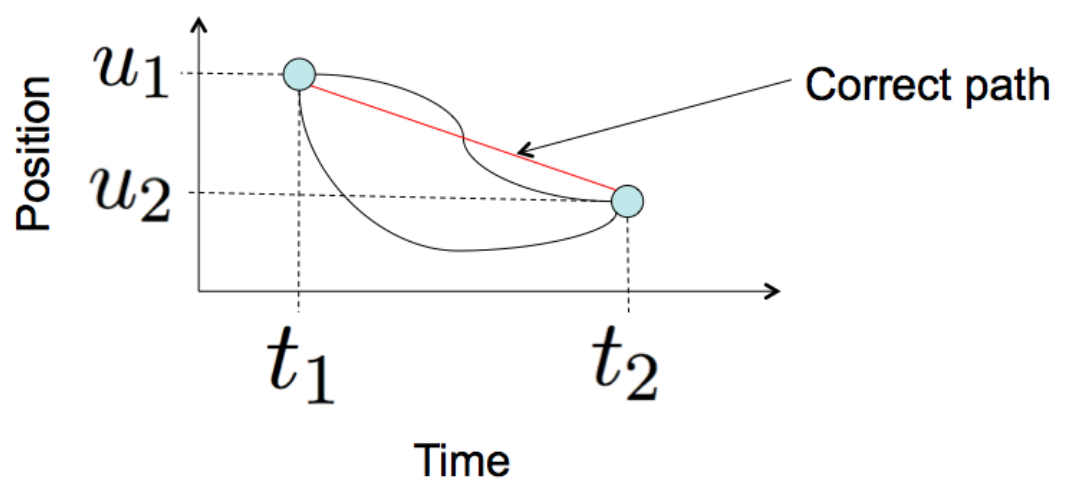

Figure 2.5: Hamilton's principle, or the principle of least action. Scalar energy functionals are used to distinguish the correct path of the particle between $u_{1}$ and $u_{2}$.

Solutions of Eq.2.9 are found using the Rayleigh-Ritz method, which develop expressions of the individual energy functionals $T$, and $U$. These scalar energy functionals are expressed as

$$
T=\frac{1}{2} \int_{V} \rho \dot{\mathbf{q}} d V
$$

where $V$ is the volume of the moving mass, $\rho$ is its density, and

$$
U=\frac{1}{2} \int_{V} \sigma \epsilon d V=\frac{1}{2} \int_{V} \epsilon^{T}[D] \epsilon d V
$$

where $\sigma$ is the stress vector, $\epsilon$ is the strain vector. $\epsilon$ may be re-expressed as

$$
\epsilon=\left\{\begin{array}{lllll}
\epsilon_{x x}, & \epsilon_{y y}, \quad \epsilon_{z z}, \quad \epsilon_{y z}, \quad \epsilon_{z x}, \quad \epsilon_{x y}
\end{array}\right\}^{T},
$$


where $\epsilon_{i j}$ represents the strain component in tensor notation. Stress is linearly related to strain as

$$
\sigma=[D] \epsilon
$$

where $[D]$ may contain up to 21 material constants to represent deformation behaviour of the system in all directions [56, pp. 37-39]. Specifically for the transversely isotropic material considered, five material constants are required to define $[D]$.

Eq. 2.10 and Eq. 2.11 are substituted into Eq.2.9. Through algebraic manipulation, it can be shown that

$$
\begin{gathered}
\frac{\partial}{\partial t} \frac{\partial T}{\partial \dot{\mathbf{q}}}=[M] \ddot{\mathbf{q}}, \\
\frac{\partial U}{\partial \mathbf{q}}=[K] \mathbf{q} .
\end{gathered}
$$

Eq. 2.14 and Eq.2.15are Lagrange's equations, which are scalar energy functions formulated in terms of $\mathbf{q}$ to represent the constitutive terms of the equation of motion [56, $\mathbf{p}$. 13]. The finite element method is now used to develop the equation of motion for the multi degree of freedom system by formulating Lagrange's equations for each discrete element in the model, and assembling the global system of equations.

The finite element method splits the domain into a collection of elements, which are defined by nodes. An example of this discretization is illustrated in Fig. 2.6. Element sizes are distributed uniformly where possible, and decreased near the border of the three materials. Values of $\mathbf{q}$ are stored at the nodes. To define $\mathbf{q}$ within the domain, element-specific shape functions, denoted $[N]$, are used to interpolate for $\mathbf{q}$ within the element based on its nodal values, denoted $\mathbf{q}^{e}$. The position vector $\mathbf{q}$ can thus be reexpressed

$$
\mathbf{q}=\sum_{k=1}^{n}[N] \mathbf{q}_{k}^{e}
$$



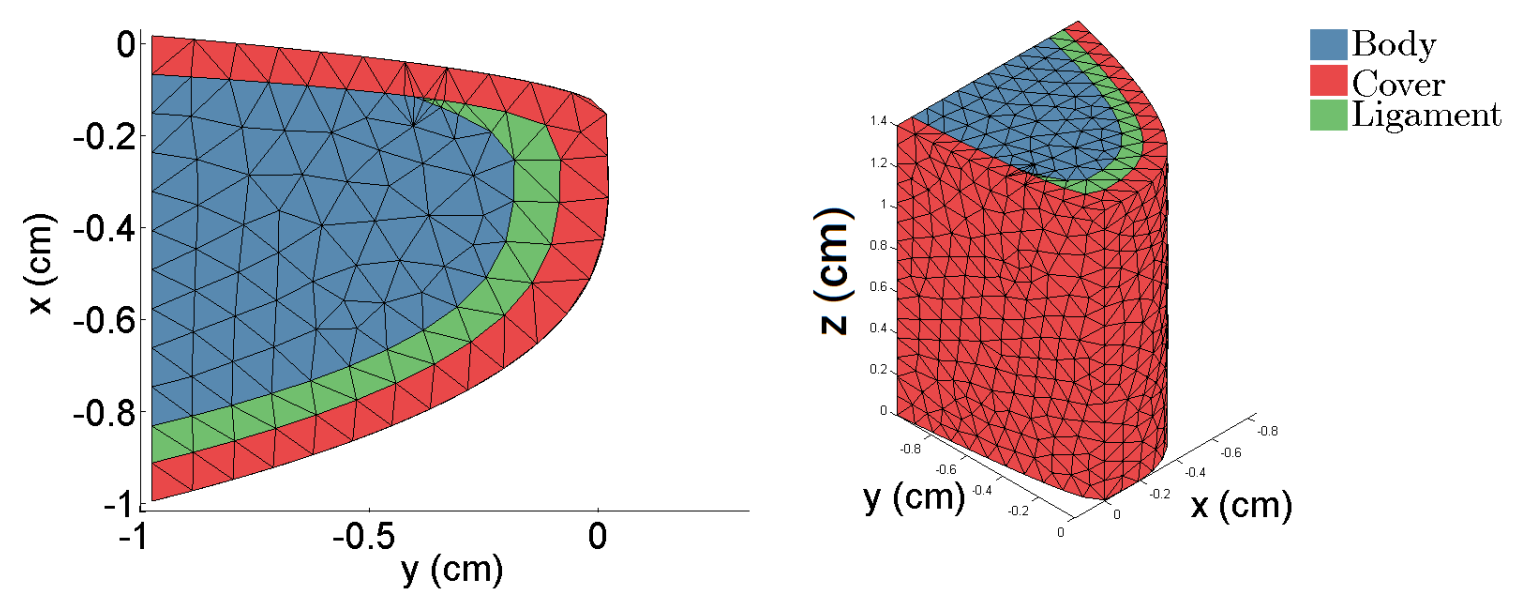

Figure 2.6: Three-dimensional vocal fold is discretized using tetrahedral elements. Values of $\mathbf{q}$ are stored at node points, and other values within the field are interpolated using element specific shape functions.

where $n$ is the number of nodes required to define the element [70].

Eq.2.16 is substituted into Lagrange's equations, Eq.2.14 and Eq.2.15 and evaluated. The element matrices are thus defined as

$$
m_{e}=\int_{V} \rho[N]^{T}[N] d V
$$

and

$$
k_{e}=\int_{V}[B]^{T}[D][B] d V
$$

where $m_{e}$ is the element mass matrix, $k_{e}$ is the element stiffness matrix 47, pp. 124-125], and $[B]$ is the element strain matrix, given by $|70|$

$$
[B]=[\nabla[N], \nabla \times[N]]^{T} .
$$

This work uses four-node tetrahedral elements and three node plane strain triangular elements to discretize the vocal fold system, as shown in Fig.2.7(a) and Fig.2.7(b) respectively. A preliminary study as part of this work showed that higher order elements offered 


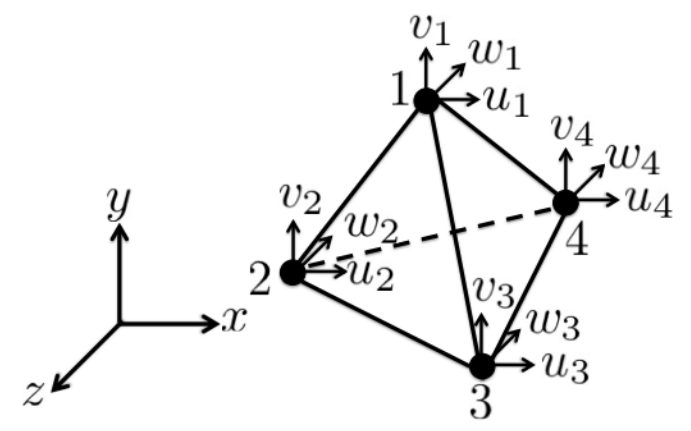

(a) Linear (4-node) tetrahedral element, with 12 degrees of freedom.

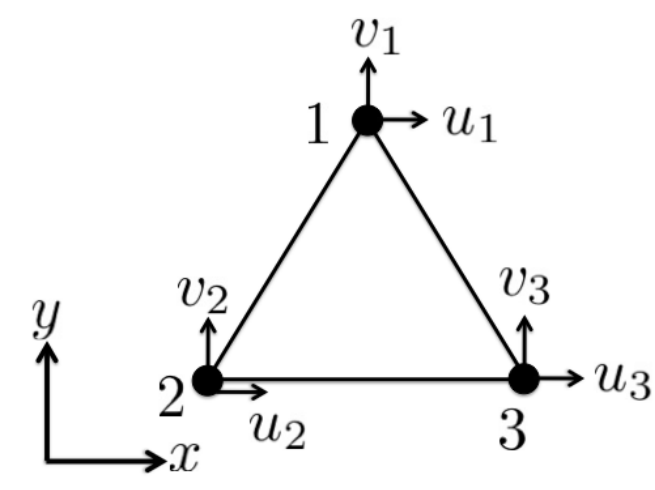

(b) Linear (3-node) triangular element, with 6 degrees of freedom

Figure 2.7: Elements incorporated in the vocal fold models.

better accuracy, but increased the computation time. Based on the minimal differences between respective solutions, linear elements were selected for their efficiency [44]. The sections which follow outline the derivation of the finite element equations for the tetrahedral element. The derivation for the triangular element is very similar, and accordingly, is given in Appendix A.

\subsubsection{Deriving the four-node tetrahedral shape functions}

The purpose of the shape functions is to interpolate for field variables within the domain defined by the element. The shape functions define, for the set of all nodes in the element, a function which is continuous within the element domain, is equal to unity at its corresponding node, and zero at all other nodes [56, p. 63]. The tetrahedral element uses its volume coordinates to define $[N]$. To demonstrate, we define a point within the tetrahedral which is not coincident with any tetrahedral face. In Fig. 2.8, this point is shown distinct from the nodes as a shaded circle. The tetrahedral 1234 may now be 
separated into four sub-tetrahedrals with their own distinct volume. We adopt the convention $V_{i}$ to define the volume of the sub-tetrahedral which does not have a vertex at node $i$.
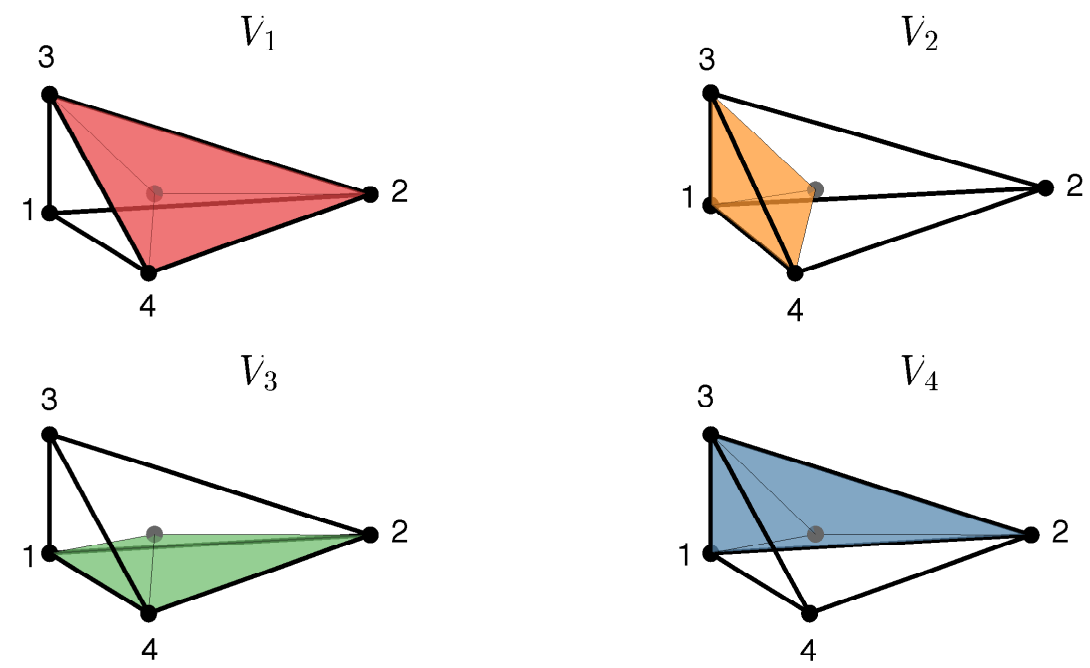

Figure 2.8: Sub-tetrahedrals defined by an arbitrary point within the element.

We may now express the volume of tetrahedral $1234, V$, as

$$
\begin{aligned}
\sum_{i=1}^{4} V_{i}=V \\
\Longrightarrow \sum_{i=1}^{4} \frac{V_{i}}{V}=1 \\
\Longrightarrow \sum_{i=1}^{4} L_{i}=1
\end{aligned}
$$


where $L_{i}$ are the volume coordinates of the tetrahedral [56]. Cartesian and volume coordinates are related as follows [56]:

$$
\begin{aligned}
& x=x_{1} L_{1}+x_{2} L_{2}+x_{3} L_{3}+x_{4} L_{4} \\
& y=y_{1} L_{1}+y_{2} L_{2}+y_{3} L_{3}+y_{4} L_{4} \\
& z=z_{1} L_{1}+z_{2} L_{2}+z_{3} L_{3}+z_{4} L_{4} .
\end{aligned}
$$

Combining Eq. 2.20 and Eq.2.21, we develop the following system of equations [56]

$$
\left[\begin{array}{l}
1 \\
x \\
y \\
z
\end{array}\right]=\left[\begin{array}{cccc}
1 & 1 & 1 & 1 \\
x_{1} & x_{2} & x_{3} & x_{4} \\
y_{1} & y_{2} & y_{3} & y_{4} \\
z_{1} & z_{2} & z_{3} & z_{4}
\end{array}\right]\left[\begin{array}{c}
L_{1} \\
L_{2} \\
L_{3} \\
L_{4}
\end{array}\right] .
$$

To obtain an expression for the volume coordinates, $L_{i}$, as a function of the nodal coordinates, $\left(x_{i}, y_{i}, z_{i}\right)$, we take the inverse of Eq.2.22. We adopt the convention

$$
\begin{aligned}
& x_{i j}=x_{i}-x_{j} \\
& y_{i j}=y_{i}-y_{j} \\
& z_{i j}=z_{i}-z_{j} .
\end{aligned}
$$


Taking the inverse of Eq. 2.22 ,

$$
\left[\begin{array}{l}
L_{1} \\
L_{2} \\
L_{3} \\
L_{4}
\end{array}\right]=\frac{1}{6 V}\left[\begin{array}{cccc}
6 V_{1} & y_{42} z_{32}-y_{32} z_{42} & x_{32} z_{42}-x_{42} z_{32} & x_{42} y_{32}-x_{32} y_{42} \\
6 V_{2} & y_{31} z_{43}-y_{34} z_{13} & x_{43} z_{31}-x_{13} z_{34} & x_{31} y_{43}-x_{34} y_{13} \\
6 V_{3} & y_{24} z_{14}-y_{14} z_{24} & x_{14} z_{24}-x_{24} z_{14} & x_{24} y_{14}-x_{14} y_{24} \\
6 V_{4} & y_{13} z_{21}-y_{12} z_{31} & x_{21} z_{13}-x_{31} z_{12} & x_{13} y_{21}-x_{12} y_{31}
\end{array}\right]\left[\begin{array}{l}
1 \\
x \\
z
\end{array}\right] .
$$

we define an expression for the volume coordinates as a function of the nodal coordinates, and the coordinates of a point within the domain [57].

Since the volume coordinates satisfy the conditions required for shape functions,

$$
N_{i}=L_{i}
$$

Subsequently, we define the displacement functions using the shape functions as interpolation factors,

$$
\begin{gathered}
u=\sum_{i=1}^{4} N_{i} u_{i} \\
v=\sum_{i=1}^{4} N_{i} v_{i} \\
w=\sum_{i=1}^{4} N_{i} w_{i}
\end{gathered}
$$

where $u, v$, and $w$ are the displacements in the $x, y$, and $z$ directions, respectively, and 
$u_{i}, v_{i}$, and $w_{i}$ are the displacements at node $i[70 \mid$. We re-write this expression

$$
\{\mathbf{q}\}=[N]\left[\begin{array}{c}
u \\
v \\
w
\end{array}\right]=[N]\left\{\mathbf{u}_{e}\right\}
$$

where

$$
\left\{\mathbf{u}_{e}\right\}=\left\{\begin{array}{lllllll}
u_{1} & v_{1} & w_{1} & \cdots & u_{4} & v_{4} & w_{4}
\end{array}\right\}^{T}
$$

and

$$
[N]=\left[\begin{array}{ccccccc}
L_{1} & 0 & 0 & \cdots & L_{4} & 0 & 0 \\
0 & L_{1} & 0 & \cdots & 0 & L_{4} & 0 \\
0 & 0 & L_{1} & \cdots & 0 & 0 & L_{4}
\end{array}\right]
$$

which defines the shape function matrix for the linear tetrahedral element [56, p. 215].

\subsubsection{Deriving the element mass matrix}

Now that $[N]$ has been defined in Eq. 2.29 , the element mass matrix defined in Eq.2.17 may be developed. Typically, this matrix is evaluated using numerical integration, however, in this case, the integral may be solved for the general case [57]. Matrix entry $i, j$ in the integrand of Eq.2.17] is of the form

$$
\left[m_{e}\right]_{i, j}=\rho \int_{V} L_{k} L_{r} d V \quad \text { for } k=1,2,3,4 \text {, and } r=1,2,3,4 \text {. }
$$


This integral may be evaluated using the formula [56]

$$
\left[m_{e}\right]_{i, j}=\rho \int_{V} L_{1}^{m} L_{2}^{n} L_{3}^{p} L_{4}^{q} d V=\rho \frac{m ! n ! p ! q !}{(m+n+p+q+3) !} 6 V
$$

which can be simplified to [56]

$$
\left[m_{e}\right]_{i, j}=\rho L_{k} L_{r}=\left\{\begin{array}{cc}
\frac{\rho V}{10} & : k=r \\
\frac{\rho V}{20} & : k \neq r
\end{array} .\right.
$$

The element mass matrix may now be evaluated for each entry using Eq.2.32,

\subsection{Solution Methods}

To develop the global system of FEM equations for Eq. 2.5 and Eq. 2.6, $m_{e}$ and $k_{e}$ are computed for each element, and mapped into the global system based on the degrees of freedom specified in q. Each element generates a total of $3 n$ equations, where $n$ is the number of nodes which define the element. These $3 n$ equations correspond with one equation per degree of freedom of the element ( 3 translational degrees of freedom per node). The assembly of $[M]$ and $[K]$ constitutes the development of large, sparse, banded matrices. Thus, the solution of Eq.2.5 and Eq. 2.6 requires consideration of the numerical methods selected to balance accuracy and efficiency.

\subsubsection{Direct integration of the equation of motion}

Eq. 2.5 requires numerical integration for a time-dependent solution of q. The method selected is the Newmark-Beta scheme. This method assumes that over an interval $\Delta t$, acceleration varies linearly. Two relations for $\dot{\mathbf{q}}$ and $\ddot{\mathbf{q}}$ are adopted, using the subscript 
convention $t$ to refer to the current time, and $t+1$ to refer to time $t+\Delta t$ :

$$
\begin{gathered}
\dot{\mathbf{q}}_{t+1}=\dot{\mathbf{q}}_{t}+(1-\gamma) \ddot{\mathbf{q}}_{t} \Delta t+\gamma \ddot{\mathbf{q}}_{t+1} \Delta t \\
\mathbf{q}_{t+1}=\mathbf{q}_{t}+\dot{\mathbf{q}}_{t} \Delta t+\left(\frac{1}{2}-\beta\right) \ddot{\mathbf{q}}_{t} \Delta t^{2}+\beta \ddot{\mathbf{q}}_{t+1} \Delta t^{2}
\end{gathered}
$$

where $\gamma$ and $\beta$ are parameters which define how much $\ddot{\mathbf{q}}_{t+1}$ enters into the relations for $\dot{\mathbf{q}}_{t+1}$ and $\mathbf{q}_{t+1}\left[58\right.$. Newmark showed that $\gamma=\frac{1}{2}$ to avoid numerical damping. A choice of $\beta=\frac{1}{4}$ results in a direct integration scheme which is unconditionally stable for any choice of $\Delta t$. Inspection of Eq. 2.34 for this selection of $\gamma$ and $\beta$ reveals that such an assumption is equivalent to the trapezoidal method [47, p.780].

An additional equation is required to establish a system of three equations for the three unknowns, $\ddot{\mathbf{q}}_{t+1}, \dot{\mathbf{q}}_{t+1}$, and $\mathbf{q}_{t+1}$. Accordingly, at time $t+\Delta t$, Eq. 2.5 becomes

$$
[M] \ddot{\mathbf{q}}_{t+1}+[C] \dot{\mathbf{q}}_{t+1}+[K] \mathbf{q}_{t+1}=[F]_{t+1} .
$$

The Newmark equations are now algebraically manipulated for each of the unknowns. Eq. 2.34 may be re-arranged as

$$
\ddot{\mathbf{q}}_{t+1}=\frac{1}{\beta \Delta t^{2}}\left(\mathbf{q}_{t+1}-\mathbf{q}_{t}\right)-\frac{1}{\beta \Delta t} \dot{\mathbf{q}}_{t}-\left(1-\frac{1}{2 \beta}\right) \ddot{\mathbf{q}}_{t} .
$$

Eq. 2.33 and Eq.2.36 are substituted into Eq.2.35 to obtain an expression for $\mathbf{q}_{t+1}$ :

$$
[\hat{K}] \mathbf{q}_{t+1}=[\hat{F}]
$$

where

$$
[\hat{K}]=[K]+\frac{1}{\beta \Delta t^{2}}[M]+\frac{\gamma}{\beta \Delta t}[C]
$$


and

$$
\begin{gathered}
{[\hat{F}]=[F]_{t+1}+\left[\frac{1}{\beta \Delta t^{2}} \mathbf{q}_{t}+\frac{1}{\beta \Delta t} \dot{\mathbf{q}}_{t}+\left(\frac{1}{2 \beta}-1\right) \ddot{\mathbf{q}}_{t}\right][M]+\ldots} \\
\ldots+\left[\frac{\gamma}{\beta \Delta t} \mathbf{q}_{t}+\left(\frac{\gamma}{\beta}-1\right) \dot{\mathbf{q}}_{t}+\frac{\Delta t}{2}\left(\frac{\gamma}{\beta}-2\right) \ddot{\mathbf{q}}_{t}\right][C]
\end{gathered}
$$

Assuming prior knowledge of $\ddot{\mathbf{q}}_{t}, \dot{\mathbf{q}}_{t}$, and $\mathbf{q}_{t}$, direct integration is now performed for time $t+\Delta t$ as follows:

1. Solve Eq.2.37 for $\mathbf{q}_{t+1}$.

2. Solve Eq.2.36 for $\ddot{\mathbf{q}}_{t+1}$.

3. Solve Eq. 2.33 for $\dot{\mathbf{q}}_{t+1}$.

The selection of $\Delta t$ is based on the highest frequency to be included in the solution. First, Eq. 2.8 is solved for $\omega$. The period of the largest natural frequency to be computed, $\omega_{i}$, is determined as

$$
T_{i}=\frac{2 \pi}{\omega_{i}}
$$

Finally, $\Delta t$ is selected based on applying a sampling factor of 20 [4], that is

$$
\Delta t=\frac{1}{20} T_{i}
$$

The timestep selection for the Newmark-Beta method is quite conservative due to it's time order accuracy of $\mathscr{O}(\Delta t)$. For first order accuracy in time, a conservative timestep is required in order for the solution to approach convergence. Less conservative methods may be selected by using higher order methods, such as second order central difference or fourth order Runge-Kutta. The justification for the selection of the first order Newmark-Beta method is its unconditional stability. By comparison, higher order methods require careful selection of the timestep in order to match a stability criterion, such 
as the Courant-Friedrichs-Lewy condition for central difference.

\subsubsection{Solution of the Eigenproblem}

The solution of the eigenproblem presented in Eq. 2.8 requires the solution of a large, symmetric, real, generalized eigenvalue problem. Numerous solution algorithms are documented in literature, however, in the interest of computational efficiency, there is significant merit in making use of pre-written subroutines which are optimized for the specific task. LAPACK (Linear Algebra PACKage) is the joint effort of numerous contributors in the computational mathematics community to develop a highly-optimized linear algebra software library [59]. This work makes use of the subroutine "DGGEV" contained within the library, which is designed for double precision computation of the eigenvalues, $\lambda$, and eigenvectors, $x$, of the $N$ by $N$ non-symmetric matrices $A$ and $B$ which constitute the system $A x=\lambda B x$.

DGGEV solves for $\lambda$ and $x$ of $A$ and $B$ by performing a generalized Schur decomposition on $A$ and $B$. Note that the discussion of Schur decomposition in this work is limited to the real case; complex solutions were not computed in this study. Schur decomposition is of the form

$$
\begin{aligned}
& A=Q S Z^{T} \\
& B=Q T Z^{T}
\end{aligned}
$$

where $Q$ and $Z$ are orthogonal matrices, and $S$ and $T$ are upper triangular, and lower triangular matrices, respectively. Note, that due to the orthogonality of $Q$ and $Z, Z^{T}=$ $Q^{-1}$, and

$$
\begin{aligned}
& A=Q S Q^{-1} \\
& B=Q T Q^{-1}
\end{aligned}
$$

Thus, $A$ is similar to $S$, and $B$ is similar to $T$. Therefore, the set of $\lambda$ for $A$ will be shared by $S$, and likewise for $B$ and $T$. The resulting diagonals of $S$ and $T$ are denoted $\alpha$ and $\beta$, 
respectively. The corresponding $N$ eigenvalues of the system are then computed as

$$
\lambda_{i}=\frac{\alpha_{i}}{\beta_{i}}
$$

Schur decomposition is performed with the QR iteration algorithm. For the matrix $A$, its $\mathrm{QR}$ decomposition is defined by $A=Q R$, where $Q$ is an orthogonal matrix. The similarity argument can be made that

$$
Q R=A=Q^{T} A Q=Q^{T}(Q R) Q=\left(Q^{T} Q\right)(R Q)=R Q
$$

which demonstrates similarity of $Q R$ and $R Q$. This similarity is exploited by recursively $\operatorname{defining} A^{(k)}, Q^{(k)}$, and $R^{(k)}$ :

$$
\begin{aligned}
Q^{(k)} R^{(k)} & =A^{(k-1)} \\
A^{(k)} & =R^{(k)} Q^{(k)}
\end{aligned}
$$

where $k$ is the number of iterations performed $[60]$. It can be shown that

$$
\lim _{k \rightarrow \infty} A^{(k)}=S
$$

and, similarly for $B$,

$$
\lim _{k \rightarrow \infty} B^{(k)}=T
$$

The computation of the QR decomposition of A and B is performed using a Householder transformation [59]. 


\subsubsection{Boundary and initial conditions}

Degrees of freedom in the vocal fold model may be either active, or fixed. A fixed node $i$ must maintain the Dirichlet boundary condition

$$
\mathbf{q}_{i}=0
$$

The simplest method of enforcing the fixed condition is to neglect mapping the element mass and stiffness values to the global system of equations. Consequently, only the active degrees of freedom are influential within the numerical solvers, and the fixed node condition is implicitly enforced.

On the vocal fold, the anterior and posterior planes (the $x-y$ planes) and the lateral face $(x-z$ surface) are held fixed, as illustrated in Fig. 2.9. These surfaces are attached to cartilage |4], which has the effect of damping vibration.

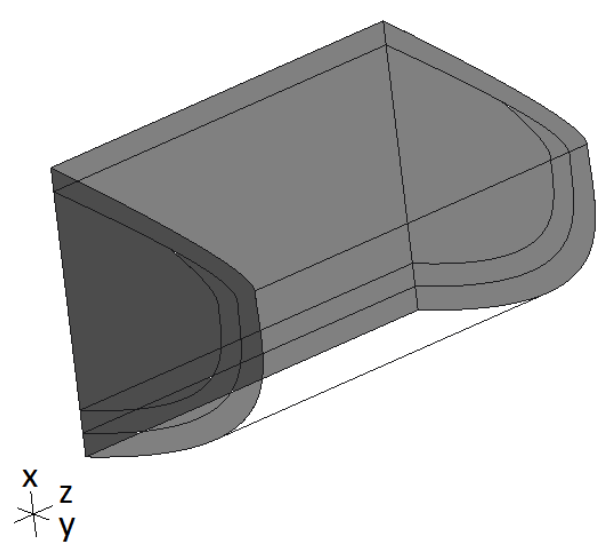

Figure 2.9: Fixed planes on the vocal fold. Nodes on the anterior and posterior planes, and the lateral surface are fixed.

The transient structural dynamics solution requires initial conditions for $\mathbf{q}$, $\dot{\mathbf{q}}$, and 苂. At time $t=0$, the assumption is made that the vocal fold is initially at rest, implying 
$\mathbf{q}_{0}=0$ and $\dot{\mathbf{q}}_{0}=0$. From Eq.2.5, the initial acceleration is estimated by computing

$$
\ddot{\mathbf{q}}_{0}=\frac{[F]_{0}}{[M]},
$$

which is computed prior to Newmark Beta integration [47].

For transient simulations with the two-dimensional model, contact between the vocal folds must be addressed. Simulation of contact with the finite element method presents a new dimension of complexity. The model must have a means of detecting collision and computing contact forces, both of which are governed by a separate mathematical framework for piecewise-smooth dynamics [61]. This aspect of simulation is outside of the scope of the present work, and accordingly, special treatment is required. Several finite element models make use of a rigid contact line which the vocal fold is unable to move past [3, 37, 62]. This allows for collision to be simulated kinematically. The position of the contact line is illustrated in Fig. 2.10, If a node $i$ is detected to move across this line, located at coordinate $y_{c}$,

$$
\begin{aligned}
& \mathbf{q}_{i}=y_{c}-y_{i} \\
& \ddot{\mathbf{q}}_{i}=0
\end{aligned}
$$

where $y_{i}$ is the coordinate of node $i$ at the previous time level. This has the effect of keeping the vocal fold "attached" at the contact line during the abduction phase.

\subsection{Implementation}

The simulations are implemented using in-house coding due to the use of nontraditional boundary and forcing conditions. When using a commercial software package, there are always limitations in implementing user-defined subroutines. Although a 


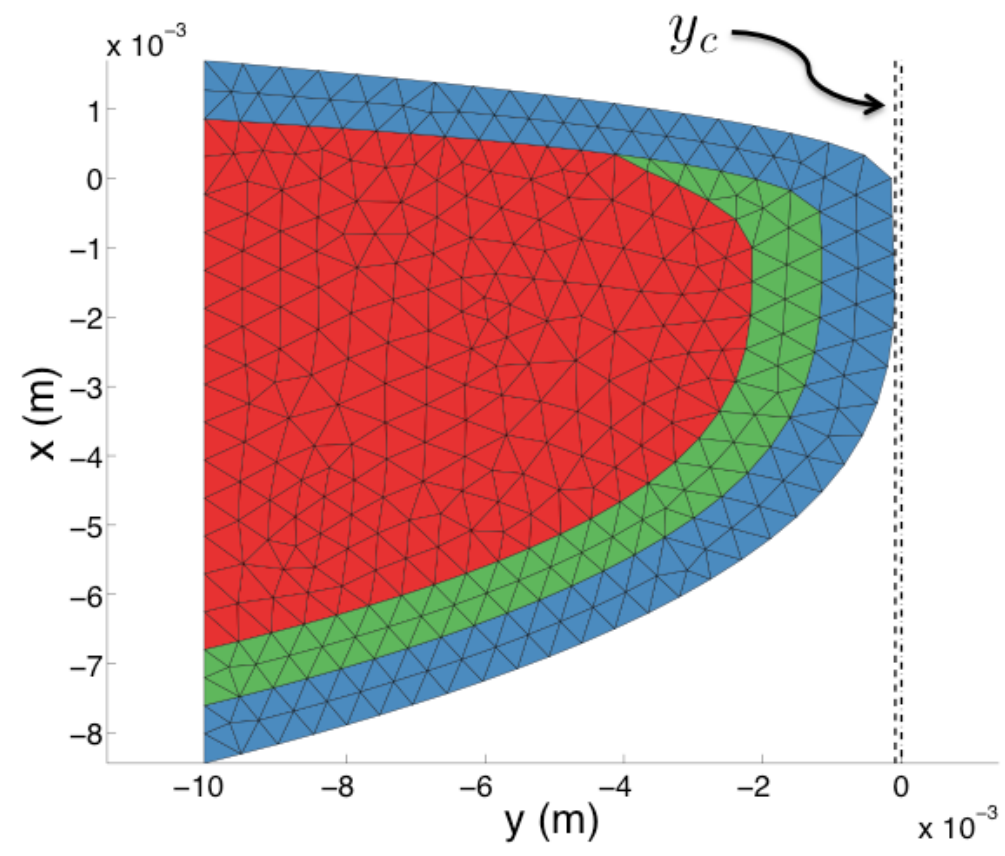

Figure 2.10: The contact line is set at $y_{c}=0.08876 \mathrm{~mm}$ from the medial surface of the vocal fold. The initial gap of the glottis is $0.01 \mathrm{~cm}$.

contact line may be possible to implement in such a piece of software, it would require significant effort to work around the software limitations. Additionally, forcing is generated using Bernoulli flow, which will be discussed in detail in Chapter 3. This method is used for a specific flow regime, and consequently, is not supported in any commercial package. Although commercial packages may facilitate faster development, support for these two conditions is not possible without in-house techniques.

The finite element structural solver was developed in Fortran 90. The flowchart of the implementation is illustrated in Fig. 2.11. The software reads a plaintext input file, which contains information regarding the number of jobs to be run, and the configuration of each job. For each job, meshes are read from specified files, boundary nodes are specified, and the solution is computed for Eq.2.8 and/or Eq. 2.5. Output files are written in plaintext or binary format, and post-processing is performed in Matlab.

The selection of Fortran for development is based on its syntax, its high performance 
capabilities, and its ease of implementation of parallel processing. The syntax of Fortran allows a developer to manipulate individual elements, rows, or columns of matrices without the use of do- or while-loops. Ultimately this has the effect of minimizing the instances of array indexing, which is an expensive operation. Fortran is also a relatively low level language, and consequently, operations run faster than in high level or interpreted languages, such as Matlab. Parallel processing for loops and operations in Fortran are relatively easy to implement, requiring minimal changes to single thread implementations. Although the work presented in this thesis does not make use of parallel processing, it is a recommendation for future use of this work.

\subsection{Verification and Validation}

To ensure that the finite element code performs correctly, two test cases were run and compared to literature solutions. These cases are:

1. free vibration of the three-dimensional vocal fold model, and

2. forced in-plane vibration of a cantilever beam subject to a linearly-varying distributed load.

Running these cases and comparing the solutions will verify that the forced vibration and free vibration solvers converge with increasing spatial and temporal refinement (verification), and that the converged solutions agree with analytical or existing solutions (validation). 


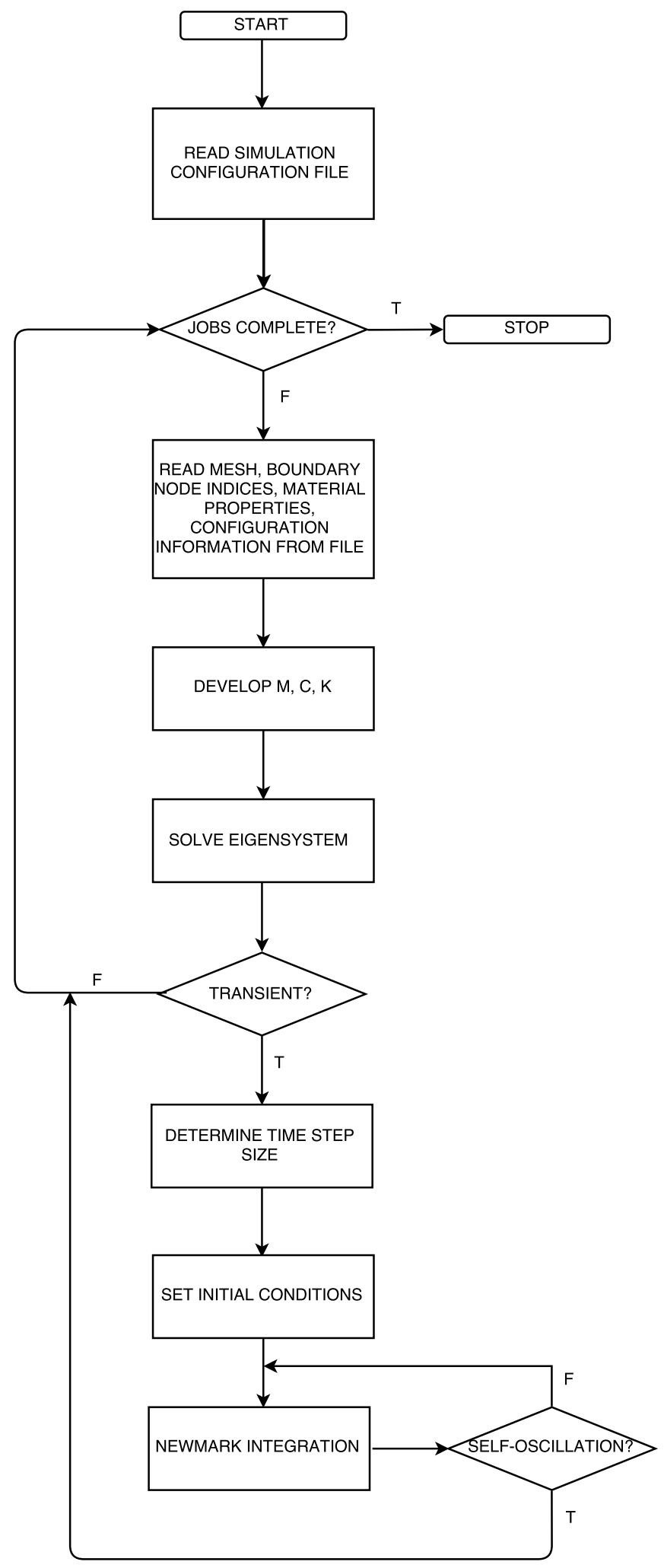

Figure 2.11: Structural solver software flowchart. 


\subsubsection{Free vibration of a three-dimensional vocal fold}

The natural frequencies and modes of vibration of the three-dimensional vocal fold model presented in Section 2.2 were computed for the first four modes. The convergence behaviour of the model is illustrated in Fig. 2.12, which illustrates that the model approaches distinct solutions with mesh refinement. Mesh refinement is expressed in terms of the number of degrees of freedom of the model, which corresponds with three translational degrees of freedom per node (i.e. displacement in the $x, y$, and $z$ directions). Table 2.4 summarizes the first four values of the computed natural frequencies, and shows a maximum percent difference of $1.5 \%$ compared to results in [4]. Differences are attributed to a combination of both numerical error, as well as the fact that the values presented in [4] are also generated via numerical methods.

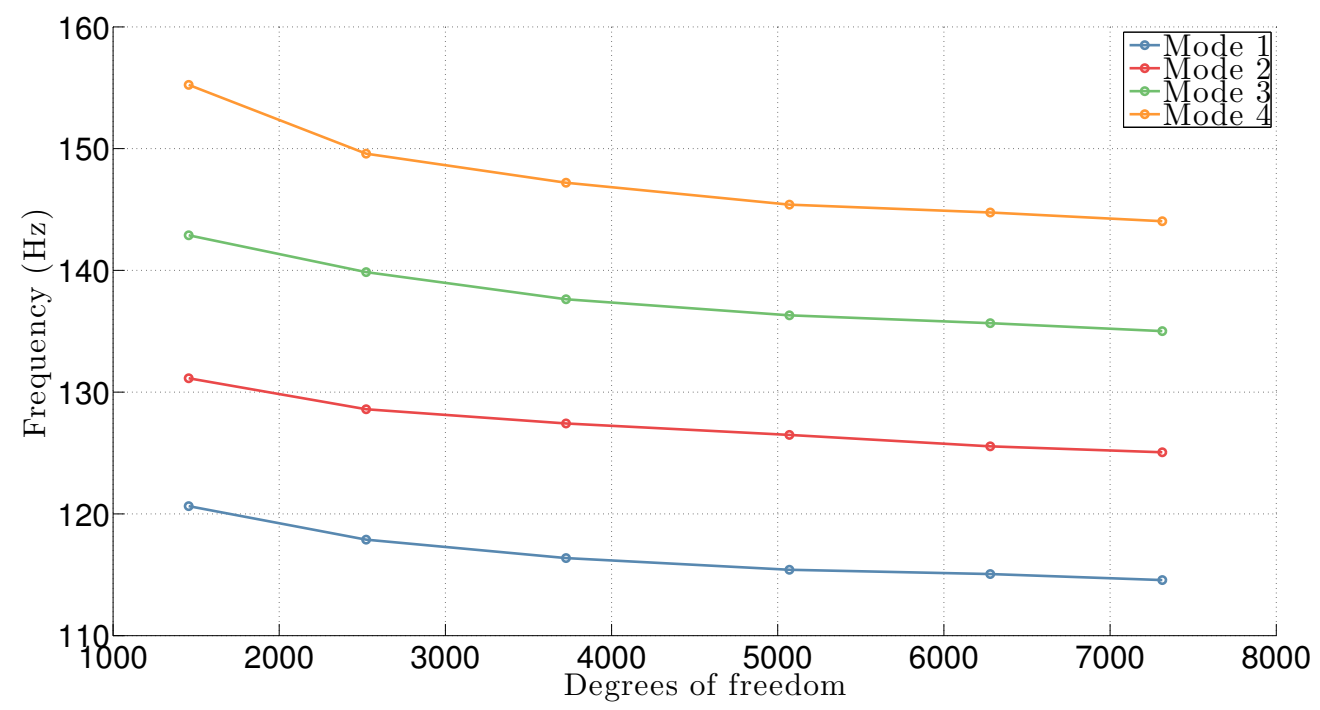

Figure 2.12: Convergence of the transversely isotropic vocal fold model.

The mode shapes of the vocal fold model are also computed, and are illustrated for 
Table 2.4: Comparison of computed natural frequencies of the transversely isotropic vocal fold for the mesh with 7314 degrees of freedom

\begin{tabular}{|l|l|l|l|}
\hline Mode number & Computed $\omega_{i}$ & Literature $\omega_{i}[\mathbf{4}]$ & Percent difference (\%) \\
\hline 1 & 115 & 114 & 0.494 \\
\hline 2 & 125 & 125 & 0.051 \\
\hline 3 & 135 & 133 & 1.50 \\
\hline 4 & 144 & 144 & 0.026 \\
\hline
\end{tabular}

the first four modes in Fig. 2.13(a)-(d). These figures show the periphery of the midcoronal plane of the vocal fold. Solid and dashed lines represent the positive and negative eigenvector mode shapes respectively. Qualitatively, the mode shapes computed in this study are in agreement with those computed in [4]. Note that the mode shapes are computed for one vocal fold only. To compare against the literature model [4], the mode shapes for the left vocal fold are mirrored to illustrate a pair of vocal folds. There is some variation between the computed solutions, which is attributed to the fact that two numerical solutions are being compared, for which there will inevitably be some numerical error. Additionally, this numerical error, as well as the fact that numerical data for these mode shapes has not been published, make quantitative comparison ill advised.
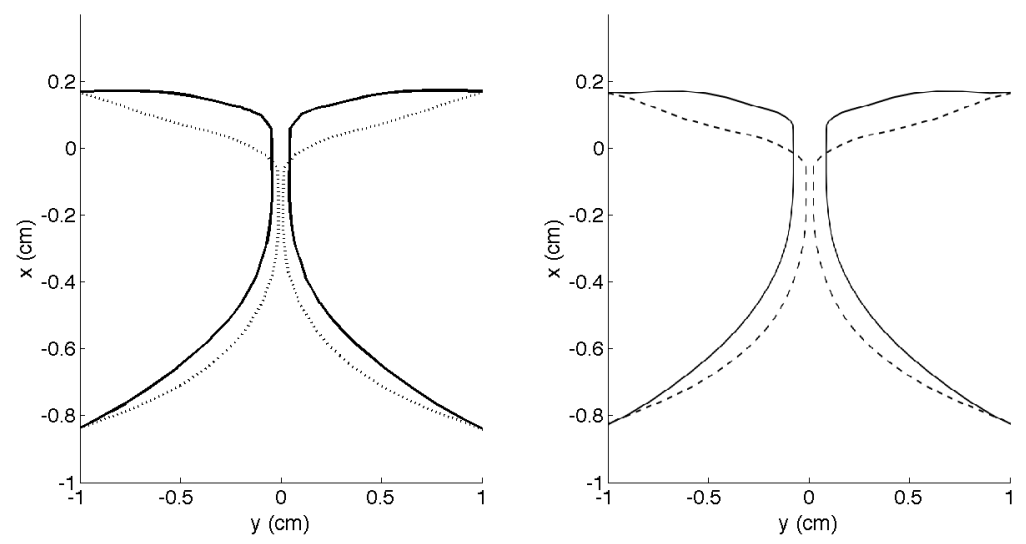

Figure 2.13: (a) Mode 1. Left: Computed solution. Right: Solution from literature [4|. 

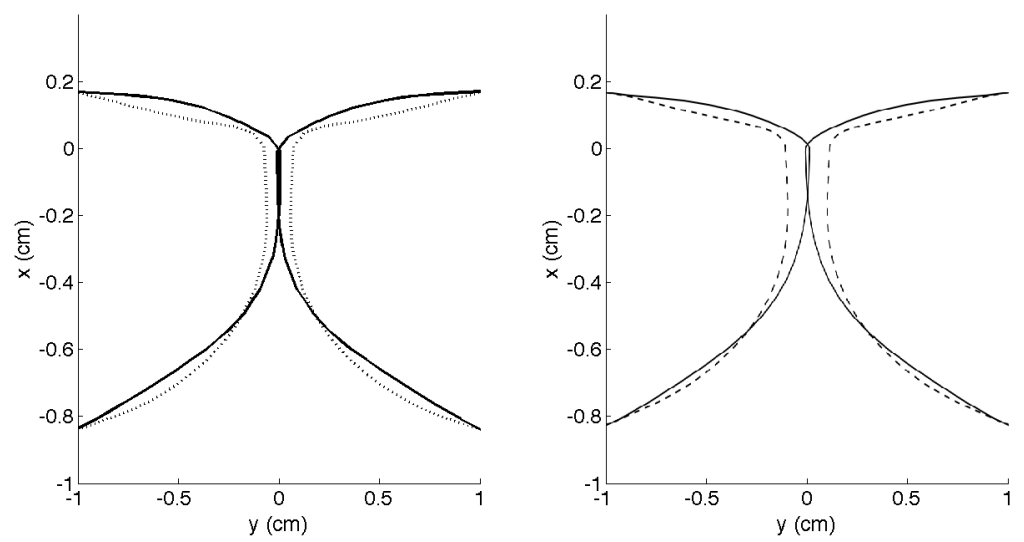

Figure 2.13: (b) Mode 2. Left: Computed solution. Right: Solution from literature [4].
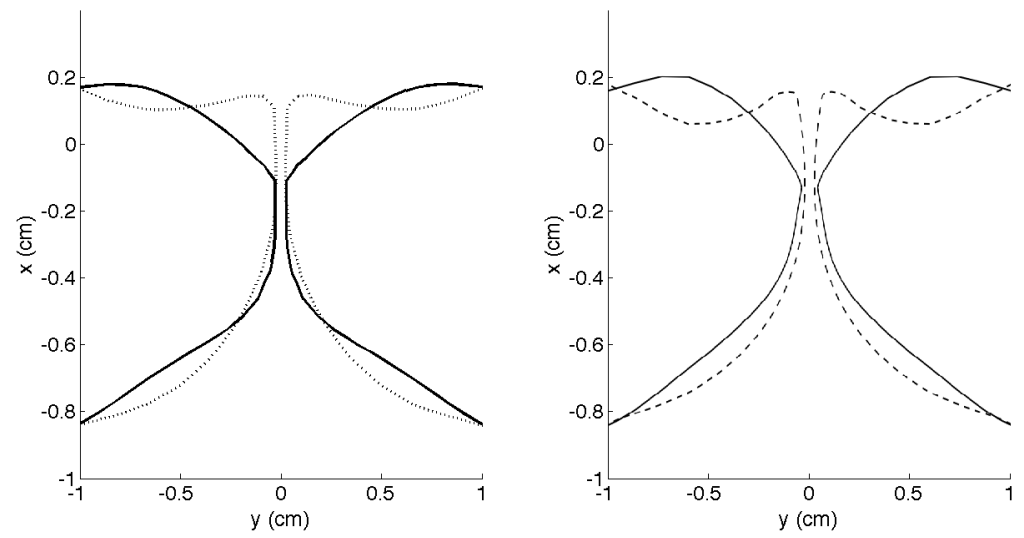

Figure 2.13: (c) Mode 3. Left: Computed solution. Right: Solution from literature 4 .

\subsubsection{Forced vibration of a cantilever beam}

The vibration of a cantilever beam was selected as a verification case because there exist exact solutions for its natural frequencies and maximum static deflection. The first three natural frequencies of a cantilever beam which is fixed at one end and free at the other 

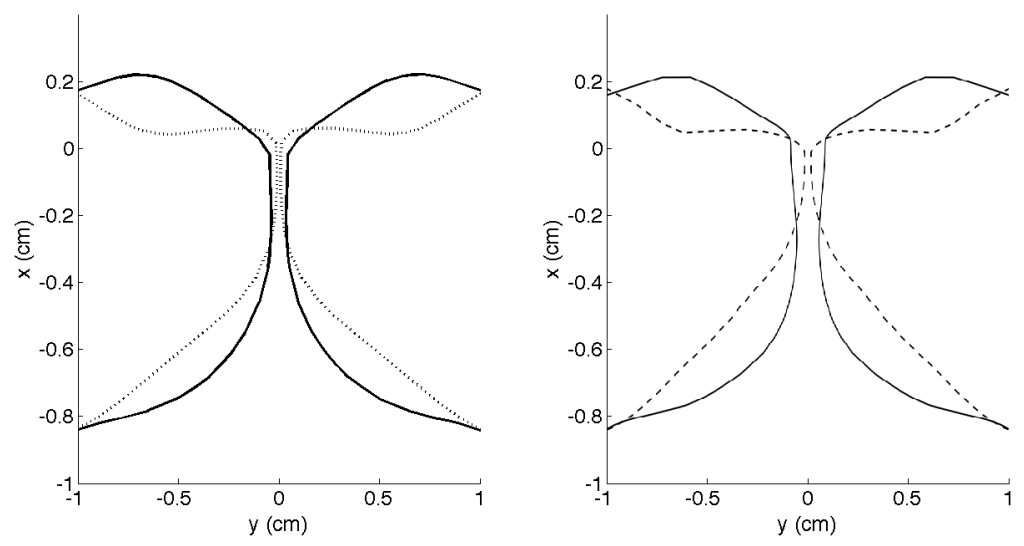

Figure 2.13: (d) Mode 4. Left: Computed solution. Right: Solution from literature [4|.

other is given by

$$
\begin{aligned}
& \omega_{1}=1.875^{2} \sqrt{\frac{E I}{\rho A L^{4}}} \\
& \omega_{2}=4.694^{2} \sqrt{\frac{E I}{\rho A L^{4}}} \\
& \omega_{3}=7.855^{2} \sqrt{\frac{E I}{\rho A L^{4}}},
\end{aligned}
$$

where $I$ is the second moment of area relative to the longitudinal axis, $A$ is the beam's cross-sectional area, and $L$ is the beam's longitudinal dimension [63]. The maximum deflection of a cantilever beam subjected to a linearly varying load along its longitudinal direction is

$$
\delta_{M A X}=\frac{F_{0} L^{4}}{30 E I}
$$

where $F_{0}$ is the peak magnitude of force applied at the fixed end [64, p. 613]. The geometry of the cantilever considered is given in Fig. 2.14, and its corresponding property values are given in Table 2.5. The force is applied as a step at the start of the simulation.

Spatial convergence of the model is illustrated in Fig. 2.15. As the number of degrees of freedom in the model increases, solutions for the first three natural frequencies 


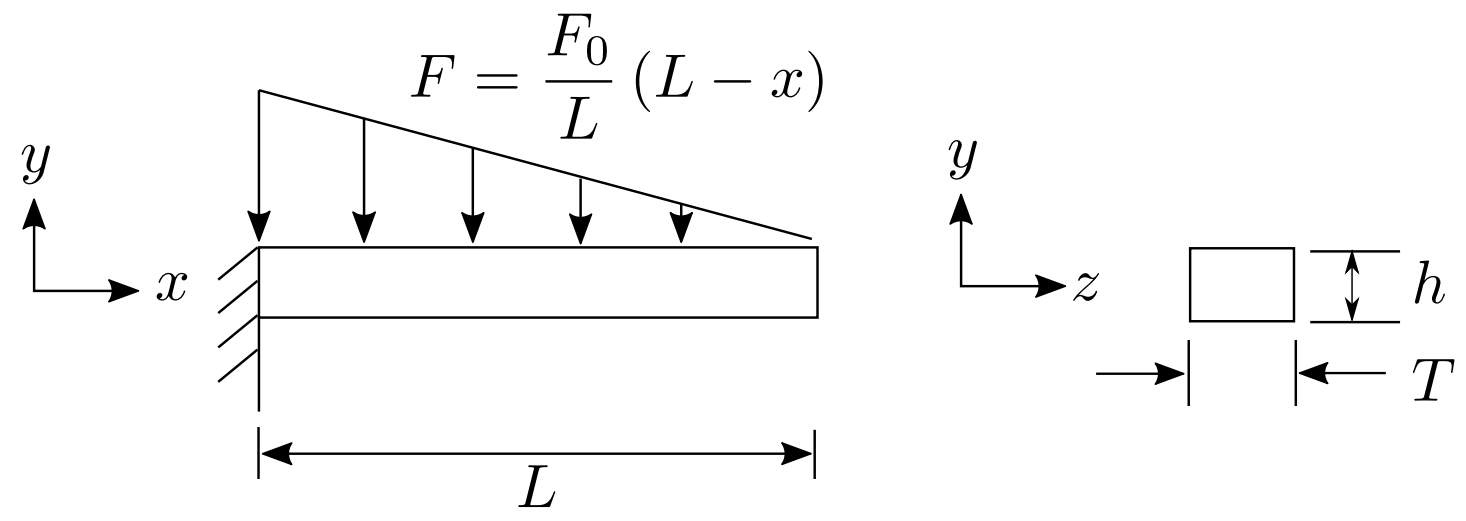

Figure 2.14: Geometry of the loaded cantilever beam

Table 2.5: Numerical properties of the loaded cantilever beam

\begin{tabular}{|l|l|}
\hline Property & Value \\
\hline$L$ & $60.96 \mathrm{~m}$ \\
\hline$h$ & $15.24 \mathrm{~m}$ \\
\hline$T$ & $0.2289 \mathrm{~m}$ \\
\hline$F_{0}$ & $-1000 \mathrm{~N}$ \\
\hline$E$ & $34.474 \times 10^{9} \mathrm{~N} / \mathrm{m}^{2}$ \\
\hline$v$ & 0.11 \\
\hline$\rho$ & $568.2 \mathrm{~kg} / \mathrm{m}^{3}$ \\
\hline
\end{tabular}

is approached. A comparison of these solutions with the exact solution is tabulated in Table 2.6, where a maximum percent error of $5.88 \%$ was found for the third mode. The reason for this discrepancy is due to the exact solution only considering transverse vibration [63], while the finite element model considers both transverse and longitudinal vibration. Although transverse vibration dominates in the lower modes, the influence of longitudinal vibration will increase with successive modes.

Temporal convergence was assessed by solving for the transient motion of the end of the beam in the transverse direction for decreasing time step values. Fig.2.16 illustrates 


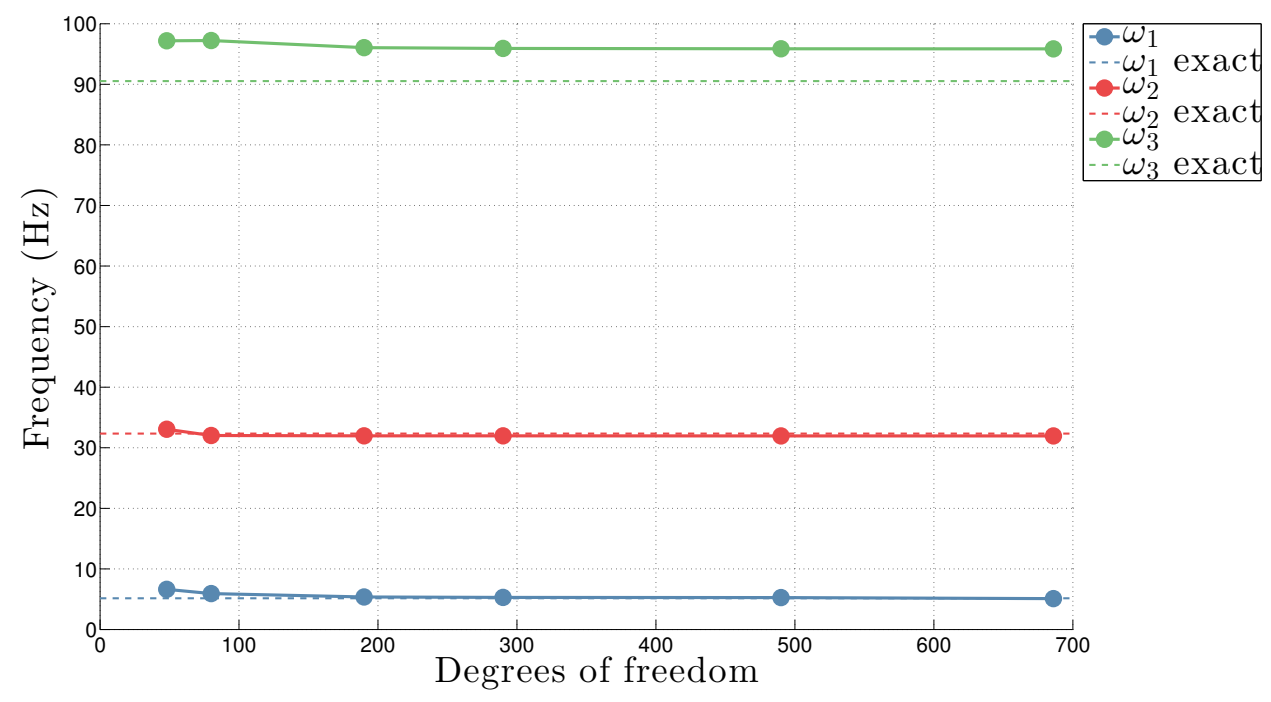

Figure 2.15: Spatial convergence of the cantilever beam.

Table 2.6: Comparison of computed natural frequencies with exact solution for the cantilever beam

\begin{tabular}{|l|l|l|l|}
\hline Mode number & Computed $\omega_{i} \mathbf{( H z )}$ & Exact $\omega_{i} \mathbf{( H z )}$ & Percent error (\%) \\
\hline 1 & 5.10 & 5.16 & 1.08 \\
\hline 2 & 32.0 & 32.3 & 1.17 \\
\hline 3 & 95.9 & 90.5 & 5.88 \\
\hline
\end{tabular}

the behaviour of the model for successively smaller selections of the time step, where $\Delta t_{i}$ corresponds with the period of the $i$ th natural frequency divided by 20 . As the time step decreases, a solution for the periodic vibration of the beam is approached. The exact solution for the static deflection is superimposed on this plot, which was computed as $-0.198 \mathrm{~mm}$, which the beam is shown to vibrate about. The computed percent error for this deflection was found to be $5.77 \%$. 


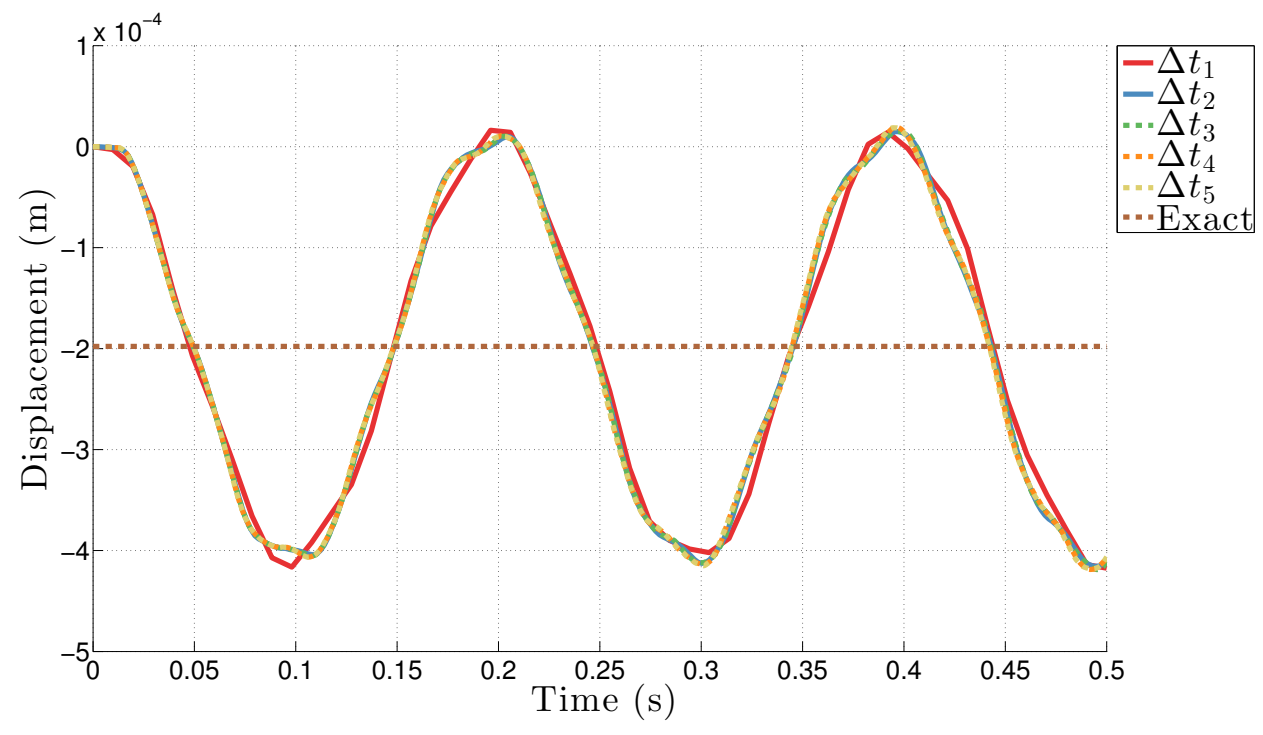

Figure 2.16: Temporal convergence of the cantilever beam. 


\section{Chapter 3}

\section{Aerodynamic Analysis}

\subsection{Introduction}

Modeling of the flow and aerodynamic loading within the larynx is presented in this chapter. A discussion of modeling strategies is given, with emphasis on the balance between computational efficiency and accuracy. Justification of the selection of quasisteady, one-dimensional Bernoulli flow is made, along with an outline of its implementation for computation of the intraglottal pressure distribution. Validation of the model is presented by comparing predicted flow parameters with literature models and experimental measurements.

\subsection{Justification for Bernoulli Flow}

There are primarily two strategies used for resolving the aerodynamics within the larynx in computational vocal fold models: solution of the incompressible Navier-Stokes equations, and solution of the Bernoulli equation. Both strategies possess distinct advantages and disadvantages. Consequently, one strategy will be selected based on the requirements of this study. 
This work seeks to quantify the effect of the additional mass of a polyp on pitchintensity dependence in phonation. To quantify this parameter, time-varying aerodynamic loading conditions must be simulated on the vocal fold surface. Both the Bernoulli equation and the Navier-Stokes equations may predict this loading, however, the Navier-Stokes equations offer considerably better accuracy than the Bernoulli equation. In contrast, the solution of the Bernoulli equation is far more efficient [62].

Navier-Stokes solutions are more accurate due to their resolution of the entire flow field under the influence of viscous, spatial, and temporal effects. By comparison, Bernoulli's equation holds under the assumption of inviscid and steady flow on a one dimensional streamline [22, p. 183]. Literature models which incorporate Bernoulli flow solvers bypass the steady flow assumption by treating the flow as quasi-steady, meaning, the time varying flow may be treated as a series of stationary states as the domain changes in time [65]. This assumption can be made due to the small dimensions of the larynx (where flow behaves as a one-dimensional streamline), and the high velocity of the flow ( $M \approx 0.1$ at the smallest cross-section of the flow during adduction) [30|. The accuracy of the Bernoulli flow model may also be improved by incorporating empirical loss factors to account for the flow of energy resulting from viscous action.

The Navier-Stokes equations offer a disadvantage in that their solution requires considerable overhead and computation time $[62]$. This overhead presents in the form of requiring a method of solving a multi-dimensional set of intricately coupled partial differential equations, and furthermore, a strong two-way coupling between the equations of motion with the Navier-Stokes equations. Multiple methods exist to couple the Lagrangian structural system with the Eulerian fluid system [66], however, this necessitates significant expense in the form of computation time.

Despite the accuracy which is afforded by Navier-Stokes solutions, their limitations 
with regard to computational expense are not suitable for the sensitivity analysis performed in this study. Multiple cases must be run to quantify trends, and accordingly, efficiency is crucial. Furthermore, accuracy in the fluid domain is not of concern in this study, as only the vibration of the vocal folds is considered, as opposed to the resolution of the flow field. The highly efficient Bernoulli equation has demonstrated effective modeling of aerodynamic transfer of energy to the vocal folds through quasi-steady assumptions and the addition of loss factors [62,67]. Therefore, Bernoulli flow is selected as the aerodynamic model for this study.

\subsection{Bernoulli Flow Methodology}

This section outlines the derivation of the pressure distribution in the glottis based on the geometry of the vocal folds, and the constant subglottal pressure (lung pressure) which drives the flow. The Bernoulli equation is invoked under quasi-steady, onedimensional conditions, and is used to compute pressure change between two points in the glottis on a streamline. Volume flow rate is derived by considering a streamline between the stagnant conditions of the lungs and the flow separation point. Empirical coefficients and loss terms are incorporated into these equations to account for flow separation, and losses due to vena contracta in the glottal jet, and pressure loss due to viscous action.

\subsubsection{Computing the pressure distribution}

To derive the pressure distribution within the glottis at time $t$, two arbitrary points along a streamline are defined. The convention which follows is such that point $i$ is located in the direction upstream of point $i+1$. Applying the Bernoulli equation between these 
points,

$$
\frac{P_{i+1}(t)}{\rho}+\frac{\mathbf{u}_{i+1}^{2}(t)}{2}=\frac{P_{i}(t)}{\rho}+\frac{\mathbf{u}_{i}^{2}(t)}{2},
$$

where $P(t)$ is static pressure, $\rho$ is the air density, and $\mathbf{u}(t)$ is the flow velocity [61]. Since information regarding the velocity field is unknown, an expression containing the volume flow rate is substituted. Volume flow rate, $\dot{Q}$, can be expressed as

$$
\begin{aligned}
\dot{Q}(t) & =\mathbf{u}_{i+1}(t) A_{i+1}(t)=\mathbf{u}_{i}(t) A_{i}(t) \\
& \Longrightarrow \quad \mathbf{u}_{i+1}(t)=\frac{\dot{Q}(t)}{A_{i+1}(t)} \\
& \Longrightarrow \quad \mathbf{u}_{i}(t)=\frac{\dot{Q}(t)}{A_{i}(t)}
\end{aligned}
$$

where $A(t)$ is the cross-sectional area of the flow. Eq. 3.2 is substituted into Eq. 3.1 to obtain

$$
\begin{aligned}
& \frac{P_{i+1}(t)}{\rho}+\frac{\dot{Q}^{2}(t)}{2 A_{i+1}^{2}(t)}=\frac{P_{i}(t)}{\rho}+\frac{\dot{Q}^{2}(t)}{2 A_{i}^{2}(t)} \\
& \Rightarrow P_{i+1}(t)=P_{i}(t)+\frac{\dot{Q}^{2}(t)}{2}\left(\frac{1}{A_{i}^{2}(t)}-\frac{1}{A_{i+1}^{2}(t)}\right)
\end{aligned}
$$

Eq. 3.3 may be used to solve for the pressure distribution along a streamline at a stationary point in time [62]. Since subglottal pressure is assumed constant, the pressure may be computed consecutively from point to point in the downstream direction (ex. $P_{1}(t)=$ constant), as illustrated in Fig. 3.1. Eq. 3.3 also requires the cross-sectional area along the streamline, and the volume flow rate. Cross-sectional area may be determined from the nodal coordinates of the finite element model. Volume flow rate, however, must be calculated at every state in time. 


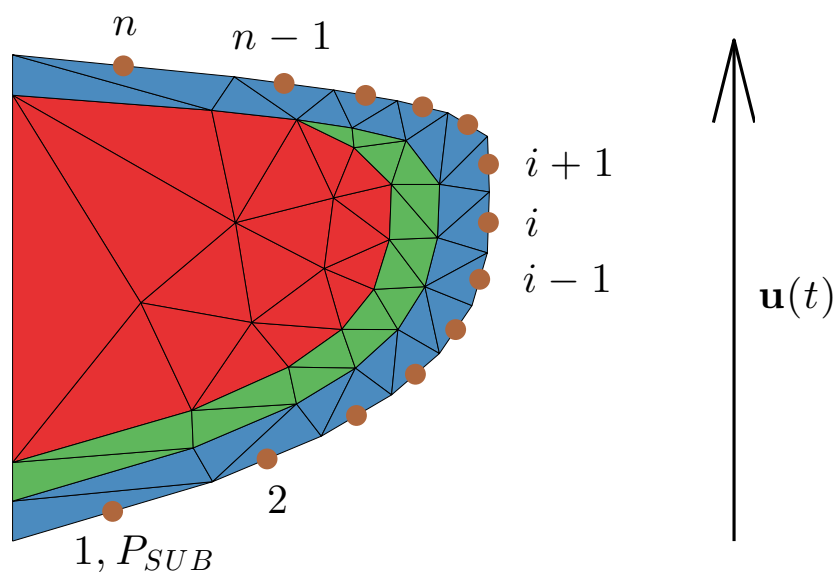

Figure 3.1: Intraglottal pressure is solved along the vocal fold surface in the downstream direction, knowing that subglottal pressure is constant in time.

\subsubsection{Computing the volume flow rate}

The volume flow rate in the larynx at a given time is computed by considering a streamline between the lungs, and the point of flow separation. An illustration of this streamline at time $t$ is shown in Fig. 3.2. Flow properties are related with the Bernoulli equation,

$$
\frac{P_{L}}{\rho}+\frac{\mathbf{u}_{L}^{2}(t)}{2}=\frac{P_{S}(t)}{\rho}+\frac{\mathbf{u}_{S}^{2}(t)}{2}
$$

where subscripts $L$ and $S$ represent lungs, and separation, respectively [68].

At the point of flow separation, static pressure is set equal to the supraglottal pressure, which is zero gauge pressure, i.e. $P_{S}(t) \approx 0$. The lungs are considered a stagnation point along the streamline, where $\mathbf{u}_{L}(t) \approx 0$. Thus, Eq. 3.4 reduces to

$$
\frac{P_{L}}{\rho}=\frac{\mathbf{u}_{S}^{2}(t)}{2} .
$$

To express Eq. 3.5 with respect to volume flow rate, an expression of the form of Eq. 3.2 


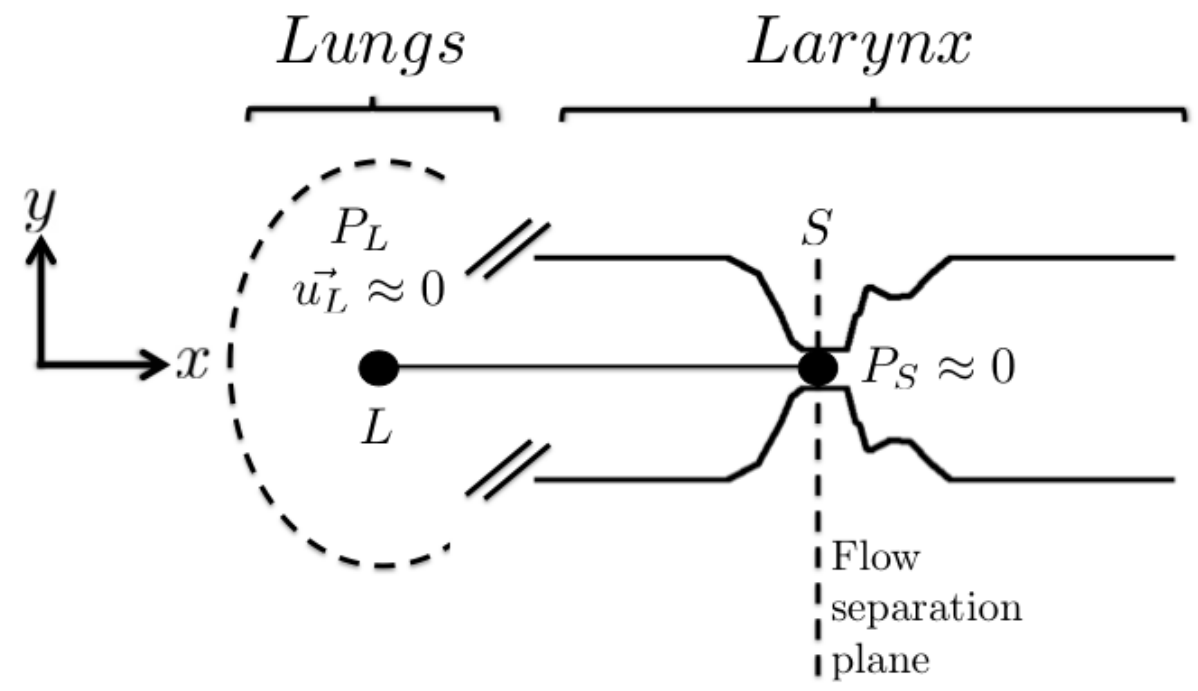

Figure 3.2: Streamline between the lungs and the plane of separation between the vocal folds.

is substituted, yielding

$$
\frac{P_{L}}{\rho}=\frac{\dot{Q}^{2}(t)}{2 A_{S}^{2}(t)} \Rightarrow \dot{Q}(t)=A_{S}(t) \sqrt{\frac{2 P_{L}}{\rho}} .
$$

Eq. 3.6 can thus express the volume flow rate through the larynx as a function of crosssectional area at the separation point. However, the calculation of volume flow rate at a given point of time requires knowledge of the cross-sectional area at the point of flow separation.

\subsubsection{Computing the point of flow separation}

Determination of the point of flow separation is not possible with the Bernoulli equation due to the assumption of inviscid flow. Accordingly, literature models which incorporate Bernoulli flow make use of empirically determined separation area ratios, $\gamma_{S}$, defined as

$$
\gamma_{S}=\frac{A_{S}}{A_{m}},
$$


where $A_{m}$ is the minimum cross-sectional area within the glottis. Values for $\gamma_{S}$ are reported between 1.2-1.47 [62]. For the purposes of this study, it will be assumed that $\gamma_{S}=1.2$

Computational studies have shown that the use of $\gamma_{S}$ results in inaccurate predictions of volume flow rate, but satisfactory prediction of frequencies of vibration [62]. In this study, transient flow simulation will only be performed to quantify the pitchintensity dependency of the vocal folds. Since this dependency requires only accurate computation of the frequencies of vibration, any adverse effects resulting from an improper assumption of $\gamma_{S}$ is mitigated.

\subsubsection{Accounting for energy losses}

The pressure distribution which was derived in Eq. 3.3 does not account for energy losses resulting from viscous action within the larynx. One such effect which must be accounted for is loss associated with vena contracta. Downstream of the glottis, the glottal jet is surrounded by higher pressure stagnant air. This causes the effective area of the jet to decrease. Consequently, the pressure drop across the glottis increases [30]. The loss associated with the vena contracta may be incorporated in Eq. 3.3 by applying a loss factor $\alpha$, such that

$$
P_{i+1}(t)=P_{i}(t)+\frac{\alpha \dot{Q}^{2}(t)}{2}\left(\frac{1}{A_{i}^{2}(t)}-\frac{1}{A_{i+1}^{2}(t)}\right)
$$

Values for alpha have been reported in the range of 1.0-1.5 [30,62]. For the purposes of this study, the midpoint of this range, 1.25, will be assumed as the loss factor.

In addition to the loss associated with the vena contracta, viscous losses due to friction at the walls of the larynx must also be accounted for. The pressure loss associated 
with friction at the walls is given by

$$
\frac{\partial P(t)}{\partial x}=\frac{-12 \mu L^{2} \mathbf{u}(t)}{A^{3}(t)}
$$

where $\mu$ is the dynamic viscosity of the air, and $L$ is the longitudinal distance $x_{i+1}-x_{i}$ [69|. This equation is based on Poiseuille flow, which is derived by assuming fully developed laminar flow between parallel plates. Although the velocity profile in the larynx will not necessarily be fully parabolic, as in Poiseuille flow, experimental studies have shown good agreement between this correction and experimental measurements [69]. Separating variables and integrating Eq. 3.9 , the pressure drop due to wall shear is

$$
\Delta P=\frac{-12 \mu \dot{Q} L}{A^{3}(t)}
$$

Eq. 3.10 is added as a loss term in Eq. 3.8, resulting in

$$
P_{i+1}(t)=P_{i}(t)+\frac{\alpha \dot{Q}^{2}(t)}{2}\left(\frac{1}{A_{i}^{2}(t)}-\frac{1}{A_{i+1}^{2}(t)}\right)-\frac{12 \mu \dot{Q} L}{A^{3}(t)}
$$

Eq. 3.11 may now be used to solve for the intraglottal pressure distribution at a given point in time [62].

\subsection{Computation of Aerodynamic Forces}

To simulate forcing in the equation of motion, nodes in contact with the flow, called "wet nodes", must be subjected to aerodynamic forces. Aerodynamic forces are calculated from the pressure distribution along the vocal fold length. Eq. 3.11 computes the pressure distribution at the center of the line segments of the wet nodes, as shown in Fig. 3.3. A traction force is computed at this position by multiplying the pressure by the 
element thickness, and setting its direction in compression, normal to the segment. This is represented as

$$
\frac{\mathbf{F}}{L}=(P T) \cdot \hat{\mathbf{n}}
$$

where $\mathbf{F}$ is the applied force vector, $L$ is a length vector along the line segment, and $T$ is the element thickness. Based on the orientation of $L$, the traction vector may then be split into component tractions $\mathbf{F}_{x}$ and $\mathbf{F}_{y}$.

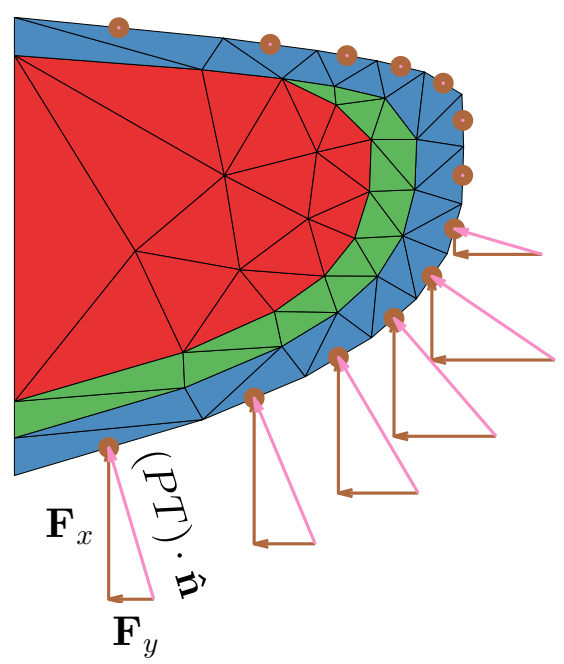

Figure 3.3: Aerodynamic forces are computed at the centroid of each "wet" segment, and split into component forces.

Component tractions are then distributed to the wet nodes through node by node lumping. In node by node lumping, the force contribution of half of each neighboring segment is added to the sandwiched node [70], as shown in Fig. 3.4 .

\subsection{Implementation}

The Bernoulli flow subroutine is called upon in the transient structural solver to return the aerodynamic forces along the vocal fold surface for each time step. The subroutine 


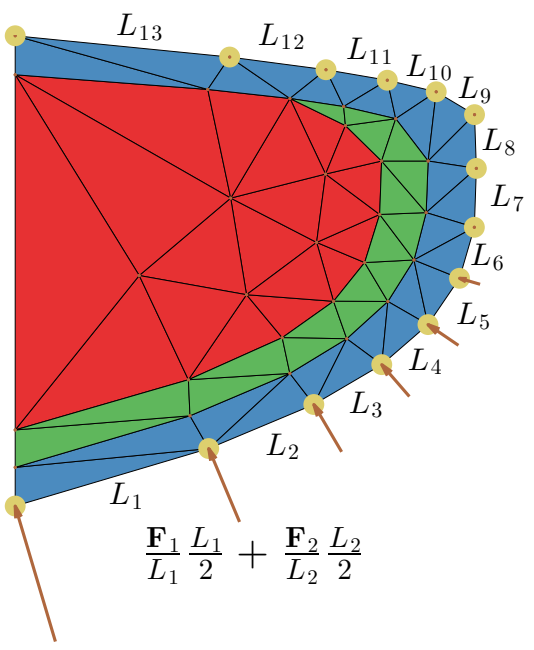

Figure 3.4: Component forces are distributed to the nodes of the mesh via node by node lumping.

runs through the following steps:

1. The cross-sectional area distribution through the larynx is calculated based on the position of nodes on the surface of the vocal fold.

2. Eq. 3.7 is solved for $A_{S}$.

3. Eq. 3.2 is solved for $\dot{Q}$.

4. Eq. 3.11 is solved for the static pressure along the surface of the vocal fold in the downstream direction.

5. Pressure traction components are calculated, and are distributed to wet nodes using node by node lumping.

Following this process, the structural solver computes the motion of the vocal folds for another time time step, and the process repeats. 


\subsection{Validation}

To the author's knowledge, there is no published data for the flow parameters around the selected vocal fold model. Accordingly, flow parameters will be qualitatively compared to similar models and experimental data. Table 3.1 gives information regarding the flow values which are held constant for all comparisons.

Table 3.1: Flow model properties

\begin{tabular}{|l|l|}
\hline Parameter & Value \\
\hline Density, $\rho\left(\mathrm{kg} / \mathrm{m}^{3}\right)$ & 1.2 \\
\hline Dynamic viscosity, $\mu\left(\mathrm{kg} \mathrm{m}^{-1} \mathrm{~s}^{-1}\right)$ & $1.8 \times 10^{-5}$ \\
\hline Vena contracta loss coefficient, $\alpha$ & 1.25 \\
\hline Flow separation ratio, $\gamma_{S}$ & 1.47 \\
\hline Supraglottal pressure, $P_{S}(\mathrm{~Pa}(\mathrm{~g}))$ & 0 \\
\hline
\end{tabular}

Fig. 3.5 compares the pressure distribution about two vocal fold models. One such model, from Decker \& Thomson [62], is similar to the model used in this study, in that it is approximately the same length in both the medial and lateral directions, and by extension, has a similar minimum cross-sectional flow area. However, this model is also highly idealized, as can be seen by the lower part of the vocal fold being modeled as a straight line, unlike the convex geometry used in this study. Decker \& Thomson resolve for the pressure distribution using the Navier-Stokes equations.

Decker \& Thomson assume subglottal pressure of $1 \mathrm{kPa}$, and accordingly, this value is also used for comparison. Both models observe the same rate of pressure drop, however, Decker \& Thomson's model shows that flow separation occurs earlier. This discrepancy is due to the difference in geometry. The vocal fold used in this study, due to its convex shape, has a faster rate of area decrease in the downstream direction than Decker \& 


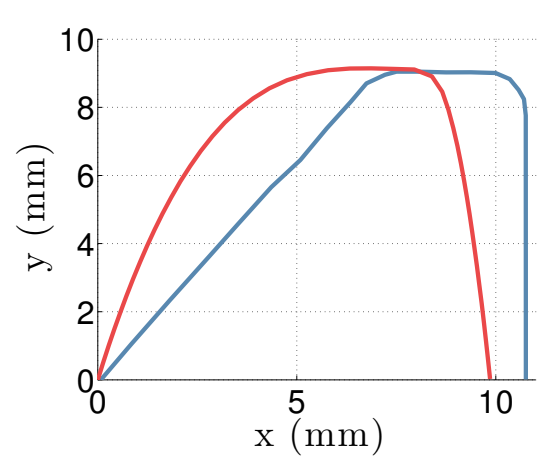

(a) Vocal fold profile comparison.

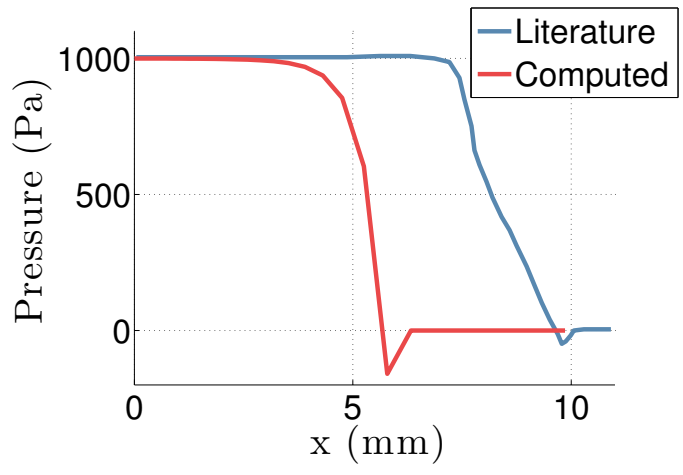

(b) Pressure distribution comparison.

Figure 3.5: Comparison of pressure distribution along separate vocal fold models. Blue: Decker \& Thomson, 2007 [62]. Red: Vocal fold incorporated in this study.

Thomson's model, and consequently, separation position is pushed upstream. Furthermore, a faster rate of area decrease leads to a larger pressure drop in the glottis. Despite these differences, the qualitative trends which would be expected of the flow model are in agreement with theory. Pressure drops along the vocal fold length, and separates following a significant pressure drop in the glottis, where aerodynamic transfer of energy occurs.

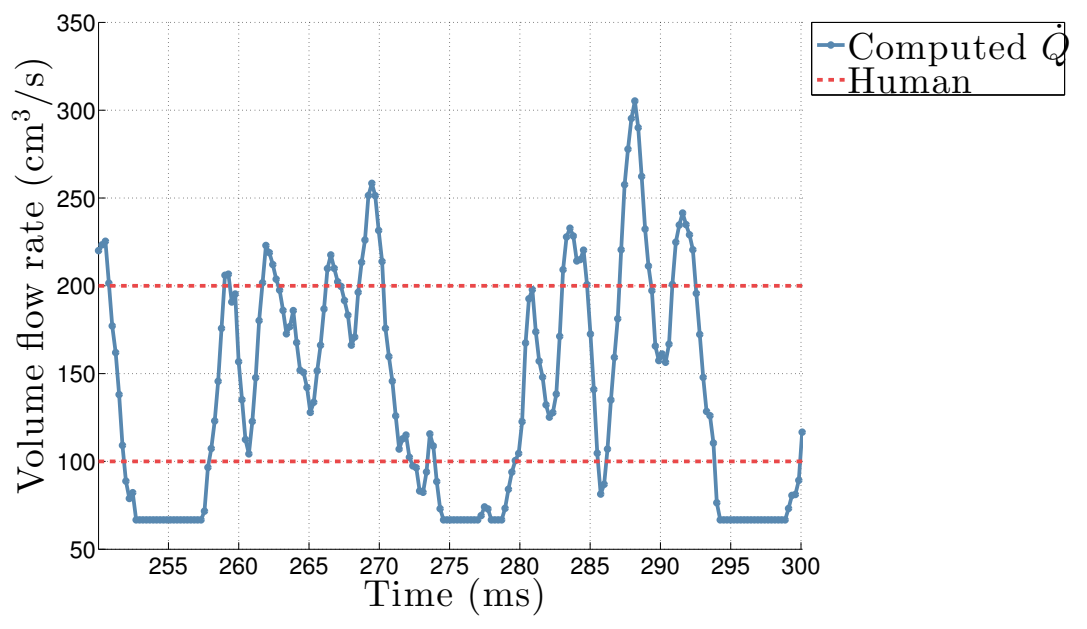

Figure 3.6: Computed flow rate for 200 Pa subglottal pressure. 
Fig. 3.6 shows the computed variation of volume flow rate in time for a subglottal pressure of $200 \mathrm{~Pa}$. This value of subglottal pressure was selected because the corresponding range required for human phonation at a conversational volume is $200 \mathrm{~Pa}-$ $300 \mathrm{~Pa}[4]$. This plot illustrates a cyclic variation in volume flow rate whose frequency corresponds with the vocal fold's mucosal wave propagation. When the volume flow rate is at its minimum, the vocal folds are near contact.

Fig. 3.6 also shows the ranges for volume flow rate measured for human larynges [71]. The computed volume flow rate falls within this range. Pressure which falls outside of this range may be due to idealized geometric modeling, or the incorporation of a subglottal pressure which was higher than that used to measure the human range.

Fig. 3.7 shows two images of the vocal folds during the abduction and adduction phase, respectively. These images were obtained for a simulation with a subglottal pressure of $300 \mathrm{~Pa}$. The vocal folds show the behaviour which would be expected during the phonation cycle. The medial surface of the vocal fold approaches the centerline during abduction, and pulls away during adduction.

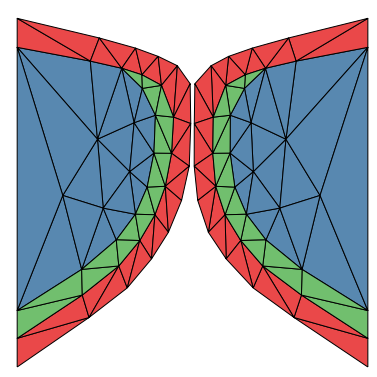

(a) Snapshot of abduction.

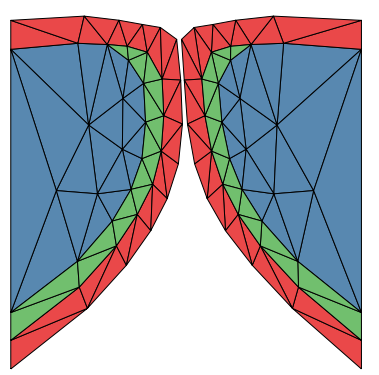

(b) Snapshot of adduction.

Figure 3.7: Snapshot of the vocal folds at abduction and adduction.

A more in-depth view of the mucosal wave which travels along the medial surface 
can be viewed in Fig. 3.8. This figure traces the $x$ displacement of a node at the top of the medial surface of the vocal fold with time. The fundamental frequency of this motion is the dominating periodic motion. The peaks which can be seen that populate the curve represent mucosal wave motion which moves along the surface throughout the cycle. Given that the computed vocal fold vibration displays the expected physiological trends, the aerodynamic model which forces the model is considered to be adequate for further simulations with the pathological model.

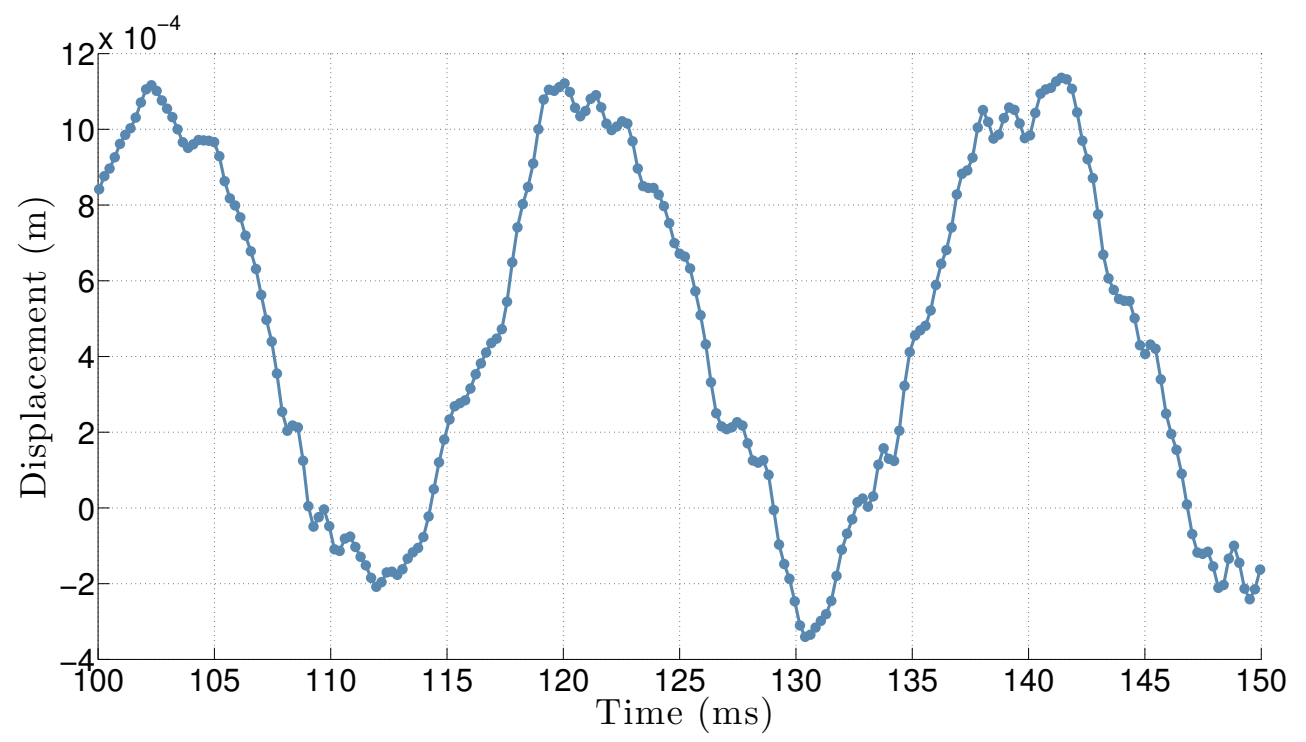

Figure 3.8: Time-history of displacement of a point on the vocal fold surface near the top edge of the medial plane. 


\section{Chapter 4}

\section{Results and Discussion}

\subsection{Introduction}

This chapter presents the results of a three-dimensional sensitivity analysis of polyp parameters, and a two-dimensional investigation of the influence of polyps on pitchintensity dependence.

The sensitivity analysis is performed in the frequency domain with the threedimensional model. The continuous parameters of the polyp are divided into four distinct states each, within their physiological limits. Spatial convergence is quantified for 16 sample cases based on the extremes of these interactions using Taguchi's orthogonal array method [72]. The interactions between mass and stiffness, and mass and position are then presented as a function of the change in natural frequency relative to a baseline vocal fold without a polyp. The effect of varying the polyp's aspect ratios are presented with respect to its natural frequencies.

Pitch-intensity dependence is assessed using two-dimensional models of vocal fold pairs, each containing a healthy vocal fold, and a vocal fold with a polyp. These vocal folds are aerodynamically loaded in time. Two sets of simulations are run for two different sizes of polyps. Each set of simulations varies the subglottal pressure such that a 
linear trend is inferred between pitch and intensity.

\subsection{Sensitivity Analysis of Polyp Parameters}

The sensitivity of the three-dimensional polyp model will be studied based on examining how changes to its properties affect the natural frequencies of a vocal fold with this polyp. To perform this analysis, the continuous polyp variables need to be discretized to a finite number of states. The proposed states of the model's parameters are split equally into four distinct values, each of which is given in Table 4.1 .

Table 4.1: Polyp parameters and nominal state values

\begin{tabular}{|l|l|l|l|l|}
\hline \multirow{2}{*}{ Parameter } & \multicolumn{4}{l|}{ State } \\
\cline { 2 - 5 } & $\mathbf{1}$ & $\mathbf{2}$ & $\mathbf{3}$ & $\mathbf{4}$ \\
\hline $\mathbf{A}$ & $0.5 \mathrm{~mm}$ & $1.5 \mathrm{~mm}$ & $2.5 \mathrm{~mm}$ & $3.5 \mathrm{~mm}$ \\
$\mathbf{B}$ & $0.5 \mathrm{~mm}$ & $1.5 \mathrm{~mm}$ & $2.5 \mathrm{~mm}$ & $3.5 \mathrm{~mm}$ \\
$\mathbf{C}$ & $0.5 \mathrm{~mm}$ & $1.5 \mathrm{~mm}$ & $2.5 \mathrm{~mm}$ & $3.5 \mathrm{~mm}$ \\
$\mathbf{C}$ & $0 \mathrm{~mm}$ & $1.17 \mathrm{~mm}$ & $2.3 \mathrm{~mm}$ & $3.5 \mathrm{~mm}$ \\
$\alpha_{\mathbf{P}}$ & 2 & 3 & 4 & 5 \\
\hline
\end{tabular}

\subsubsection{Spatial convergence analysis}

The interaction studies require several cases to be run to infer trends. To be confident in the computed trends, each three-dimensional mesh should be run until convergence, as was performed in Section 2.7 with the validation cases. Rather than perform a convergence analysis for every possible mesh (1024 total runs for five parameters with four states each), a sample of meshes will be run to convergence. This sample will represent the minimum number of cases which sample the extremes of all possible parameter interactions. The maximum number of degrees of freedom required for convergence will 
be used as the metric for remaining simulations.

Taguchi's orthogonal array (OA) method is used in experimental analysis to assess the sensitivity of experimental parameters. The OA method offers a way of sampling from this large set of configurations such that one may examine all pairings of states at least once [72]. This reduces the set of cases from 1024 runs to 16 runs. The OA for this spatial convergence analysis is given in Table 4.2. The rightmost column contains the number of degrees of freedom required for the model to reach convergence. Variation in this value is due to the necessary refinement required for various configurations of the polyps.

Table 4.2: Taguchi L16 OA for polyp parameter states

\begin{tabular}{|l|l|l|l|l|l|l|}
\hline Experiment & A & B & C & $\mathbf{C}$ & $\alpha_{\mathbf{P}}$ & DOF \\
\hline 1 & 1 & 1 & 1 & 1 & 1 & 6357 \\
\hline 2 & 1 & 2 & 2 & 2 & 2 & 6417 \\
\hline 3 & 1 & 3 & 3 & 3 & 3 & 5337 \\
\hline 4 & 1 & 4 & 4 & 4 & 4 & 4503 \\
\hline 5 & 2 & 1 & 2 & 3 & 4 & 4263 \\
\hline 6 & 2 & 2 & 1 & 4 & 3 & 4314 \\
\hline 7 & 2 & 3 & 4 & 1 & 2 & 5316 \\
\hline 8 & 2 & 4 & 3 & 2 & 1 & 4365 \\
\hline 9 & 3 & 1 & 3 & 4 & 2 & 4335 \\
\hline 10 & 3 & 2 & 4 & 3 & 1 & 4395 \\
\hline 11 & 3 & 3 & 1 & 2 & 4 & 4398 \\
\hline 12 & 3 & 4 & 2 & 1 & 3 & 3921 \\
\hline 13 & 4 & 1 & 4 & 2 & 3 & 4416 \\
\hline 14 & 4 & 2 & 3 & 1 & 4 & 3915 \\
\hline 15 & 4 & 3 & 2 & 4 & 1 & 4485 \\
\hline 16 & 4 & 4 & 1 & 3 & 2 & 4449 \\
\hline
\end{tabular}


Table 4.2 shows that the largest number of degrees of freedom required was for experiment 2, which required 6417 degrees of freedom to reach convergence. Accordingly, the numerical experiments to follow will all be run with meshes which more than 6417 degrees of freedom to circumvent the requirement for numerous convergence analyses.

\subsubsection{Interaction of mass and stiffness}

To investigate the interaction between a polyp's mass and stiffness, a set of threedimensional simulations is run which isolates these parameters. The polyp's shape is held as spherical $(A=B=C)$, and the position is fixed at $x=0 \mathrm{~mm}$. Mass is increased for each sequential set of simulations by incrementing the state of $A, B$, and $C$ from one to four. Each of these four sets of simulations is run four times for each stiffness value. A visualization of this simulation set-up is illustrated in Fig. 4.1 and the states of each run and first four natural frequencies are tabulated in Table 4.3 .
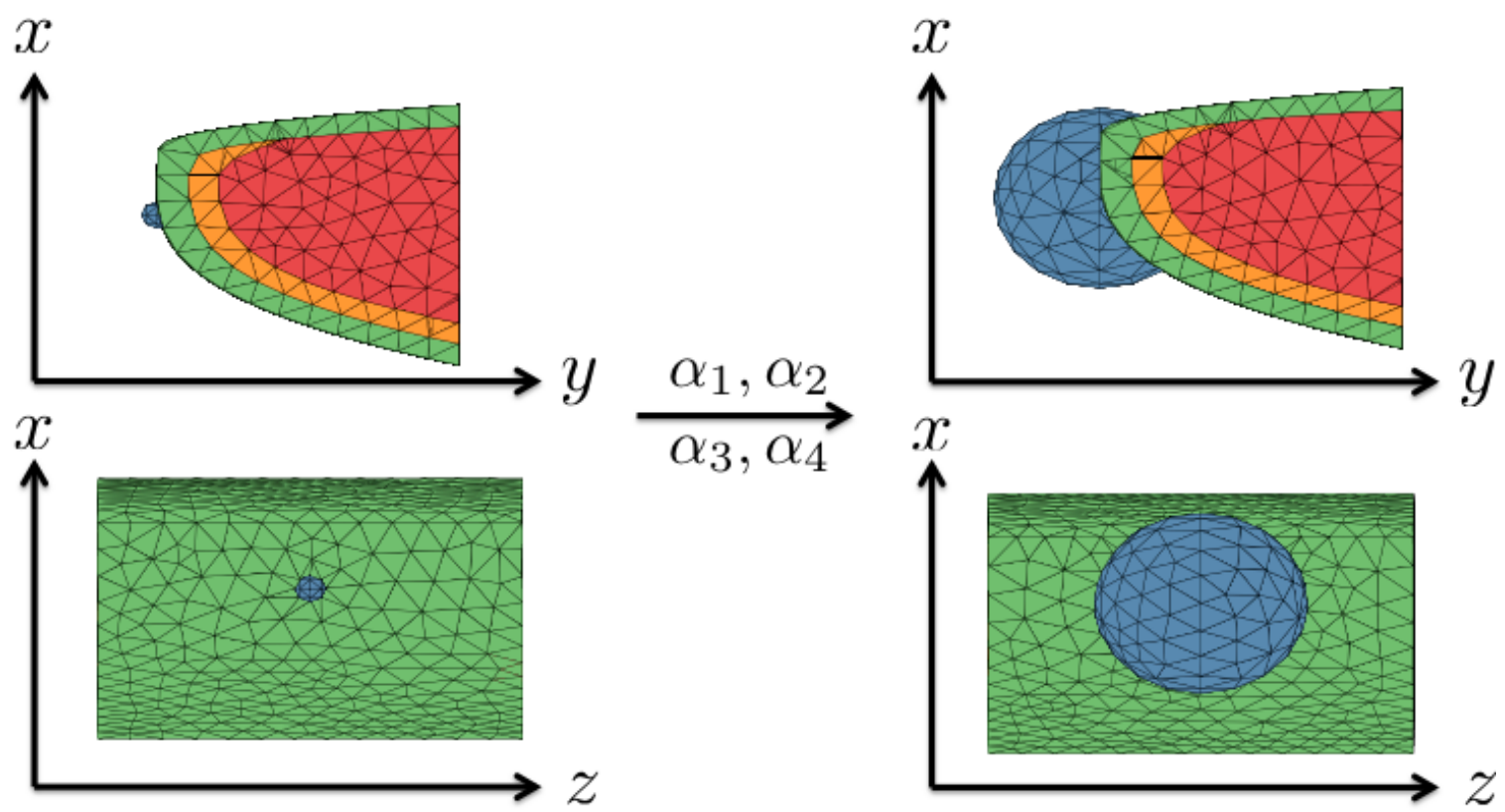

Figure 4.1: Simulation set-up for examination of the interaction of mass and stiffness. The mass and stiffness in the three-dimensional mesh is varied for multiple cases. 
Table 4.3: Mass and stiffness interaction simulation set-up, and first four computed natural frequencies

\begin{tabular}{|l|l|l|l|l|l|l|l|l|l|}
\hline \multirow{2}{*}{ Run } & \multicolumn{9}{|l|}{ State } \\
\cline { 2 - 10 } & $\mathbf{A}$ & $\mathbf{B}$ & $\mathbf{C}$ & $\mathbf{C}$ & $\alpha_{\mathbf{P}}$ & $\omega_{\mathbf{1}}$ & $\omega_{\mathbf{2}}$ & $\omega_{\mathbf{3}}$ & $\omega_{\mathbf{4}}$ \\
\hline 1 & 1 & 1 & 1 & 1 & 1 & 115.34 & 126.29 & 136.13 & 145.26 \\
\hline 2 & 2 & 2 & 2 & 1 & 1 & 113.25 & 124.11 & 127.85 & 144.92 \\
\hline 3 & 3 & 3 & 3 & 1 & 1 & 85.911 & 108.89 & 119.47 & 142.00 \\
\hline 4 & 4 & 4 & 4 & 1 & 1 & 62.031 & 94.300 & 115.32 & 123.12 \\
\hline 5 & 1 & 1 & 1 & 1 & 2 & 115.35 & 126.30 & 136.13 & 145.26 \\
\hline 6 & 2 & 2 & 2 & 1 & 2 & 113.31 & 124.22 & 127.90 & 144.94 \\
\hline 7 & 3 & 3 & 3 & 1 & 2 & 86.095 & 109.21 & 119.53 & 142.18 \\
\hline 8 & 4 & 4 & 4 & 1 & 2 & 62.240 & 94.726 & 115.59 & 124.33 \\
\hline 9 & 1 & 1 & 1 & 1 & 3 & 115.35 & 126.31 & 136.13 & 145.27 \\
\hline 10 & 2 & 2 & 2 & 1 & 3 & 113.34 & 124.28 & 127.93 & 144.95 \\
\hline 11 & 3 & 3 & 3 & 1 & 3 & 86.190 & 109.39 & 119.57 & 142.27 \\
\hline 12 & 4 & 4 & 4 & 1 & 3 & 62.348 & 94.961 & 115.73 & 125.01 \\
\hline 13 & 1 & 1 & 1 & 1 & 4 & 115.35 & 126.31 & 136.14 & 145.27 \\
\hline 14 & 2 & 2 & 2 & 1 & 4 & 113.36 & 124.32 & 127.95 & 144.95 \\
\hline 15 & 3 & 3 & 3 & 1 & 4 & 86.248 & 109.50 & 119.59 & 142.33 \\
\hline 16 & 4 & 4 & 4 & 1 & 4 & 62.414 & 95.110 & 115.82 & 125.45 \\
\hline
\end{tabular}

Two trends may be inferred from these results. An increase in mass results in a decrease in natural frequency, and an increase in stiffness results in an increase in natural frequency. A contour plot illustrating these trends is given for the first mode in Fig. 4.2 . Contour plots for the higher modes are given in Appendix B. These contours illustrate that the influence of mass is considerably greater than the influence of stiffness.

The mass of the polyp is the dominating factor. The excess mass of the polyp results in damping of the surrounding tissue, resulting in a sharp decrease in natural frequency 


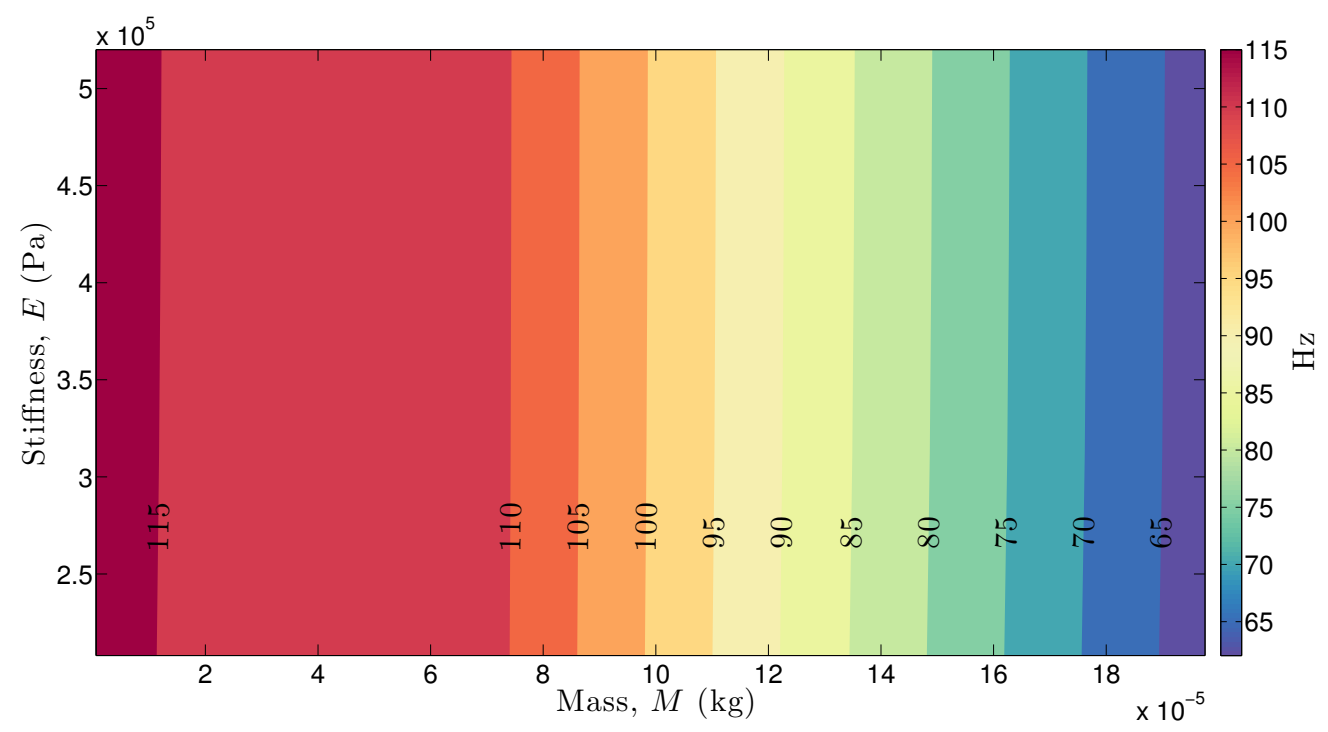

Figure 4.2: Contour plot of fundamental frequency as a function of mass and stiffness.

with increasing mass. Stiffness has almost negligible influence on the natural frequencies of the model. The polyp's stiffness is already at least eight times larger than the surrounding mucosal tissue, so consequently, increases in stiffness yield minimal effects. It would be expected that even larger increases in stiffness would not present with significant changes in the result.

To quantify the influence of stiffness on fundamental frequency, Fig. 4.3 illustrates changes in natural frequency as a function of mass for stiffness $E_{1}$ (i.e. $E_{1}$ is the stiffness of a polyp with $\alpha_{1}$ ), and the changes in natural frequency as a function of stiffness for $M_{1}$ (i.e. $M_{1}$ is the mass of a polyp with $A=B=C=1$ ). For the state $M_{1}, E_{1}$, the computed natural frequency is larger than the baseline natural frequency. This indicates that for small polyp mass, the influence of stiffness is actually greater than the influence of mass. However, for all masses greater than $M_{1}$, the influence of mass dominates, as the computed natural frequencies drop significantly with $M$. These trends hold true for the first four modes. Higher mode influence plots are found in Appendix B.

The results of this numerical experiment indicate that the manifestation of a polyp 

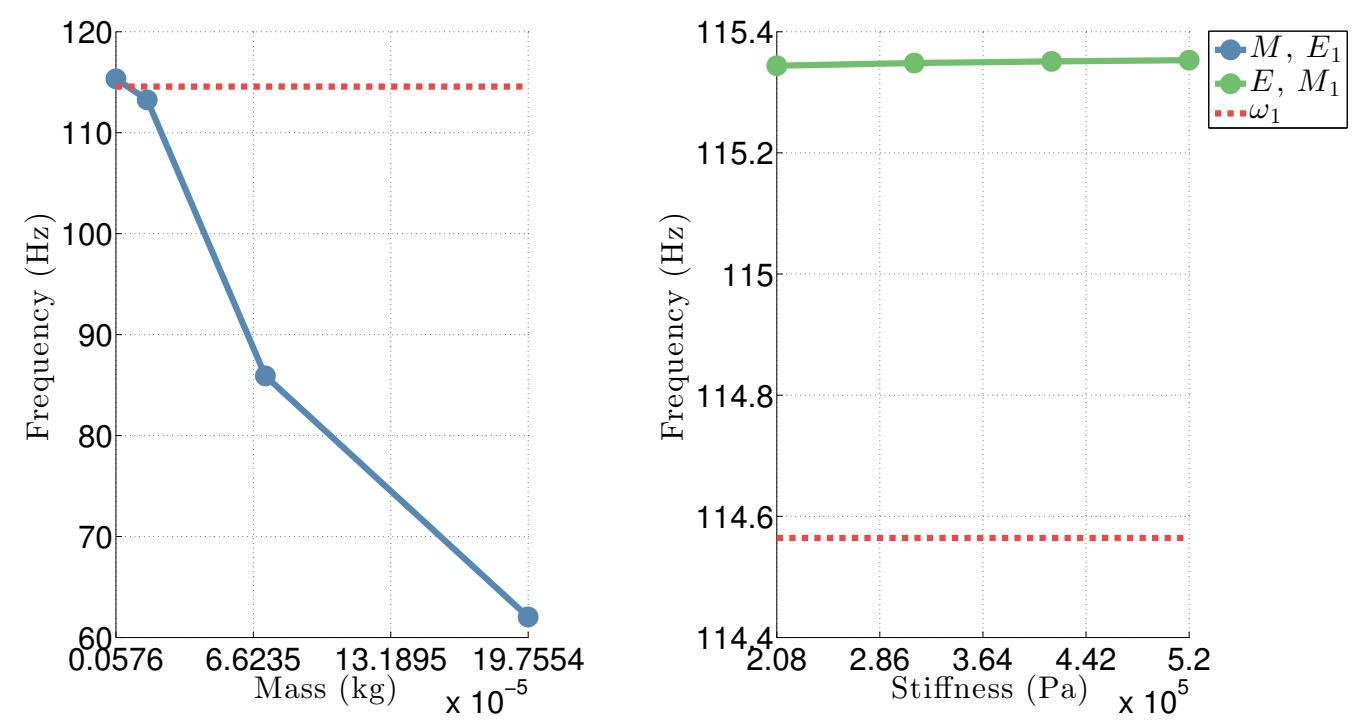

Figure 4.3: Relative influence of mass and stiffness on fundamental frequency. Left: $\omega_{1}$ as a function of mass for $E_{1}$. Right: $\omega_{1}$ as a function of stiffness for $M_{1}$.

on a vocal fold will likely lead to a decrease in pitch. Since a major factor in determining pitch is fundamental frequency [45], decrease in pitch is proportional to polyp mass. By the same token, it is possible to observe an increase in pitch for highly stiff, but small polyp masses.

\subsubsection{Interaction of mass and position}

Similar to the previous analysis, the interaction between a polyp's mass and anteriorposterior position is examined by running a set of three-dimensional simulations which isolates these parameters. The polyp's shape is held spherical, and the stiffness value remains at $\alpha_{P}=2$. Mass is increased for each sequential set of simulations, and run for each state of $c$. An illustration of the simulation set-up is given in Fig. 4.4, and the states for each run are tabulated in Table 4.4, along with the first four computed natural frequencies. 

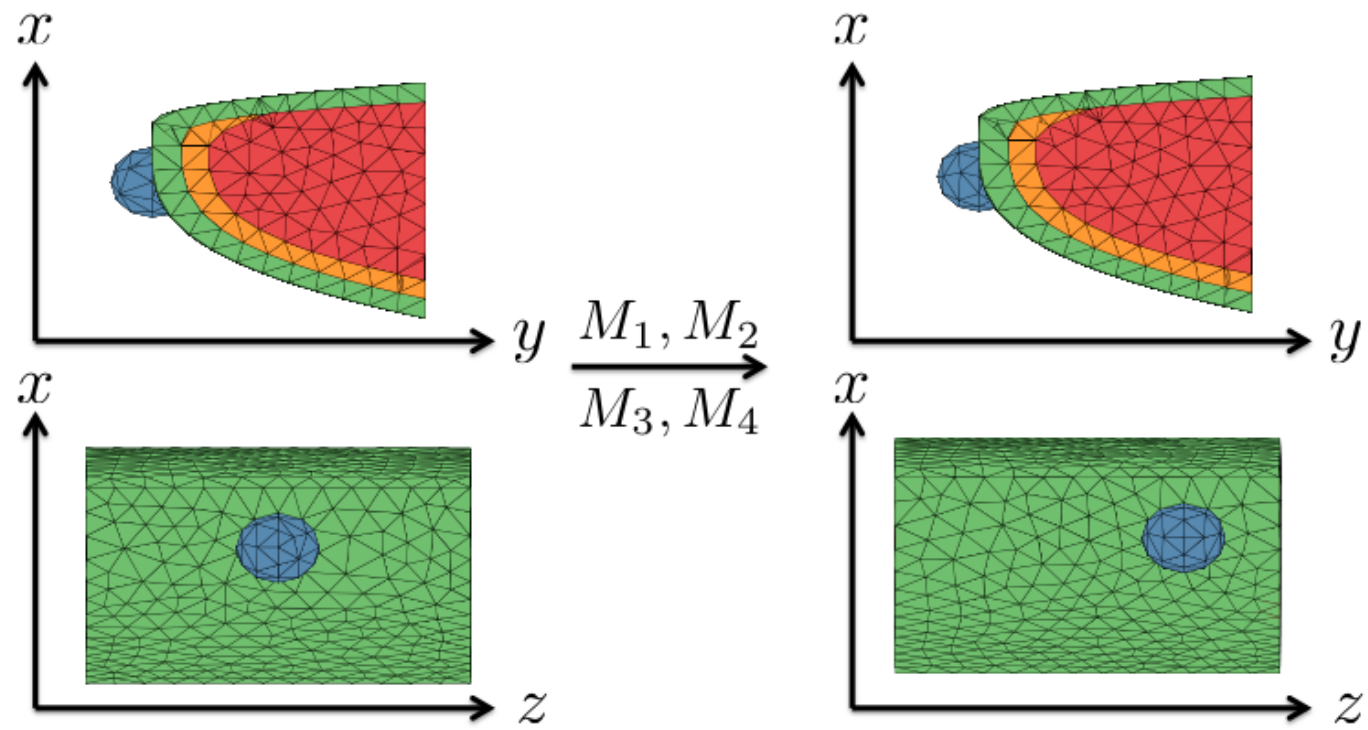

Figure 4.4: Simulation set-up for examination of the interaction of mass and position. The mass and position in the three-dimensional mesh is varied for multiple cases.

The results of this set of simulations shows again that increasing mass causes a decrease in natural frequency. With respect to position, the natural frequency of the vocal fold increases as the polyp moves toward the laryngeal wall ( $x=3.5 \mathrm{~mm})$. These trends are represented in a contour plot of mass vs position for the first mode in Fig.4.5. Higher mode contours are found in Appendix B. As the polyp moves towards the wall, the relative influence of the added mass is reduced due to the damping caused by the fixed boundary. When the polyp is situated directly between the walls $(x=0 \mathrm{~mm})$, the damping effect of the polyp's mass is maximized, resulting in a decrease in natural frequency.

The relative influence of mass and position are illustrated in Fig. 4.6, which displays the changes in natural frequency with respect to polyp mass and position for the first mode. Higher mode influence plots are found in Appendix B. The influence of mass is significantly greater than that of position. The relative influence of stiffness and position appear to be on the same order of magnitude, on the order of $\pm 1 \mathrm{~Hz}$.

The results of this numerical experiment indicate that pitch is minimized if the polyp is centered along the anterior-posterior direction. 
Table 4.4: Mass and position interaction simulation set-up, and first four computed natural frequencies

\begin{tabular}{|l|l|l|l|l|l|l|l|l|l|}
\hline \multirow{2}{*}{ Run } & \multicolumn{4}{|l}{ State } & \multicolumn{4}{l|}{ Natural frequencies (Hz) } \\
\cline { 2 - 10 } & A & B & C & C & $\alpha_{\mathbf{P}}$ & $\omega_{\mathbf{1}}$ & $\omega_{\mathbf{2}}$ & $\omega_{\mathbf{3}}$ & $\omega_{\mathbf{4}}$ \\
\hline 1 & 1 & 1 & 1 & 1 & 2 & 115.34 & 126.29 & 136.13 & 145.26 \\
\hline 2 & 2 & 2 & 2 & 1 & 2 & 113.25 & 124.11 & 127.85 & 144.92 \\
\hline 3 & 3 & 3 & 3 & 1 & 2 & 85.911 & 108.89 & 119.47 & 142.00 \\
\hline 4 & 4 & 4 & 4 & 1 & 2 & 62.031 & 94.300 & 115.32 & 123.12 \\
\hline 5 & 1 & 1 & 1 & 2 & 2 & 115.45 & 126.36 & 136.06 & 145.29 \\
\hline 6 & 2 & 2 & 2 & 2 & 2 & 113.35 & 124.03 & 127.98 & 144.75 \\
\hline 7 & 3 & 3 & 3 & 2 & 2 & 86.078 & 108.48 & 119.42 & 141.81 \\
\hline 8 & 4 & 4 & 4 & 2 & 2 & 62.267 & 93.177 & 114.69 & 125.34 \\
\hline 9 & 1 & 1 & 1 & 3 & 2 & 115.35 & 126.36 & 136.22 & 145.36 \\
\hline 10 & 2 & 2 & 2 & 3 & 2 & 113.93 & 124.41 & 129.93 & 144.82 \\
\hline 11 & 3 & 3 & 3 & 3 & 2 & 90.646 & 109.07 & 119.67 & 142.29 \\
\hline 12 & 4 & 4 & 4 & 3 & 2 & 67.323 & 92.999 & 115.02 & 134.07 \\
\hline 13 & 1 & 1 & 1 & 4 & 2 & 115.44 & 126.27 & 136.30 & 145.26 \\
\hline 14 & 2 & 2 & 2 & 4 & 2 & 114.53 & 124.65 & 132.75 & 144.99 \\
\hline 15 & 3 & 3 & 3 & 4 & 2 & 99.983 & 111.45 & 120.11 & 142.74 \\
\hline 16 & 4 & 4 & 4 & 4 & 2 & 92.834 & 100.96 & 117.09 & 137.51 \\
\hline
\end{tabular}

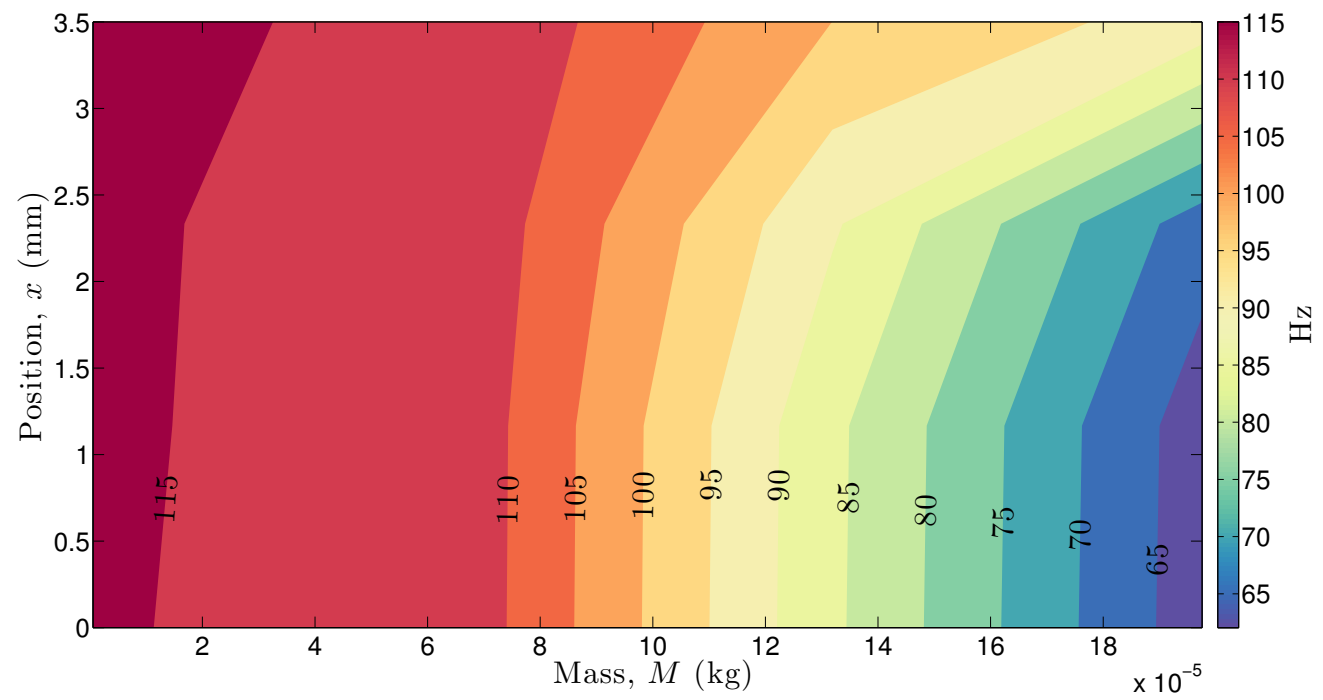

Figure 4.5: Contour plot of fundamental frequency as a function of mass and position. 

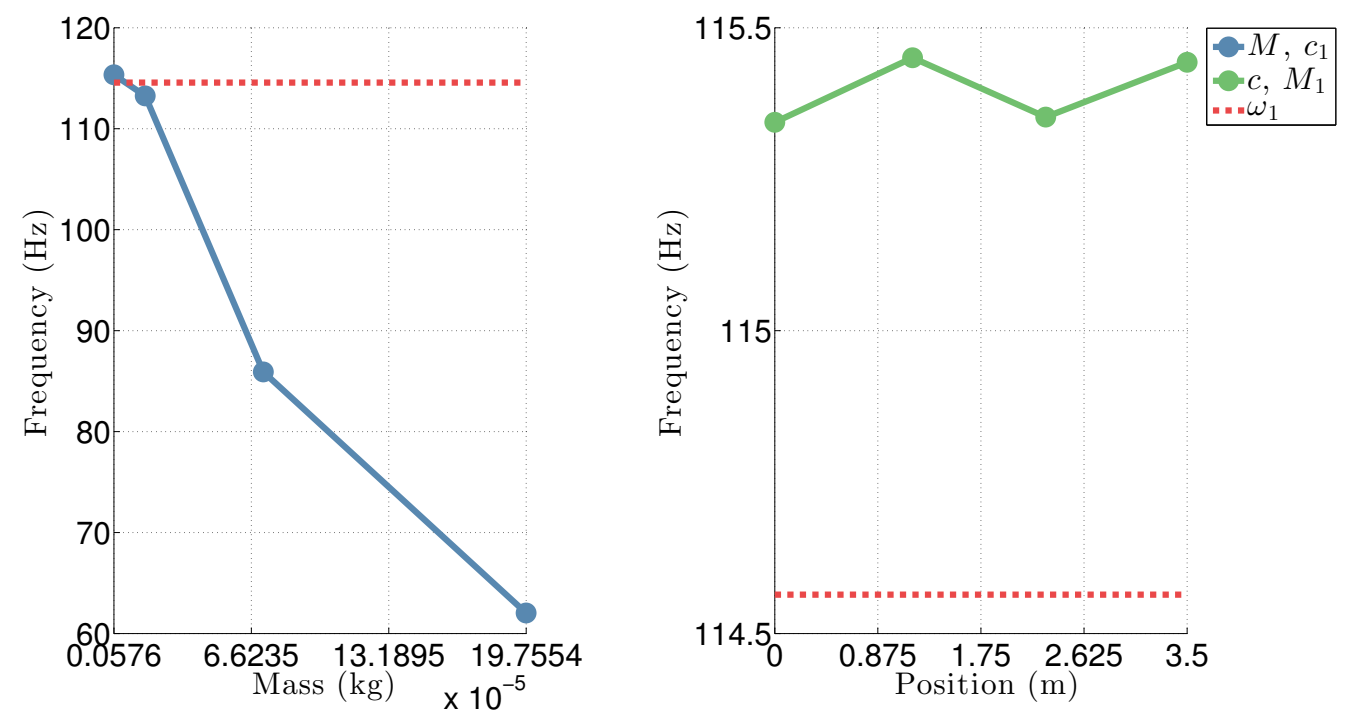

Figure 4.6: Relative influence of mass and position on fundamental frequency. Left: $\omega_{1}$ as a function of mass for $E_{1}$. Right: $\omega_{1}$ as a function of stiffness for $M_{1}$.

\subsubsection{Effects of aspect ratio changes}

To quantify the sensitivity of the minor axis ratios, three sets of three-dimensional simulations were run to examine the effect of varying the ratios $A: B, A: C$, and $B: C$. Recall that axes $A, B$, and $C$ defined the polyp's minor axes in the $z, x$, and $y$ directions, respectively. The position of the polyp was held constant at $x=0 \mathrm{~cm}$, and the stiffness parameter was held constant at $\alpha_{P}=2$. For each ratio, the states of the two relevant minor axes were varied from 1:4 to 4:1, and the remaining axis was set such that the mass of the polyp was constant for all runs.

The states for the set of simulations varying $A: B$ are given in Table 4.5 , along with the computed first four natural frequencies. The visualized variation for each mode is given in Fig. 4.7, which shows that natural frequencies are maximized for the ratios 1:4 and 4:1. This indicates that as mass is distributed over the large medial surface of the vocal fold, the damping effect of the mass is mitigated. In contrast, as mass is concentrated 
Table 4.5: $A: B$ aspect ratio simulation set-up, and first four computed natural frequencies

\begin{tabular}{|l|l|l|l|l|l|l|l|l|}
\hline \multirow{2}{*}{ Run } & \multicolumn{3}{|l|}{ State } & \multirow{2}{*}{ A : B } & \multicolumn{4}{|l|}{ Natural frequencies (Hz) } \\
\cline { 2 - 6 } & $\mathbf{A}$ & $\mathbf{B}$ & $\mathbf{C}$ & & $\omega_{\mathbf{1}}$ & $\omega_{\mathbf{2}}$ & $\omega_{\mathbf{3}}$ & $\omega_{\mathbf{4}}$ \\
\hline 1 & 1 & 1 & 4 & $1: 1$ & 115.50 & 127.02 & 134.76 & 145.16 \\
\hline 2 & 2 & 1 & 3 & $2: 1$ & 114.81 & 126.19 & 131.44 & 144.96 \\
\hline 3 & 3 & 1 & 2 & $3: 1$ & 113.14 & 123.96 & 125.17 & 144.30 \\
\hline 4 & 4 & 1 & 1 & $4: 1$ & 114.62 & 125.52 & 128.04 & 143.39 \\
\hline 5 & 1 & 2 & 3 & $1: 2$ & 114.21 & 124.78 & 131.04 & 145.03 \\
\hline 6 & 1 & 3 & 2 & $1: 3$ & 113.85 & 124.15 & 131.28 & 144.87 \\
\hline 7 & 1 & 4 & 1 & $1: 4$ & 114.76 & 125.53 & 133.70 & 145.36 \\
\hline
\end{tabular}
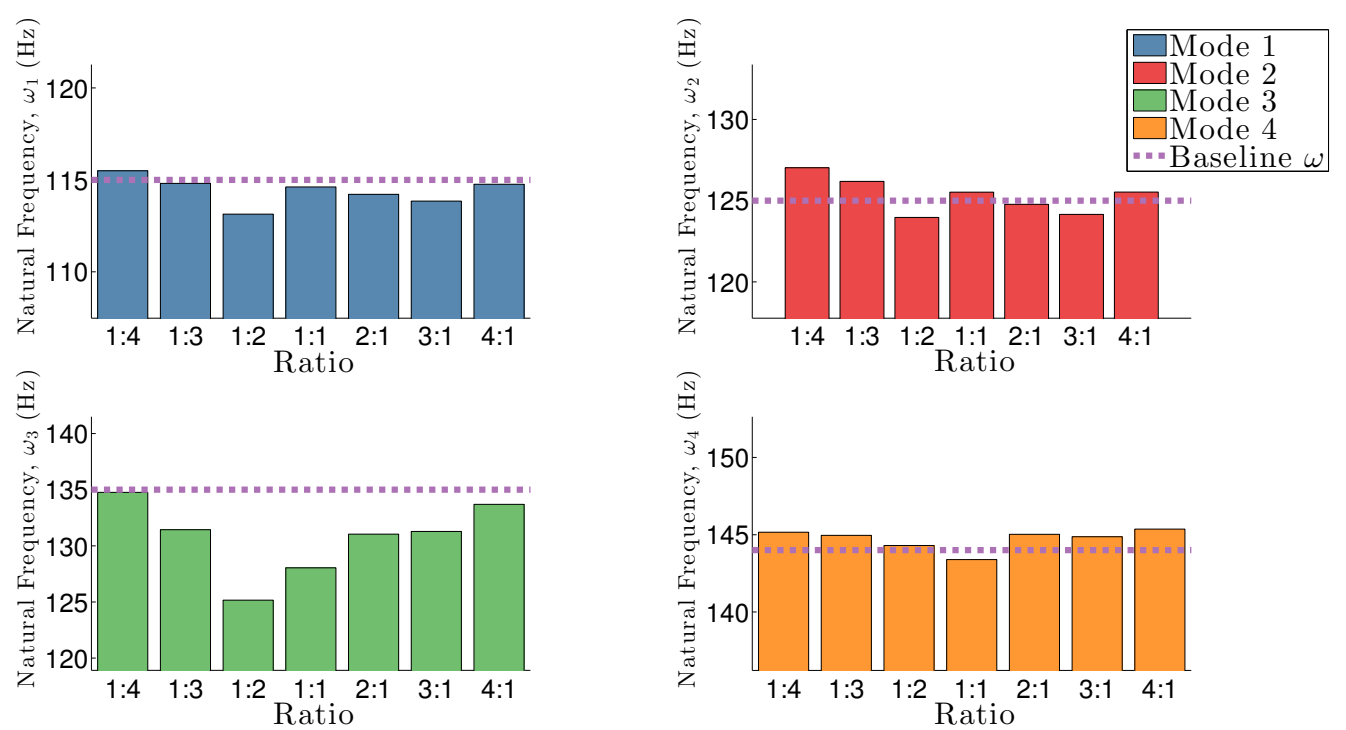

Figure 4.7: Variation in natural frequencies as a function of $A: B$.

(i.e. for a 1:1 ratio), damping is maximized.

Table 4.6 outlines the states for the set of simulations which examine the variation for ratio $A: C$, along with an illustration of its frequency variation in Fig. 4.8, For ratio $A: C$, the fundamental frequency is maximized for the $1: 4$ and 4:1 ratios. Moreover, the 
Table 4.6: $A$ : $C$ aspect ratio simulation set-up, and first four computed natural frequencies

\begin{tabular}{|l|l|l|l|l|l|l|l|l|}
\hline \multirow{2}{*}{ Run } & \multicolumn{3}{|l|}{ State } & \multirow{2}{*}{ A : C } & \multicolumn{4}{|c|}{ Natural frequencies (Hz) } \\
\cline { 2 - 7 } & $\mathbf{A}$ & $\mathbf{B}$ & $\mathbf{C}$ & & $\omega_{\mathbf{1}}$ & $\omega_{\mathbf{2}}$ & $\omega_{\mathbf{3}}$ & $\omega_{\mathbf{4}}$ \\
\hline 1 & 1 & 4 & 1 & $1: 1$ & 114.62 & 125.52 & 128.04 & 143.39 \\
\hline 2 & 2 & 3 & 1 & $2: 1$ & 113.14 & 123.96 & 125.17 & 144.3 \\
\hline 3 & 3 & 2 & 1 & $3: 1$ & 114.81 & 126.19 & 131.44 & 144.96 \\
\hline 4 & 4 & 1 & 1 & $4: 1$ & 115.5 & 127.02 & 134.76 & 145.16 \\
\hline 5 & 1 & 3 & 2 & $1: 2$ & 114.91 & 126.42 & 132.24 & 145.06 \\
\hline 6 & 1 & 2 & 3 & $1: 3$ & 114.19 & 125.53 & 130.96 & 145.17 \\
\hline 7 & 1 & 1 & 4 & $1: 4$ & 114.76 & 125.53 & 133.7 & 145.36 \\
\hline
\end{tabular}
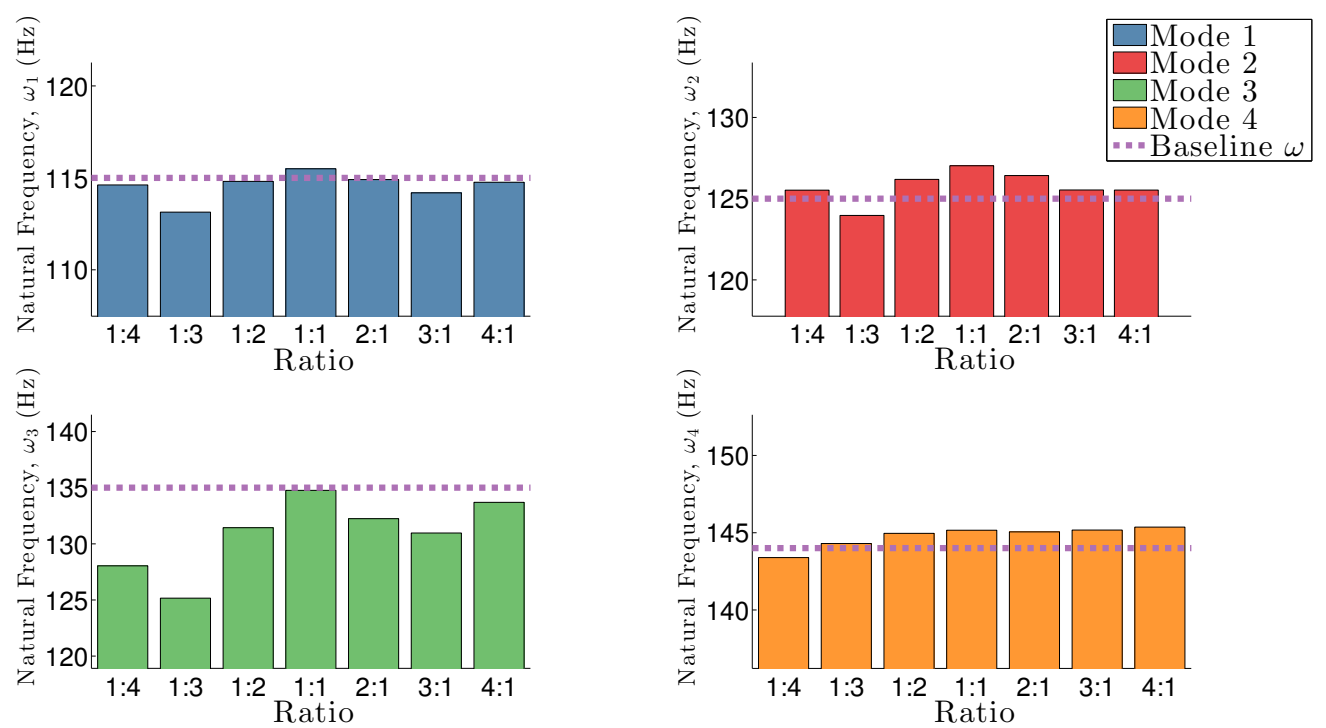

Figure 4.8: Variation in natural frequencies as a function of $A: C$.

influence of this effect increases for the first three modes compared to the results for $A: B$, with a total variation in frequency of $2.3582 \mathrm{~Hz}$ in the first mode, to $9.5945 \mathrm{~Hz}$ in the third mode. This indicates that the elongated polyp in the $A: C$ orientation will not have much effect on the fundamental period of vibration, but rather, on the mucosal wave which travels along the medial surface. 
The influence of minor axis $C$ is on the same order of magnitude as minor axis $B$. However, when comparing the influence of axes $B$ and $C$, the effect of increasing $C$ is larger than corresponding increases in $B$. Thus, axis $C$, in the $y$ direction, is observed to have a greater influence than axis $B$, in the $x$ direction. By increasing $C$, the polyp is extended into the glottis, which has the effect of moving mass away from the fixed boundary conditions, which dampen the vibration. Consequently, the excess mass exhibits a larger effect on damping vibration than if it were extended towards the boundaries, as is the case when increasing $A$ or $B$.

Table 4.7: $B: C$ aspect ratio simulation set-up, and first four computed natural frequencies

\begin{tabular}{|l|l|l|l|l|l|l|l|l|}
\hline \multirow{2}{*}{ Run } & \multicolumn{3}{|l|}{ State } & \multirow{2}{*}{ B : C } & \multicolumn{4}{|l|}{ Natural frequencies (Hz) } \\
\cline { 2 - 7 } & $\mathbf{A}$ & $\mathbf{B}$ & $\mathbf{C}$ & & $\omega_{\mathbf{1}}$ & $\omega_{\mathbf{2}}$ & $\omega_{\mathbf{3}}$ & $\omega_{\mathbf{4}}$ \\
\hline 1 & 4 & 1 & 1 & $1: 1$ & 114.62 & 125.52 & 128.04 & 143.39 \\
\hline 2 & 3 & 2 & 1 & $2: 1$ & 114.21 & 124.78 & 131.04 & 145.03 \\
\hline 3 & 2 & 3 & 1 & $3: 1$ & 113.85 & 124.15 & 131.28 & 144.87 \\
\hline 4 & 1 & 4 & 1 & $4: 1$ & 114.76 & 125.53 & 133.7 & 145.36 \\
\hline 5 & 3 & 1 & 2 & $1: 2$ & 114.19 & 125.53 & 130.96 & 145.17 \\
\hline 6 & 2 & 1 & 3 & $1: 3$ & 114.91 & 126.42 & 132.24 & 145.06 \\
\hline 7 & 1 & 1 & 4 & $1: 4$ & 115.5 & 127.02 & 134.76 & 145.16 \\
\hline
\end{tabular}

Table 4.7 outlines the states for the set of simulations which examine the variation of ratio $B: C$, along with an illustration of its frequency variation in Fig. 4.9, Natural frequencies are maximized for the 4:1 ratio, which again indicates that axis $C$ has a larger influence than axis $B$. Additionally, an increase in natural frequencies is observed for the 1:1 ratio. At this ratio, $B$ and $C$ are minimized, while axis $A$ is elongated. Since $A$ spreads the polyp mass over the medial surface towards the boundaries, the damping effect of this excess mass is counteracted by the damping at the boundaries. Consequently, an 

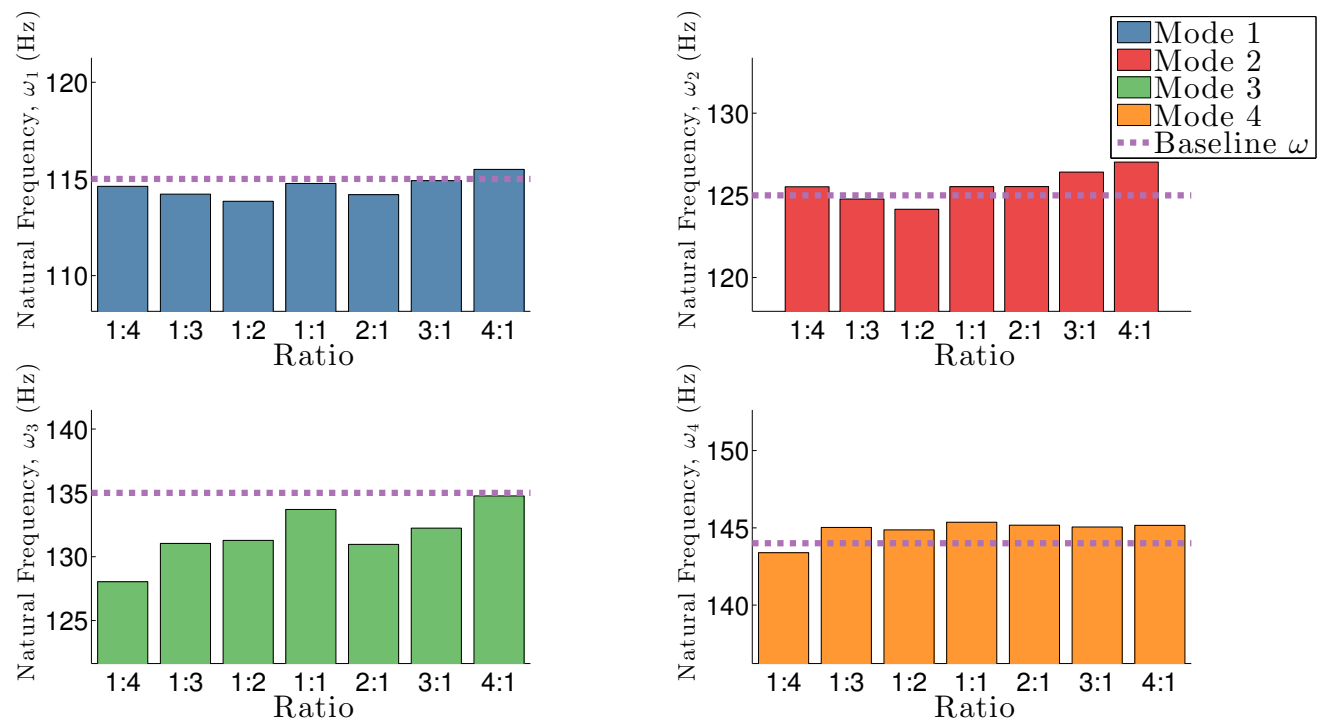

Figure 4.9: Variation in natural frequencies as a function of $B: C$.

increase in natural frequency is observed.

The results of this numerical experiment indicate that elongation of the polyp into the glottis via axis $C$ has a significant damping effect. The spreading of mass over the medial surface with axes $A$ and $B$ results in an increase in natural frequency as the polyp is elongated towards the walls of the larynx.

\subsection{Influence of Polyps on the Pitch-Intensity Depen- dence}

To assess the effect of polyps on pitch-intensity dependence of the vocal folds, the forced vibration of a normal vocal fold is compared to the forced vibration of its paired vocal fold with a polyp. Two sets of simulations are run for the two-dimensional transient case. The set-up for these simulations are illustrated in Fig.4.10, For each pair of vocal folds, vibration is computed for subglottal pressures of $200 \mathrm{~Pa}$ to $1000 \mathrm{~Pa}$ in $100 \mathrm{~Pa}$ increments. These pressures were selected to correspond with physiological values observed 
in phonation. Conversational phonation corresponds with subglottal pressures of 200$300 \mathrm{~Pa}$, and shouting corresponds with subglottal pressure of $1000 \mathrm{~Pa}$ and above [4].
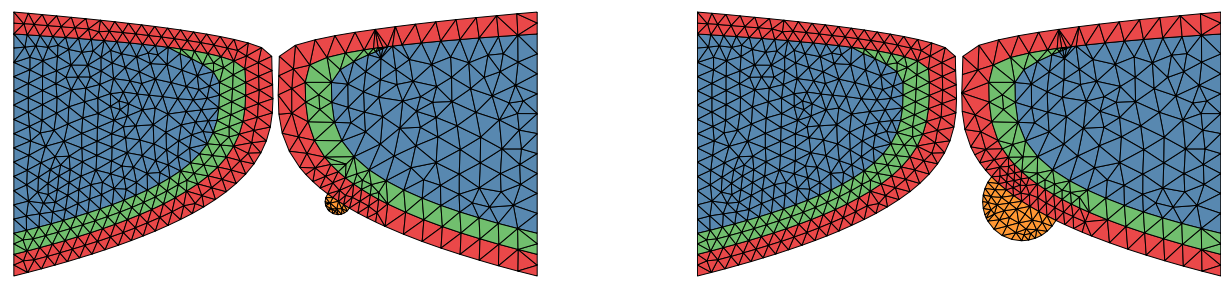

(a) First set of simulations run for polyp of (b) First set of simulations run for polyp of mass $M_{1}$. mass $M_{2}$.

Figure 4.10: Set-up for simulations of forced vibration. Both sets of vocal folds are simulated for subglottal pressures between $200 \mathrm{~Pa}$ to $1000 \mathrm{~Pa}$.

For both sets of simulations, the stiffness parameter is held constant at $\alpha_{P}=2$. In the first set of simulations, as seen in Fig.4.10(a) $A=B=C=1$, which corresponds with mass $M_{1}$, and for the second set of simulations, as seen in Fig. 4.10(b), $A=B=C=2$, which corresponds with a mass $M_{2}$. The initial gap between the vocal folds is $0.02 \mathrm{~cm}$, which corresponds with CT observations from [4]. The vocal fold is moved down the medial surface by $0.25 \mathrm{~cm}$ to avoid contact between the left vocal fold and the polyp. Prior to simulation, a spatial convergence analysis was run, and the meshes for each vocal fold were selected.

Each run monitors the vibration of the node which is set on the flow boundary at the top of the medial surface of the vocal folds. The discrete Fourier transform of this vibration data is then computed using a built-in Matlab function, and the fundamental frequency of vibration is selected from the frequency spectrum. For each vocal fold, a linear trend is fit to determine a relationship between subglottal pressure (intensity) 
and fundamental frequency (pitch). The relationship between these two values is not necessarily linear, however, the metric which will be used to quantify this behaviour will be the slope of this line. In reality, the slope of the trend line is expected to vary for different voice registers. The smallest slope is expected for the high-pitched chest register, $1-3 \mathrm{~Hz} / \mathrm{cm} \mathrm{H}_{2} \mathrm{O}$, and greatest in the falsetto register, 5-10 Hz/cm $\mathrm{H}_{2} \mathrm{O}$ [45].

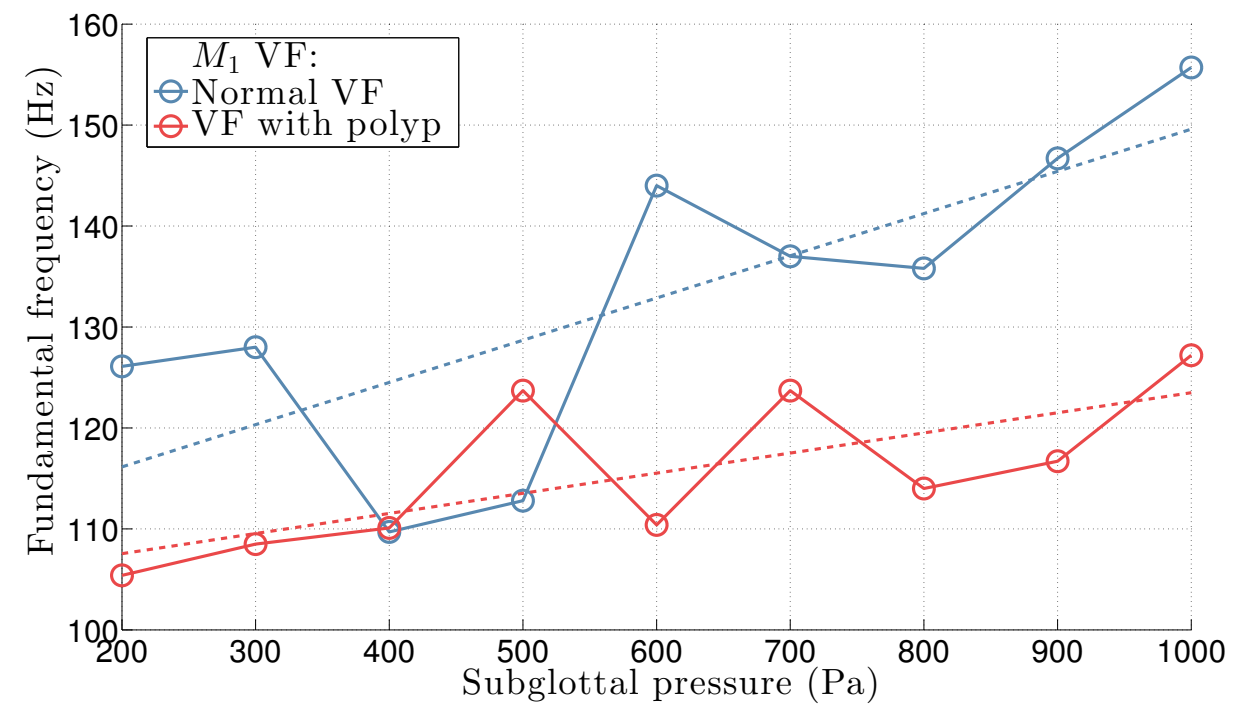

Figure 4.11: Pitch-intensity curves for the vocal fold pairing with a polyp of mass $M_{1}$.

Fig.4.11 illustrates the pitch-intensity curve obtained for the $M_{1}$ vocal fold pairings. The pitch-intensity dependence for the normal vocal fold was found to be $4.1 \mathrm{~Hz} / \mathrm{cm}$ $\mathrm{H}_{2} \mathrm{O}$. This value falls into the physiological range observed in humans, which is 1-10 $\mathrm{Hz} / \mathrm{cm} \mathrm{H}_{2} \mathrm{O}$ [45]. This value dropped for the $M_{1}$ vocal fold to $2.0 \mathrm{~Hz} / \mathrm{cm} \mathrm{H}_{2} \mathrm{O}$. This drop indicates that an individual with this size of polyp would have less range of pitch than a healthy individual.

Fig.4.12 illustrates the pitch-intensity curve obtained for the $M_{2}$ vocal fold pairings. The pitch-intensity dependence was found to be $2.6 \mathrm{~cm} / \mathrm{H}_{2} \mathrm{O}$ for the normal vocal fold, and $2.4 \mathrm{~cm} / \mathrm{H}_{2} \mathrm{O}$ for the vocal fold with the polyp. A drop in the dependence is observed between the vocal folds again. Notably, the disparity between the vocal fold frequencies 
increased, and the normal vocal fold oscillates at a higher frequency when paired with the $M_{2}$ vocal fold. This is likely due to the effective decrease in cross-sectional flow area, as well as the damping effect of the increasing mass of the polyp. For a larger polyp mass, the cross-sectional flow area decreases due to the polyp occupying space in the glottis. This has the effect of pushing the region of flow separation upstream, reducing the aerodynamic loading. Moreover, larger polyp masses dampen vibration of the vocal fold, leading to the pair of vocal folds falling out of phase. It is likely a combination of these two effects which led to this phenomena.

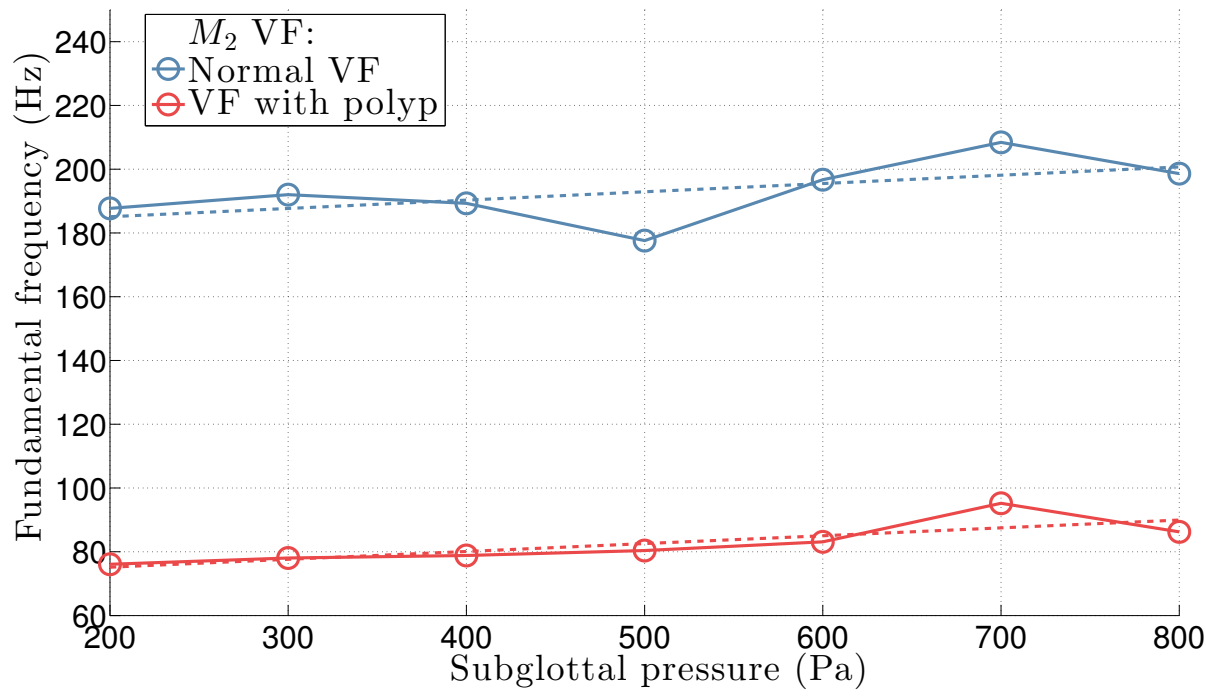

Figure 4.12: Pitch-intensity curves for the vocal fold pairing with a polyp of mass $M_{2}$.

It is reported in scientific literature that the manifestation of vocal fold polyps will lead to disruption in the voice in the form of hoarseness [29], breathiness, and roughness [11]. The results of this numerical experiment support these claims by quantifying the decrease in pitch-intensity dependence. A decrease in pitch would be expected due to the damping of the vocal fold vibration, as pitch is shown to decrease with decreasing abduction length [1, p. 159].

The result of this numerical experiment show that an increase in polyp mass leads to 
a decrease in the pitch-intensity dependence of the vocal fold. 


\section{Chapter 5}

\section{Conclusions and Recommendations}

\subsection{Summary and Contributions}

The present work developed a predictive tool of vocal fold vibration under the influence of a unilateral sessile polyp. Although modeling of vocal fold polyps has been performed [5-7], there is a distinct lack of literature which models the polyp using continuum techniques. The first major contribution of this work is published results and data for the study of a parameterized continuum model of a polyp [43, 44]. As this area of research segues towards the use of continuum techniques for more complex and so-

phisticated studies of surgical procedures [8] and prosthetic design [12, 13], this work may provide a framework from which to build upon.

A transversely isotropic body-cover-ligament model of the vocal folds was implemented. Vibration of the vocal fold was modeled using the finite element method, which solved the equations of motion for a linear stress-strain continuum for a combination of two- and three-dimensional cases. The second major contribution of this work is the development of a continuum based model of a sessile polyp based on its observed properties, and its implementation with the vocal fold model. Polyps were parameterized and simulated on the vocal fold surface as ellipsoids. The parameterization allowed 
control of the polyp's shape, mass, stiffness, and position.

Aerodynamics within the glottis were simulated using a modified form of the Bernoulli equation which accounted for viscous losses and unsteady effects. Transient aerodynamic loading was computed and used as a forcing vector in the finite element structural solver. As a whole, this procedure was implemented as an in-house Fortran 90 code.

The third major contribution of this work is the analysis of the relative influences of polyp properties on vocal fold vibration. The modal structural solver was used to assess the influence of the polyp's parameters on the vibration of the vocal fold. The transient coupled aerodynamic-structural solver was used to assess the effect of polyps on the pitch-intensity dependence of the vocal folds.

This work is intended to build upon the body of voice research which focuses on better understanding the physics which governs phonation, with a view to improving diagnostics of voice disorders and treatment options. Vocal fold polyps may be treated using either voice therapy, surgical removal, or both [15, p. 122]. By quantifying the effects of vocal fold polyps, the selection of an appropriate treatment may be improved, treatment procedures themselves may be improved, and consequently, patient's prognoses may improve.

\subsection{Conclusions}

The implemented model of vocal fold vibration and glottal aerodynamics showed good agreement with experimental and computational data. Natural frequencies and modes of vibrations of the vocal fold model were found to conform with physiological phenomena. The aerodynamic module was shown to sufficiently capture the physics which governs aerodynamic transfer of energy to the vocal folds. 
A polyp's mass was ascertained to be its most influential parameter on vocal fold vibration. Increases in mass were found to lead to a decrease in natural frequency. An increase in a polyp's stiffness was found to increase its natural frequencies. The damping of the polyp's mass was mitigated as the polyp moved toward the anterior or posterior boundaries. The relative influence of stiffness and position were observed to be on the same order of magnitude.

Elongation of the polyp into the glottis was shown to maximize the damping of the excess mass. Spreading of the polyp along the medial surface did not lead to appreciable changes in natural frequency. This phenomena was attributed to the influence of the dampening boundary conditions.

The polyp was found to decrease the pitch-intensity dependence of the vocal fold. Increasing mass of the polyp was found to disrupt the vibration of the vocal fold pair significantly due to the resulting decrease in the effective cross-sectional flow area and vibrational phase shift resulting from damping.

\subsection{Recommendations for Future Work}

Although the results of this study are in good agreement with experimental data, improvement of the methods with regard to their simplifying assumptions can be made.

1. The assumption of a linear constitutive model of stress-strain in the vocal fold, although an effective model, is technically incorrect. The cartilages which are sandwiched between the larynx and the vocal fold, as well as the mucosal tissue which covers the vocal fold, both display time-dependent strain behaviour. Experimental measurements of vocal fold tissue visocelastic properties suggest that exponential models of Young's moduli should be incorporated in the model [73]. Additionally, the strain-dependency of the cartilages at the walls of the vocal folds 
should be modeled. Measurement of these material properties may be performed using traditional engineering techniques, such as stress-strain response with an ergometer [52], and rotational rheometers [54]. Techniques such as stroboscopy may also be incorporated to observe time dependent responses to aerodynamic forcing $|71|$. An implementation of these non-linear equations may improve accuracy, and would represent a novel contribution to the field.

2. Contact between the pair of vocal folds was mitigated through the use of a contact line. Several more sophisticated models of contact exist, and would provide further insight into the flow and vibrational qualities of the larynx.

3. A continuum based implementation of the governing fluid dynamics equations will undoubtedly offer considerable insight into the flow field, and will improve the accuracy of the applied aerodynamic loading. A two-way coupling of the Lagrangian structural system and the Eulerian fluid system may be implemented using an arbitrary Lagrangian-Eulerian framework, and/or a sophisticated meshmoving algorithm.

The parameterization of the polyp presented in this thesis allows for the study of a plethora of other important phenomena, such as the resulting phase shift of the vocal fold pair, flow asymmetry, flow separation locations, and contact stresses.

Another important consideration for future work is the incorporation of realistic meshes of vocal fold geometries and material properties for the purpose of diagnostic investigations. Specifically, there is some interest in the field in making use of CT images of vocal folds [4]. Several methods exist to produce digital renderings from CT data, however, there are some issues which must be resolved before such a technique may be applied in a robust manner. Material properties must be inferred from calibrated images, and the distinction between different materials must be clear for the generation of 
discrete meshes. 


\section{List of References}

[1] W. R. Zemlin, Speech and Hearing Science, Anatomy and Physiology. ERIC, 1968.

[2] F. Alipour, D. A. Berry, and I. R. Titze, "A finite-element model of vocal-fold vibration," The Journal of the Acoustical Society of America, vol. 108, no. 6, pp. 3003-3012, 2000.

[3] M. de Oliveira Rosa, J. C. Pereira, M. Grellet, and A. Alwan, "A contribution to simulating a three-dimensional larynx model using the finite element method," The Journal of the Acoustical Society of America, vol. 114, no. 5, pp. 2893-2905, 2003.

[4] H.Luo, R.Mittal, X.Zheng, S.A.Bielamowicz, R.J.Walsh, and J.K.Hahn, "An immersed-boundary method for flow-structure interaction in biological systems with application to phonation," Journal of Computational Physics, vol. 227, pp. 9303-9332, 2008.

[5] H. D. Jiang J.J., Diaz C.E., "Finite element modeling of vocal fold vibration in normal phonation and hyperfunctional dysphonia: Implications for the pathogenesis of vocal nodules," Annals of Otology, Rhinology, and Laryngology, vol. 107, pp. 603$610,1998$.

[6] Y. Zhang and J. J. Jiang, "Chaotic vibrations of a vocal fold model with a unilateral polyp," The Journal of the Acoustical Society of America, vol. 115, no. 3, pp. 12661269, 2004.

[7] S. Deguchi and Y. Kawahara, "Simulation of human phonation with vocal nodules," American Journal of Computational Mathematics, vol. 1, pp. 189-201, 2011.

[8] K. Ishizaka and N. Isshiki, "Computer simulation of pathological vocal-cord vibration,” The Journal of the Acoustical Society of America, vol. 60, no. 5, pp. 1193-1198, 1976. 
[9] C. A. Rosen, "Complications of phonosurgery: Results of a national survey," The Laryngoscope, vol. 108, no. 11, pp. 1697-1703, 1998.

[10] N. Roy, R. M. Merrill, S. Thibeault, S. D. Gray, and E. M. Smith, "Voice disorders in teachers and the general populationeffects on work performance, attendance, and future career choices," Journal of Speech, Language, and Hearing Research, vol. 47, no. 3, pp. 542-551, 2004.

[11] R. Mittal, B. D. Erath, and M. W. Plesniak, "Fluid dynamics of human phonation and speech," Annual Review of Fluid Mechanics, vol. 45, pp. 437-467, 2013.

[12] N. Isshiki, H. Morita, H. Okamura, and M. Hiramoto, “Thyroplasty as a new phonosurgical technique," Acta oto-laryngologica, vol. 78, no. 1-6, pp. 451-457, 1974.

[13] B. Schneider, W. Bigenzahn, A. End, D.-M. Denk, and W. Klepetko, "External vocal fold medialization in patients with recurrent nerve paralysis following cardiothoracic surgery," European journal of cardio-thoracic surgery, vol. 23, no. 4, pp. 477483, 2003.

[14] H. M. Tucker, J. Wanamaker, M. Trott, and D. Hicks, "Complications of laryngeal framework surgery (phonosurgery),” The Laryngoscope, vol. 103, no. 5, pp. 525-528, 1993.

[15] M. C. Greene, The voice and its disorders. Lippincott, 1980.

[16] B. D. Erath, M. Zañartu, K. C. Stewart, M. W. Plesniak, D. E. Sommer, and S. D. Peterson, "A review of lumped-element models of voiced speech," Speech Communication, vol. 55, no. 5, pp. 667-690, 2013.

[17] J. Jiang, E. Lin, and D. G. Hanson, "Vocal fold physiology," Otolaryngologic Clinics of North America, vol. 33, no. 4, pp. 699-718, 2000.

[18] M. Kob, Physical modeling of the singing voice. Universitätsbibliothek, 2002.

[19] H. Gray, Anatomy of the Human Body. Lea and Febiger, 20 ed., 1918.

[20] S. L. Thomson, L. Mongeau, and S. H. Frankel, "Aerodynamic transfer of energy to the vocal folds," The Journal of the Acoustical Society of America, vol. 118, no. 3, pp. 1689-1700, 2005.

[21] I. R. Titze, "The physics of small-amplitude oscillations of the vocal folds," Acoustical Society of America, vol. 83, no. 5, pp. 1536-1552, 1988. 
[22] A. J. D. Jr., Fundamentals of Aerodynamics. McGraw-Hill Book CompanyHill, 2001.

[23] J. Van den Berg, "Myoelastic-aerodynamic theory of voice production," Journal of speech and hearing research, no. 1, pp. 227-244, 1958.

[24] I. R. Titze and F. Alipour, The myoelastic aerodynamic theory of phonation. National Center for Voice and Speech, 2006.

[25] M. Hirano, "Morphological structure of the vocal cord as a vibrator and its variations," Folia Phoniatrica et Logopaedica, vol. 26, no. 2, pp. 89-94, 1974.

[26] B. H. Story and I. R. Titze, "Voice simulation with a body-cover model of the vocal folds," The Journal of the Acoustical Society of America, vol. 97, no. 2, pp. 1249-1260, 1995.

[27] C. A. Rosen and B. Simpson, Operative techniques in laryngology. Springer Science \& Business Media, 2008.

[28] C. A. Rosen, J. Gartner-Schmidt, B. Hathaway, C. B. Simpson, G. N. Postma, M. Courey, and R. T. Sataloff, "A nomenclature paradigm for benign midmembranous vocal fold lesions," The Laryngoscope, vol. 122, no. 6, pp. 1335-1341, 2012.

[29] B. Erath and M. Plesniak, "Three-dimensional laryngeal flow fields induced by a model vocal fold polyp," International Journal of Heat and Fluid Flow, vol. 35, pp. 93-101, 2012.

[30] K. Ishizaka and J. L. Flanagan, "Synthesis of voiced sounds from a two-mass model of the vocal cords," Bell system technical journal, vol. 51, no. 6, pp. 1233-1268, 1972.

[31] I. Steinecke and H. Herzel, "Bifurcations in an asymmetric vocal-fold model," The Journal of the Acoustical Society of America, vol. 97, no. 3, pp. 1874-1884, 1995.

[32] B. D. Erath, M. Zañartu, S. D. Peterson, and M. W. Plesniak, "Nonlinear vocal fold dynamics resulting from asymmetric fluid loading on a two-mass model of speech," Chaos: An Interdisciplinary Journal of Nonlinear Science, vol. 21, no. 3, p. 033113, 2011.

[33] N. Wan, D. Peng, M. Sun, and D. Zhang, "Nonlinear oscillation of pathological vocal folds during vocalization," Science China Physics, Mechanics and Astronomy, vol. 56, no. 7, pp. 1324-1328, 2013. 
[34] I. R. Titze, "The human vocal cords: A mathematical model part I," Phonetica, vol. 28, no. 3-4, pp. 129-170, 1973.

[35] I. R. Titze, “The human vocal cords: A mathematical model part II,” Phonetica, vol. 29, no. 1-2, pp. 1-21, 1974.

[36] F. Alipour and R. C. Scherer, "Vocal fold bulging effects on phonation using a biophysical computer model," Journal of Voice, vol. 14, no. 4, pp. 470-483, 2000.

[37] D. J. Daily and S. L. Thomson, "Acoustically-coupled flow-induced vibration of a computational vocal fold model," Computers \& structures, vol. 116, pp. 50-58, 2013.

[38] C. Tao, J. J. Jiang, and Y. Zhang, "Simulation of vocal fold impact pressures with a self-oscillating finite-element model," The Journal of the Acoustical Society of America, vol. 119, no. 6, pp. 3987-3994, 2006.

[39] C. Tao and J. J. Jiang, "Mechanical stress during phonation in a self-oscillating finite-element vocal fold model," Journal of biomechanics, vol. 40, no. 10, pp. 21912198, 2007.

[40] C. Tao and J. J. Jiang, "Anterior-posterior biphonation in a finite element model of vocal fold vibration," The Journal of the Acoustical Society of America, vol. 120, no. 3, pp. 1570-1577, 2006.

[41] H. E. Gunter, "A mechanical model of vocal-fold collision with high spatial and temporal resolution," The Journal of the Acoustical Society of America, vol. 113, no. 2, pp. 994-1000, 2003.

[42] L. Wallis, C. Jackson-Menaldi, W. Holland, and A. Giraldo, "Vocal fold nodule vs. vocal fold polyp: answer from surgical pathologist and voice pathologist point of view," Journal of Voice, vol. 18, no. 1, pp. 125-129, 2004.

[43] R. Greiss, J. Rocha, and E. Matida, "Validation of a finite element continuum model of vocal fold vibration," in Acoustics Week in Canada, Canadian Acoustical Association, October 2014.

[44] R. Greiss, J. Rocha, and E. Matida, "Validation of a finite element code for a continuum model of vocal fold vibration under the influence of a sessile polyp," Canadian Acoustics, vol. 43, no. 11, pp. 13-23, 2015.

[45] I. R. Titze, "On the relation between subglottal pressure and fundamental frequency in phonation," Acoustical Society of America, vol. 85, no. 2, pp. 901-906, 1989. 
[46] I. R. Titze and D. T. Talkin, "A theoretical study of the effects of various laryngeal configurations on the acoustics of phonation," Acoustical Society of America, vol. 66, no. 1, pp. 60-74, 1979.

[47] K.-J. Bathe, Finite element procedures. Prentice-Hall Englewood Cliffs, New Jersey, 1996.

[48] D. D. Cook, E. Nauman, and L. Mongeau, "Ranking vocal fold model parameters by their influence on modal frequencies," The Journal of the Acoustical Society of America, vol. 126, no. 4, pp. 2002-2010, 2009.

[49] R. Radovitzky, "Lecture m17: Engineering elastic constants, unified engineering i, ii, iii, \& iv.” Course notes, Spring 2006.

[50] F. Alipour-Haghighi and I. R. Titze, "Viscoelastic modeling of canine vocalis muscle in relaxation," The Journal of the Acoustical Society of America, vol. 78, no. 6, pp. 1939-1943, 1985.

[51] F. Alipour-Haghighi and I. R. Titze, "Elastic models of vocal fold tissues," The Journal of the Acoustical Society of America, vol. 90, no. 3, pp. 1326-1331, 1991.

[52] Y. B. Min, I. R. Titze, and F. Alipour-Haghighi, "Stress-strain response of the human vocal ligament," Annals of Otology, Rhinology, and Laryngology, vol. 104, no. 7, pp. 563-569, 1995.

[53] F. Alipour and I. Titze, "Active and passive characteristics of the canine cricothyroid muscles," Journal of Voice, vol. 13, no. 1, pp. 1-10, 1999.

[54] R. W. Chan and I. R. Titze, "Viscoelastic shear properties of human vocal fold mucosa: measurement methodology and empirical results," The Journal of the Acoustical Society of America, vol. 106, no. 4, pp. 2008-2021, 1999.

[55] D. A. Berry and I. R. Titze, "Normal modes in a continuum model of vocal fold tissues," The Journal of the Acoustical Society of America, vol. 100, no. 5, pp. 3345-3354, 1996.

[56] M. Petyt, Introduction to finite element vibration analysis. Cambridge University Press, 1990.

[57] C. Felippa, “Advanced finite element methods (asen 6367) - spring 2013.” Course notes, May 2013. 
[58] N. M. Newmark, "A method of computation for structural dynamics," Journal of the Engineering Mechanics Division, vol. 85, no. 3, pp. 67-94, 1959.

[59] E. Anderson, Z. Bai, C. Bischof, S. Blackford, J. Demmel, J. Dongarra, J. Du Croz, A. Greenbaum, S. Hammerling, A. McKenney, et al., LAPACK Users' guide, vol. 9. Siam, 1999.

[60] E. A. Carlen, Chapter 4 of Calculus++: The Non-Symmetric Eigenvalue Problem. Georgia Tech Department of Mathematics, 2003.

[61] A. Granados, J. Brunskog, M. K. Misztal, V. Visseq, and K. Erleben, "Finite element modeling of the vocal folds with deformable interface tracking," in Forum Acusticum, 2014.

[62] G. Z. Decker and S. L. Thomson, "Computational simulations of vocal fold vibration: Bernoulli versus navier-stokes,” Journal of Voice, vol. 21, no. 3, pp. 273-284, 2007.

[63] S. M. Han, H. Benaroya, and T. Wei, "Dynamics of transversely vibrating beams using four engineering theories," Journal of sound and vibration, vol. 225, no. 5, pp. 935-988, 1999.

[64] J. M. Gere, Mechanics of Materials. Thomson Brooks/Cole, 6 ed., 2004.

[65] M. Zañartu, L. Mongeau, and G. R. Wodicka, "Influence of acoustic loading on an effective single mass model of the vocal folds," The Journal of the Acoustical Society of America, vol. 121, no. 2, pp. 1119-1129, 2007.

[66] M. Joosten, W. Dettmer, and D. Perić, "Analysis of the block gauss-seidel solution procedure for a strongly coupled model problem with reference to fluid-structure interaction," International Journal for Numerical Methods in Engineering, vol. 78, no. 7, pp. 757-778, 2009.

[67] X. Pelorson, A. Hirschberg, R. Van Hassel, A. Wijnands, and Y. Aurégan, "Theoretical and experimental study of quasisteady-flow separation within the glottis during phonation. application to a modified two-mass model," The Journal of the Acoustical Society of America, vol. 96, no. 6, pp. 3416-3431, 1994.

[68] I. R. Titze, "Regulating glottal airflow in phonation: Application of the maximum power transfer theorem to a low dimensional phonation model," The Journal of the Acoustical Society of America, vol. 111, no. 1, pp. 367-376, 2002. 
[69] J. C. Lucero, “Optimal glottal configuration for ease of phonation," Journal of Voice, vol. 12, no. 2, pp. 151-158, 1998.

[70] C. Felippa, "Introduction to finite element methods (asen 5007) - fall 2015." Course notes, November 2013.

[71] T. Baer, Investigation of Phonation Using Excised Larynxes. PhD thesis, Massachusetts Institute of Technology, February 1975.

[72] P. J. Ross, Taguchi techniques for quality engineering: loss function, orthogonal experiments, parameter and tolerance design. McGraw-Hill Book Company, 1988.

[73] F. Alipour and S. Vigmostad, "Measurement of vocal folds elastic properties for continuum modeling," Journal of Voice, vol. 26, no. 6, pp. 816-821, 2012. 


\section{Appendix A}

\section{Derivation of the Linear Triangular Element}

\section{Matrices}

The objective of this appendix is to derive the element mass and stiffness matrices, $m_{e}$

and $k_{e}$, for the three node triangular element. It was shown in Section 2.4.2 that these matrices were defined by the equations

$$
\left[m_{e}\right]=\int_{V} \rho[N]^{T}[N] d V
$$

and

$$
\left[k_{e}\right]=\int_{V}[B]^{T}[D][B] d V .
$$

This derivation will begin by deriving the shape functions, $[N]$, for the element, and substituting this matrix into Eqn. A.1 and Eqn. A.2.

\section{A.1 Derivation of the Shape Functions}

The triangular element uses its area coordinates to define the shape functions, $[N]$. To demonstrate, we define a point within the element which is not coincident with any 
edge. In Fig. A.1, this point is shown distinct from the black nodes as a yellow circle. The triangle 123 may now be separated into three sub-triangles with their own distinct area. We adopt the convention $A_{i}$ to define the area of the sub-triangle which does not have a vertex at node $i$.

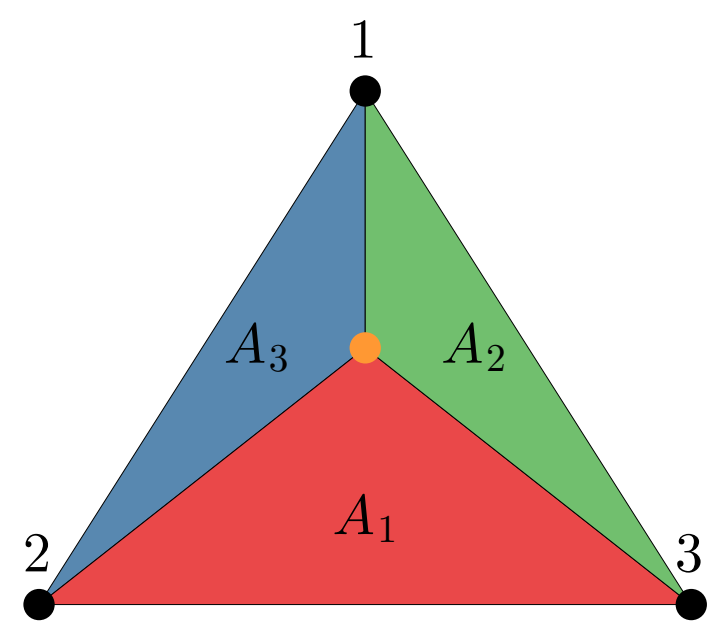

Figure A.1: Sub-triangles defined by an arbitrary point within the element.

We may now express the area of triangle $123, A$, as

$$
\begin{aligned}
& \sum_{i=1}^{3} A_{i}=A \\
\Rightarrow & \sum_{i=1}^{3} \frac{A_{i}}{A}=1 \\
\Longrightarrow & \sum_{i=1}^{3} L_{i}=1
\end{aligned}
$$

where $L_{i}$ are the area coordinates of the triangle [56]. Cartesian and area coordinates are 
related as follows [56]:

$$
\begin{aligned}
& x=x_{1} L_{1}+x_{2} L_{2}+x_{3} L_{3} \\
& y=y_{1} L_{1}+y_{2} L_{2}+y_{3} L_{3}
\end{aligned}
$$

Combining Eqn. A.3 and Eqn. A.4, we develop the following system of equations [56]

$$
\left[\begin{array}{l}
1 \\
x \\
y
\end{array}\right]=\left[\begin{array}{ccc}
1 & 1 & 1 \\
x_{1} & x_{2} & x_{3} \\
y_{1} & y_{2} & y_{3}
\end{array}\right]\left[\begin{array}{c}
L_{1} \\
L_{2} \\
L_{3}
\end{array}\right] .
$$

To obtain an expression for the area coordinates, $L_{i}$, as a function of the nodal coordinates, $\left(x_{i}, y_{i}\right)$, we take the inverse of Eqn. A.5. We adopt the convention

$$
\begin{aligned}
& x_{i j}=x_{i}-x_{j} \\
& y_{i j}=y_{i}-y_{j}
\end{aligned} .
$$

Taking the inverse of Eqn. A.5.

$$
\left[\begin{array}{l}
L_{1} \\
L_{2} \\
L_{3}
\end{array}\right]=\frac{1}{2 A}\left[\begin{array}{ccc}
2 A_{1} & y_{23} & x_{32} \\
2 A_{2} & y_{31} & x_{13} \\
2 A_{3} & y_{12} & x_{21}
\end{array}\right]\left[\begin{array}{l}
1 \\
x \\
y
\end{array}\right] .
$$

we define an expression for the area coordinates as a function of the nodal coordinates, and the coordinates of a point within the domain [70].

Since the area coordinates satisfy the conditions required for shape functions,

$$
N_{i}=L_{i}
$$


Subsequently, we define the displacement functions using the shape functions as interpolation factors,

$$
\begin{aligned}
& u=\sum_{i=1}^{3} N_{i} u_{i} \\
& v=\sum_{i=1}^{3} N_{i} v_{i}
\end{aligned}
$$

where $u$ and $v$ are the displacements in the $x$ and $y$ directions, respectively, and $u_{i}$ and $v_{i}$ are the displacements at node $i[70 \mid$. We re-write this expression

$$
\{\mathbf{q}\}=\left[\begin{array}{l}
u \\
v
\end{array}\right]=[N]\left\{\mathbf{u}_{e}\right\}
$$

where

$$
\left\{\mathbf{u}_{e}\right\}=\left\{\begin{array}{llllll}
u_{1} & v_{1} & u_{2} & v_{2} & u_{3} & v_{3}
\end{array}\right\}^{T}
$$

and

$$
[N]=\left[\begin{array}{cccccc}
L_{1} & 0 & L_{2} & 0 & L_{3} & 0 \\
0 & L_{1} & 0 & L_{2} & 0 & L_{3}
\end{array}\right]
$$

which defines the shape function matrix for the linear triangular element [56, p. 145].

\section{A.2 Deriving the Element Mass Matrix and Stiffness Matri- ces}

Now that $[N]$ has been defined in Eqn. A.12, the element mass matrix defined in Eqn. A.1 may be developed. Typically, this matrix is evaluated using numerical integration, however, in this case, the integral may be solved for the general case [70]. Matrix entry 
$i, j$ in the integrand of Eqn. A.12 are of the form

$$
\left[m_{e}\right]_{i, j}=\rho t \int_{A} L_{k} L_{r} d A \quad \text { for } k=1,2,3 \text {, and } r=1,2,3
$$

where $t$ is the element thickness. This integral may be evaluated using the general integration formula of monomials in triangular coordinates [70]

$$
\left[m_{e}\right]_{i, j}=\rho t \int_{A} L_{1}^{m} L_{2}^{n} L_{3}^{p} d V=\rho \frac{m ! n ! p !}{(m+n+p+2) !} 2 A
$$

which can be simplified to

$$
\left[m_{e}\right]_{i, j}=\rho t L_{k} L_{r}=\left\{\begin{array}{cc}
\frac{\rho t A}{10} & : k=r \\
\frac{\rho t A}{20} & : k \neq r
\end{array} .\right.
$$

The element mass matrix may now be evaluated for each entry using Eqn. A.15. The element stiffness matrix may be evaluated by developing and substituting the element strain matrix, which was given in Section 2.4.2 as

$$
[B]=[\nabla[N], \nabla \times[N]]^{T} .
$$




\section{Appendix B}

\section{Contour and Relative Influence Plots}

This appendix contains contour plots and relative stiffness plots for higher modes of vibration. Although the main body of the thesis focuses on the fundamental mode, it is also important to examine the effects of polyp parameters on the higher modes, as this gives an indication of how the vibration may be altered on the medial surface of the vocal fold during mucosal wave propagation.

\section{B.1 Interaction of mass and stiffness}

The following plots are the contour and relative influence plots for the mass and stiffness numerical experiments, detailed in Section 4.2.2. The general trends observed for the fundamental mode are the same. Mass has a larger influence on frequency than stiffness. Increasing mass decreases frequency. Increasing stiffness increases frequency. 


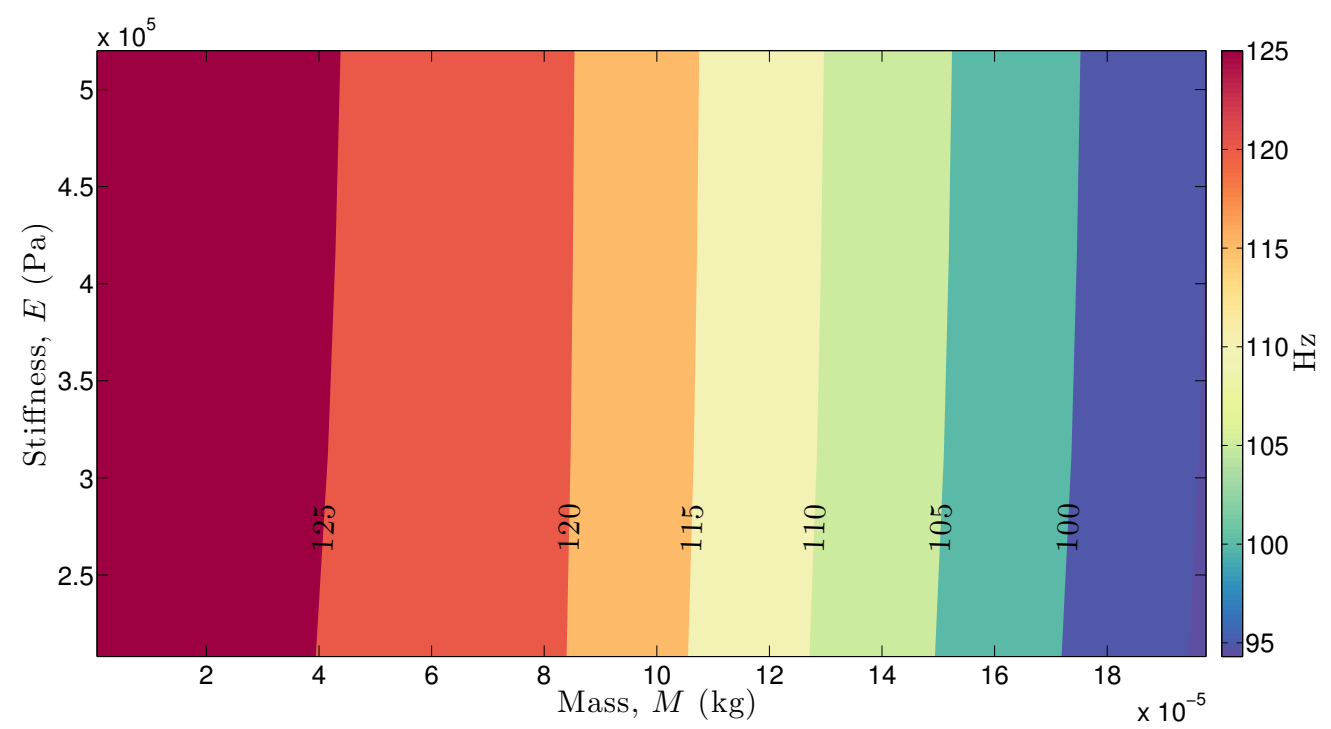

Figure B.1: Contour plot of second natural frequency as a function of mass and stiffness.
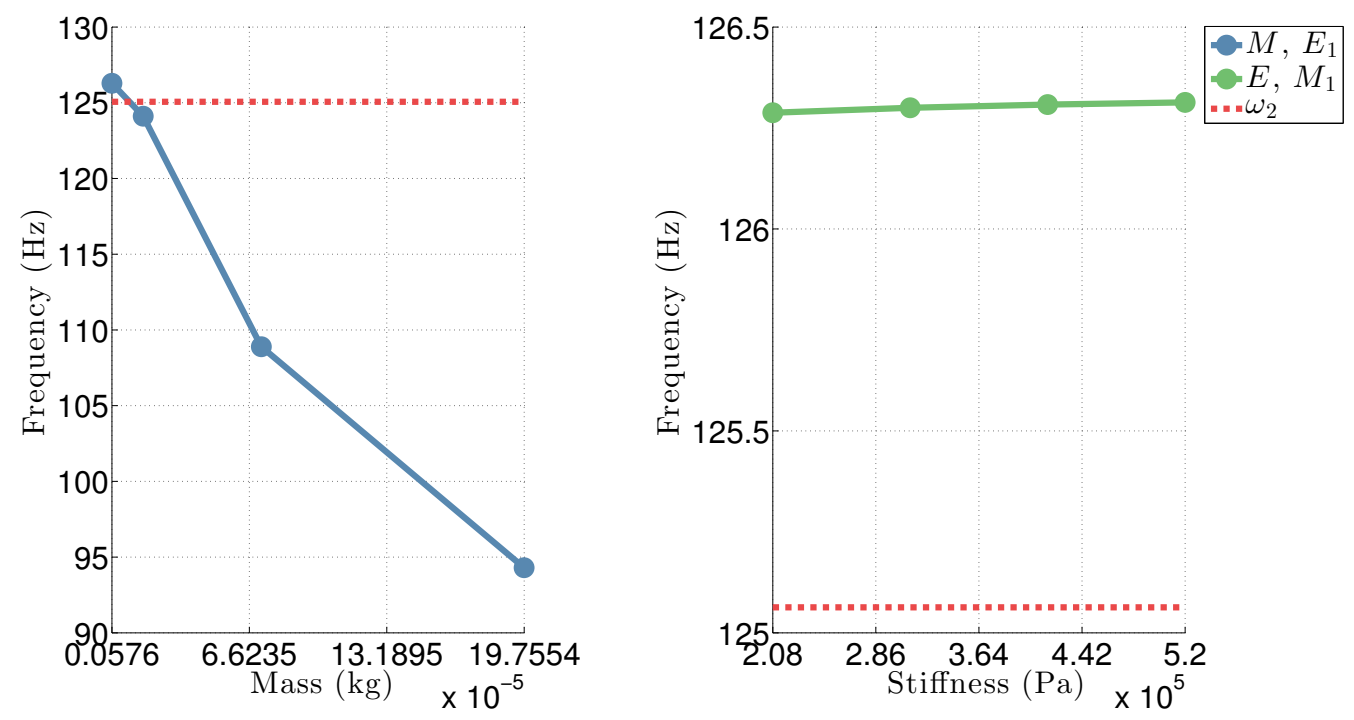

Figure B.2: Relative influence of mass and stiffness on second natural frequency. 


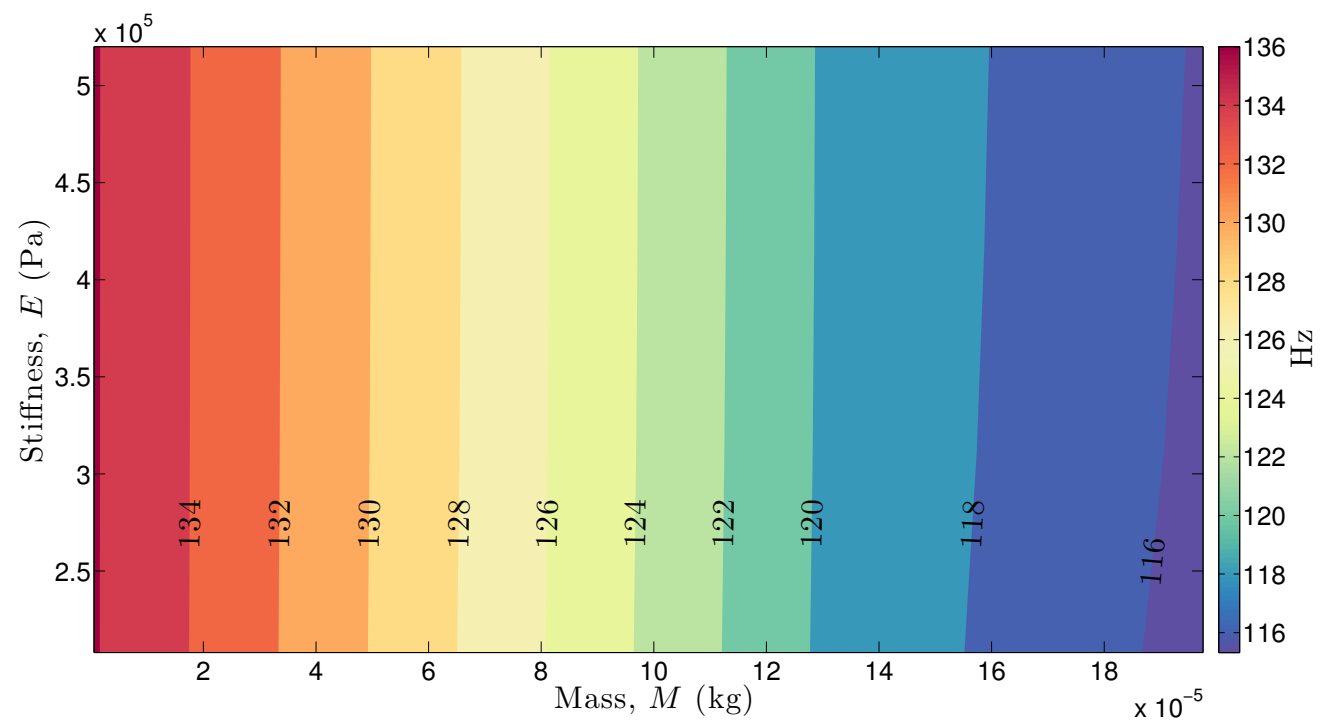

Figure B.3: Contour plot of third natural frequency as a function of mass and stiffness.
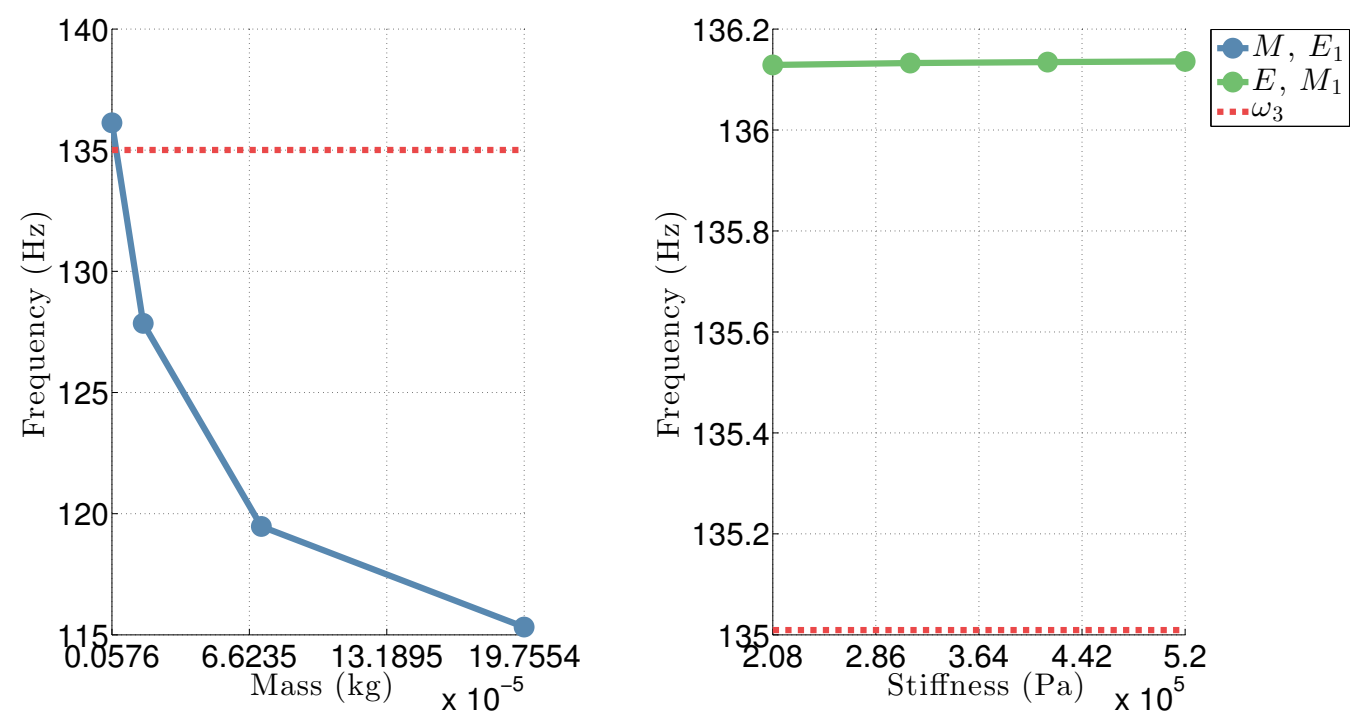

Figure B.4: Relative influence of mass and stiffness on third natural frequency. 


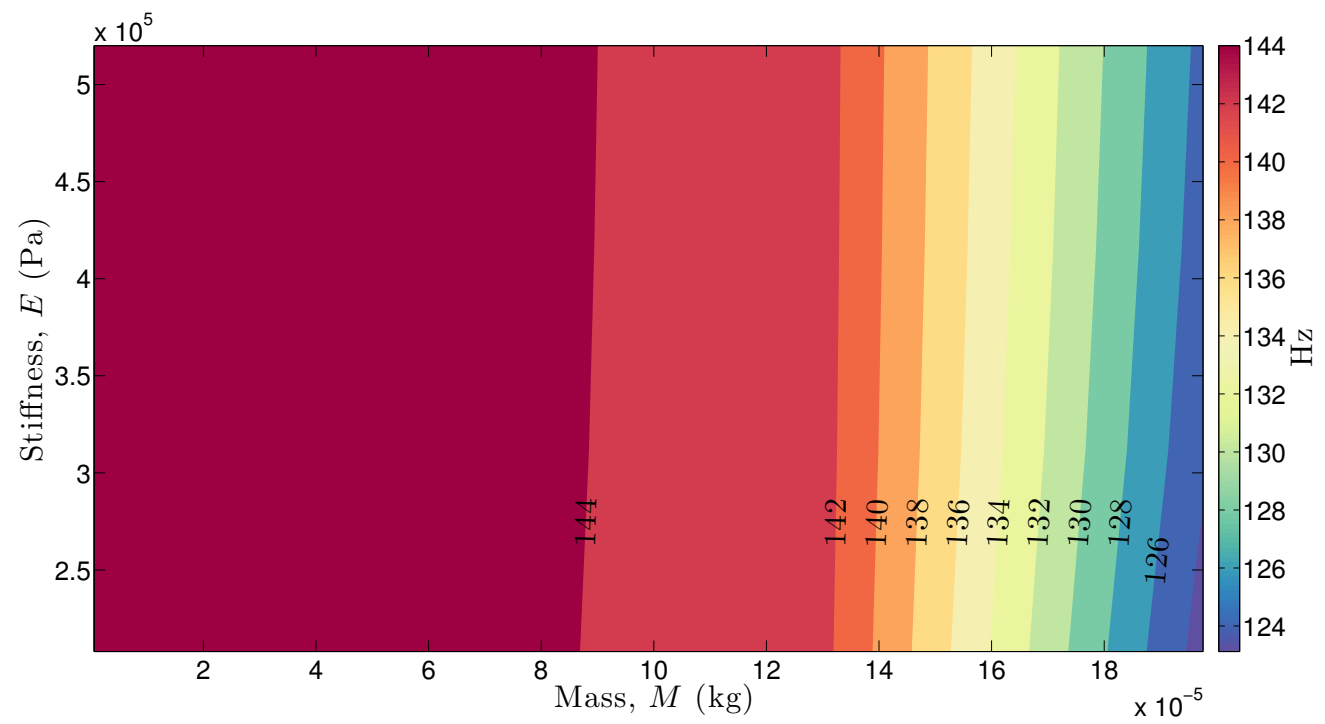

Figure B.5: Contour plot of fourth natural frequency as a function of mass and stiffness.
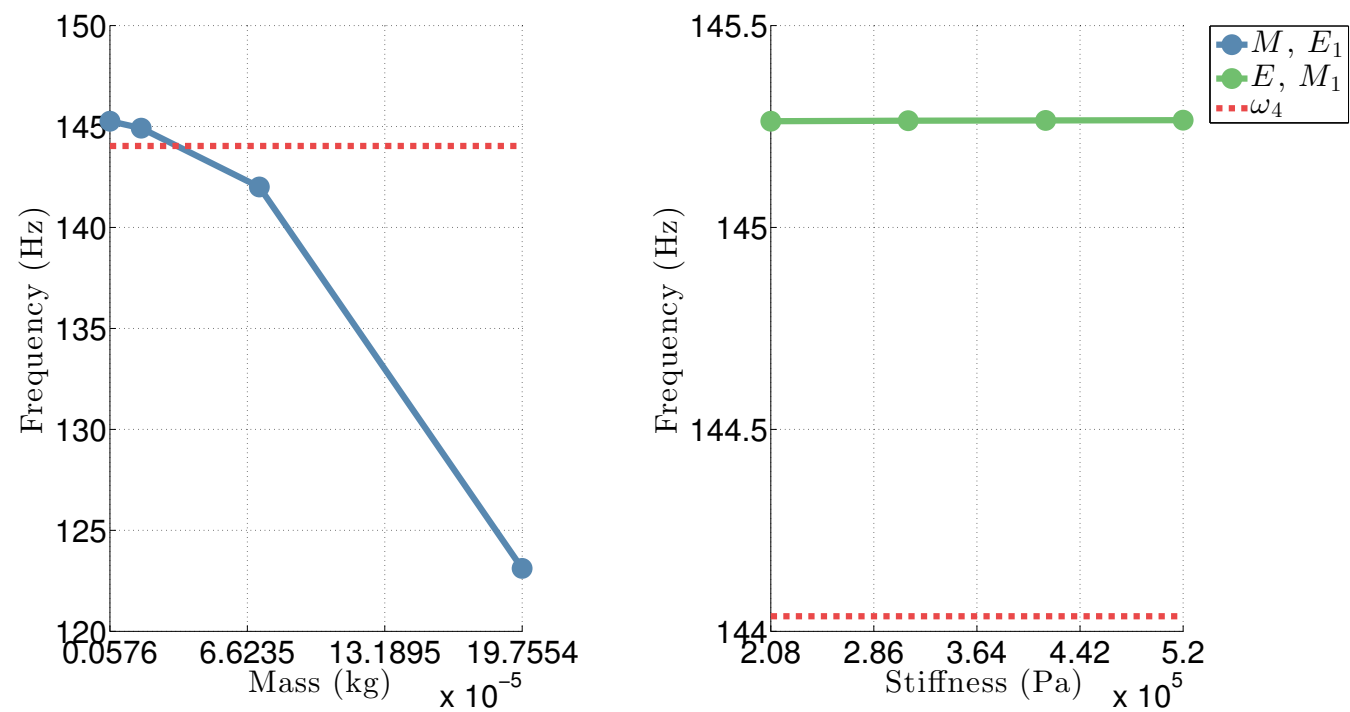

Figure B.6: Relative influence of mass and stiffness on fourth natural frequency.

\section{B.2 Interaction of mass and position}

The following plots are the contour and relative influence plots for the mass and position numerical experiments, detailed in Section 4.2.3. The general trends observed for 
the fundamental mode are the same. Mass has a larger influence on frequency than position. Increasing mass decreases frequency. As the position of the polyp moves towards the walls, frequency increases.

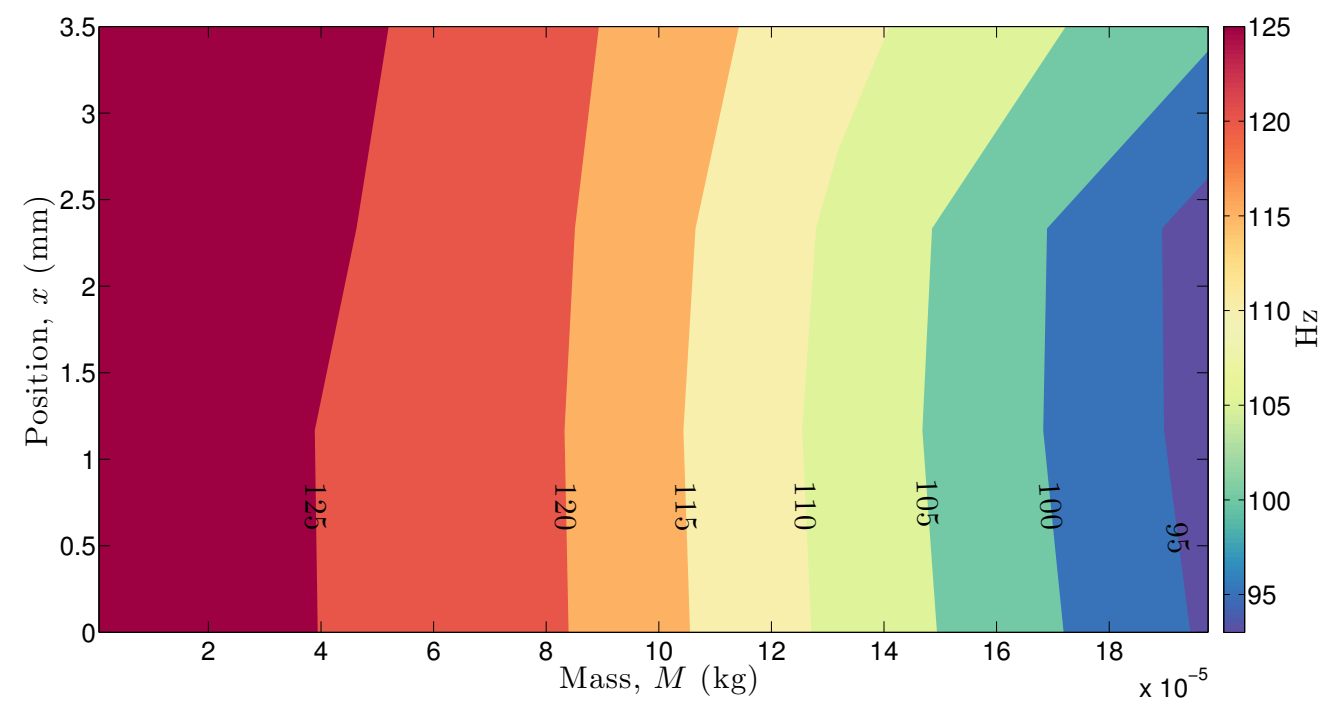

Figure B.7: Contour plot of second natural frequency as a function of mass and position.
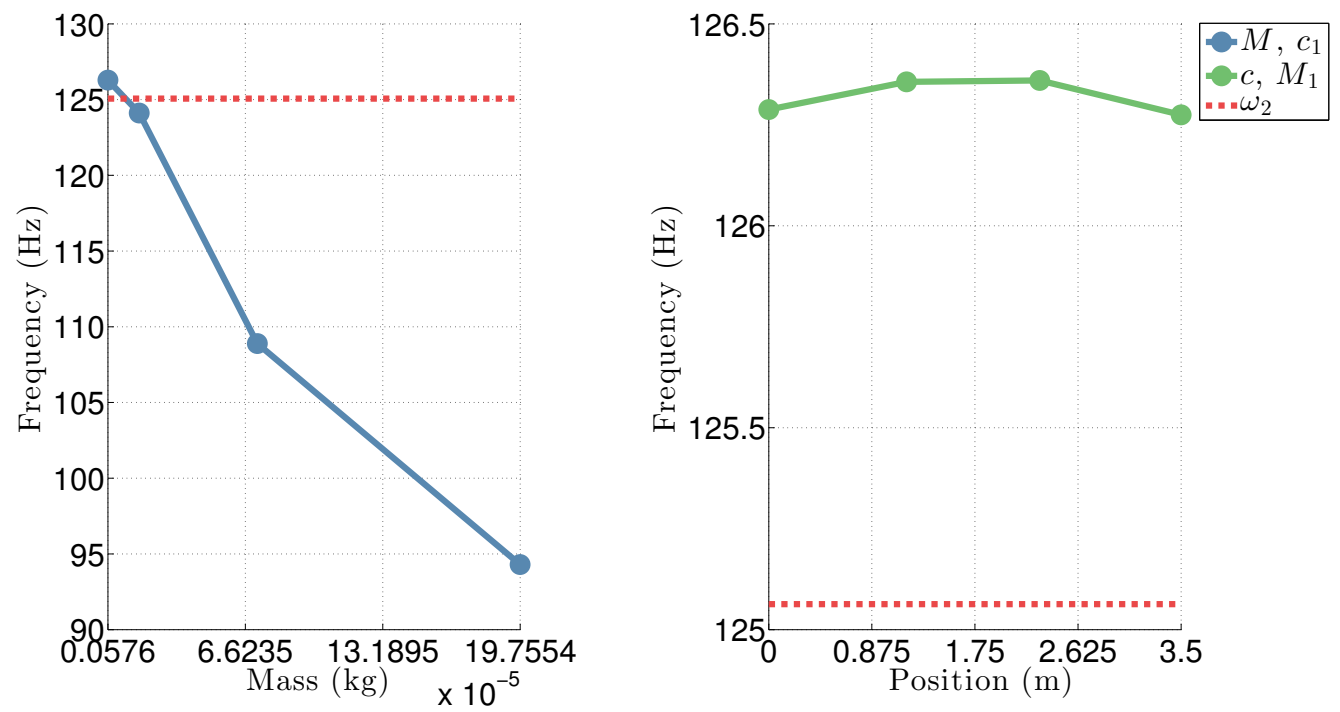

Figure B.8: Relative influence of mass and position on second natural frequency. 


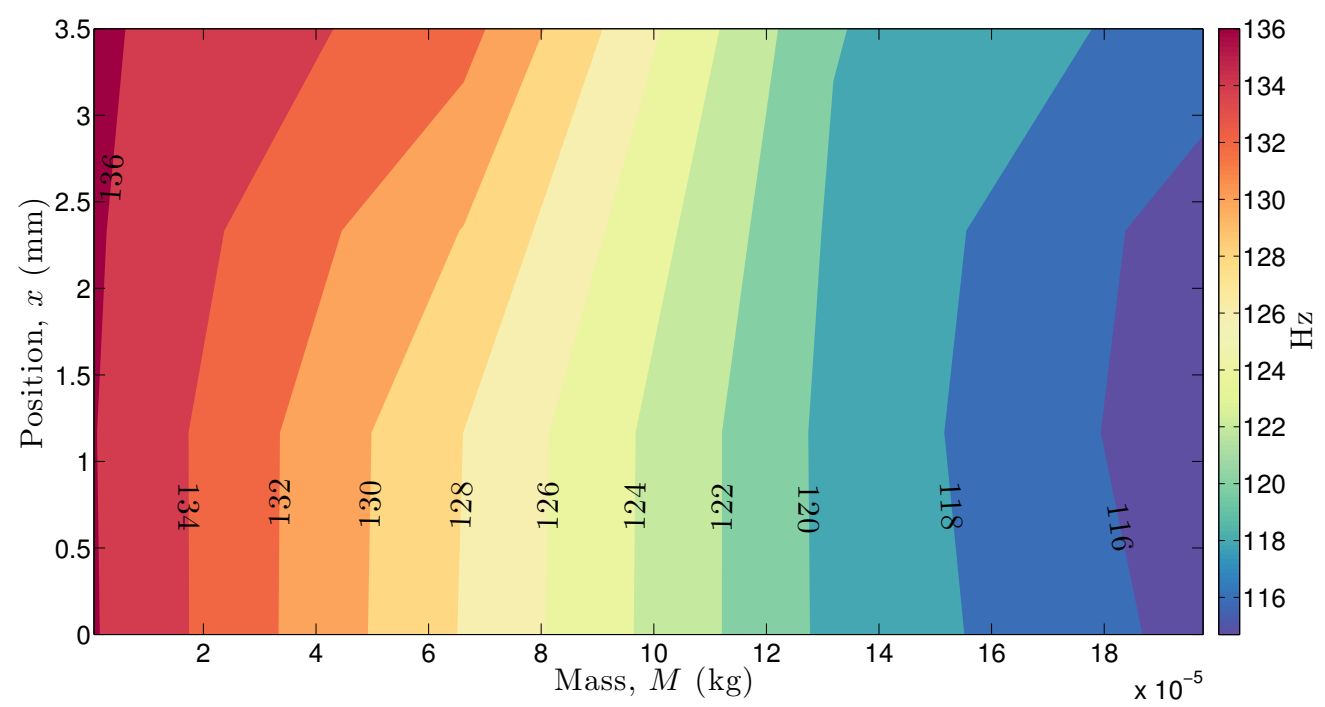

Figure B.9: Contour plot of third natural frequency as a function of mass and position.
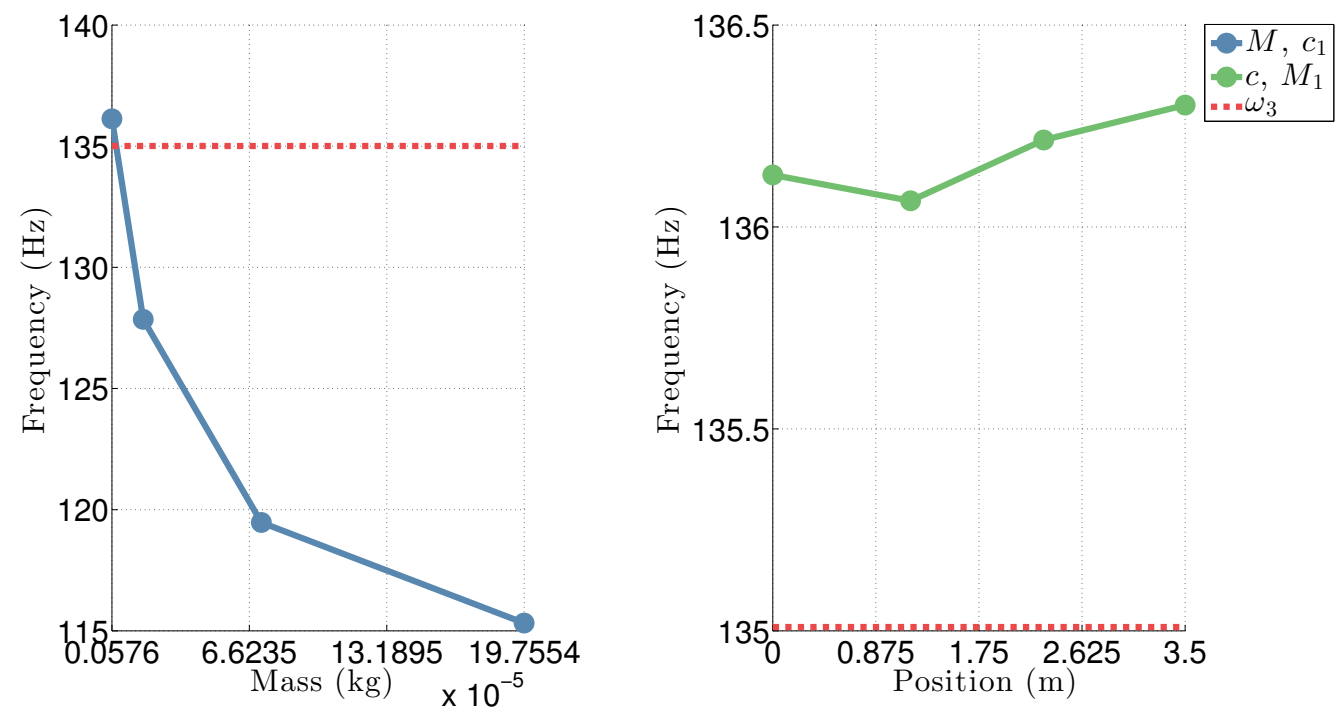

Figure B.10: Relative influence of mass and position on third natural frequency. 


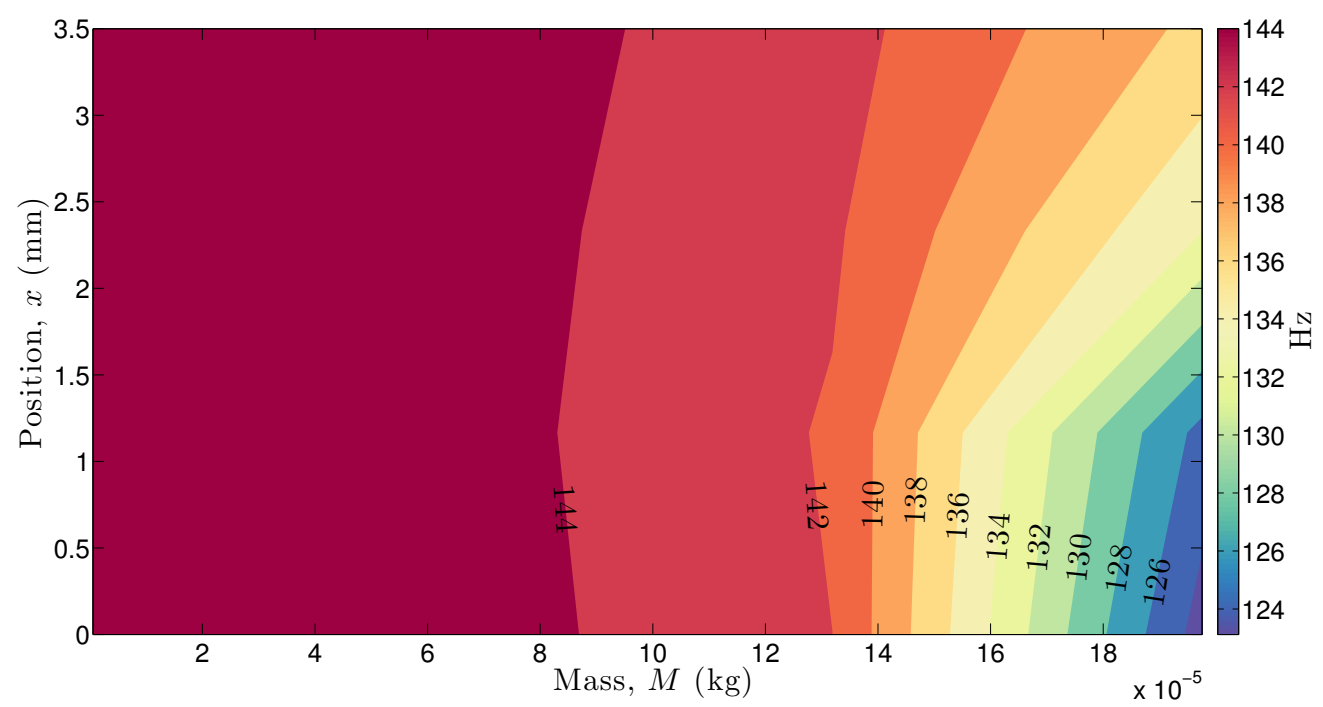

Figure B.1 1: Contour plot of fourth natural frequency as a function of mass and position.
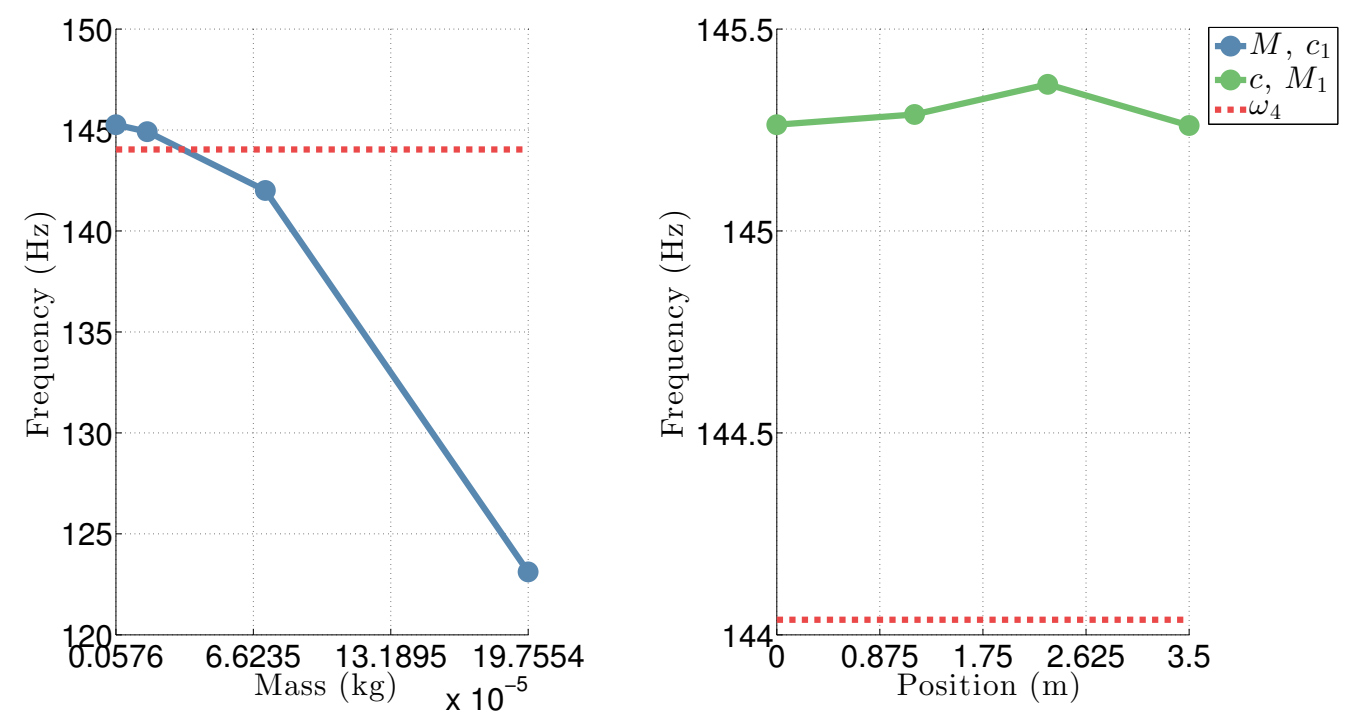

Figure B.12: Relative influence of mass and position on fourth natural frequency. 